\title{
Laser Sintering of Tungsten Nanoparticles for Solar
}

\section{Thermal Receivers}

\author{
A Ph. D. Dissertation
}

Presented to

the Faculty of the School of Engineering and Applied Science

University of Virginia

Doctor of Philosophy (Electrical and Computer Engineering)

by

Ankit Shah

August, 2017 


\title{
Committee Members
}

\author{
Prof. Mool Gupta, Adviser \\ Prof. Andreas Beling, Committee Chair
}

Prof. Nathan Swami

Prof. Joe Campbell

Prof. Stephen McDonnell

Professor Craig Benson, Dean, School of Engineering and Applied Science $-2017$. 


\section{Abstract}

Solar thermal energy is a growing sector in the field of alternative energy. The advantage of solar thermal energy is that in addition to high scalability for utility power plants, it is also able to generate electricity at a later time by making use of thermal storage. Solar thermal technology consists of many components but one of the most important is the solar receiver. The purpose of the receiver is to absorb the concentrated sunlight and retain that heat as fluid carries away the energy that is used by the turbine to generate electricity. The higher the temperature of the fluid, the higher is the efficiency of the thermal conversion system. In order for the receiver to be efficient, it has to be coated with a layer called spectral selective coating. The goal of this coating is to have high solar absorptance and low thermal emittance. Therefore, it should have low reflectance for wavelength $<2 \mu \mathrm{m}$ and high reflectance for $>2 \mu \mathrm{m}$. This will enable absorbed solar energy to be transferred in the form of heat to the fluid with low radiative losses. Spectral selective coatings that are currently used for solar receivers are expensive to manufacture since they require vacuum fabrication techniques. A further drawback is that the current coatings are only stable up to $500-600{ }^{\circ} \mathrm{C}$ in air. The driving factors for long-term performance degradation are oxidation, delamination of coatings, and cracking. Thus there is a need for receiver coatings that have high solar absorptance, low thermal emittance, and can operate at high temperatures in air for tens of years.

In this research, we demonstrate laser sintering of tungsten nano particles to achieve spectral selectivity. Laser sintered tungsten coating have microscale surface roughness that has been used to achieve high solar absorptance and low thermal emittance. Theoretical modeling was carried out to study the interaction of electromagnetic wave with rough surfaces in the visible and infra-red spectrum and in turn determine solar absorptance and thermal emittance. 
Morphological, structural, and compositional properties of laser sintered tungsten films were investigated to understand its optical properties and high temperature stability. We have demonstrated very high solar absorptance $\sim 90.3 \%$ at room temperature and $\sim 87 \%$ after heat treatment at $650{ }^{\circ} \mathrm{C}$. This coating was able to maintain its performance in air when heated at 650 ${ }^{0} \mathrm{C}$ for at least 36 hours making it ideal for solar tower applications. The solar absorptance can be further increased with the use of an anti-reflection film on sintered coatings. 


\section{Acknowledgments}

First of all, I thank my advisor Dr. Mool Gupta for providing me this amazing opportunity to pursue my $\mathrm{Ph}$. D. in collaboration with NASA Langley Research Center. I have faced numerous challenges during my research and his guidance and intuition has been invaluable in helping me overcome them. There were occasions when I could not figure a way to get over some hurdles but having him available to discuss and brainstorm ideas has been a wonderful experience. Dr. Gupta has also been very understanding when it came to personal problems and I deeply appreciate his cooperation for allowing me to overcome them. I sincerely thank him for all of this.

I would also like to thank Dr. Robert Moses from NASA Langley Research Center for providing support for this research and the discussions we have had on developing this technology and its application to NASA. Learning from his experience has helped me understand the bigger picture and how to work in cross-functional teams.

I want to thank Dr. Robert Bryant, Dr. Ravi Shenoy, Dr. Keith Gordon, Mr. James Baughman, Mr. Joel Alexa, Mr. Clay Harold, Mr. Peter Messick, and Mr. David Hartman from NASA Langley Research Center for giving me guidance on the experimental side and helping me with access to characterization equipment and other research tools.

Finally, I would like to thank my grandparents, parents, sister, and my wife for supporting me throughout this journey. 


\section{Contents}

1. Introduction and Literature Review 14

$\begin{array}{ll}\text { 1.1 Solar energy } & 14\end{array}$

$\begin{array}{lr}\text { 1.1.a Photovoltaics } & 14\end{array}$

1.1.b Solar thermophotovltaics $\quad 15$

$\begin{array}{ll}1.1 \text { c. Solar thermal energy } & 16\end{array}$

1.2 Methods for achieving spectral selectivity $\quad 19$

1.2.a Intrinsic absorbers 20

1.2.b Ceramic - metal composite (cermet) 21

$\begin{array}{ll}\text { 1.2.c Surface texturing } & 22\end{array}$

1.3 Laser sintering of metal nanoparticles for spectral selectivity 23

$\begin{array}{ll}\text { 1.3.a Solid state sintering } & 24\end{array}$

$\begin{array}{ll}\text { 1.3.b Liquid phase sintering (LPS) } & 24\end{array}$

$\begin{array}{ll}\text { 1.4 Research objectives and plan } & 27\end{array}$

2. Modeling of Light Absorption and Reflection 30

2.1 Electromagnetic wave interaction with surface roughness 30

$\begin{array}{ll}\text { 2.1.a. Effective medium theory } & 31\end{array}$

2.1.b. Finite difference time domain (FDTD) method 32

2.1.c. Random texture vs. periodic structures $\quad 33$

2.1.d. Effect of anti reflection film 34

3. Experiment: Laser Sintering of Tungsten Nanoparticles 37

$\begin{array}{ll}3.1 \text { Experimental setup } & 37\end{array}$

$\begin{array}{ll}3.2 \text { Tungsten nanoparticles } & 37\end{array}$

$\begin{array}{ll}3.3 \text { Nanoparticle deposition technique } & 39\end{array}$

3.3.a Dip coating $\quad 39$

3.3.b Drop casting in washer $\quad 39$

3.3.c Drop casting $\quad 40$

4. Characterization of Laser Sintered Tungsten Films 4

4.1 Effect of laser process parameters 41

4.1.a Laser power 41

4.1.b Scan speed $\quad 44$

4.2 Morphological study by scanning electron microscope (SEM) 45

4.3 Composition study using energy dispersive x-ray spectroscopy (EDS) $\quad 52$

4.4 Structural characterization using x-ray diffraction (XRD) 55

5. Optical and Infrared Properties of Laser Sintered Tungsten Films 57 
5.1 Optical absorption properties $\quad 57$

5.2 Infrared reflectance properties 58

5.3 Effect of antireflection coating on optical and infrared properties 61

6. Thermal Stability of Laser Sintered Tungsten Films $\quad \mathbf{6 4}$

6.1 Effect of temperature on optical, infrared, and compositional properties $\quad 64$

6.2 Effect of laser sintering environment $\quad 72$

6.2.a Sintering in nitrogen environment $\quad 72$

$\begin{array}{ll}\text { 6.2.b Sintering under atmospheric air } & 73\end{array}$

7. Applications of Laser Sintered Coatings for Solar Thermal and Other $\begin{array}{ll}\text { Areas } & \mathbf{7 5}\end{array}$

$\begin{array}{ll}7.1 \text { Solar tower systems } & 75\end{array}$

$\begin{array}{ll}7.2 \text { Radiative/High emittance coatings } & 77\end{array}$

8. Additional Research $\quad 79$

8.1 Glancing angle deposition (GLAD) of tungsten nanostructures 79

9. Conclusion and Future Work $\quad 82$

9.1 Conclusion $\quad 82$

9.2 Future work $\quad 83$

Bibliography

Appendices

A List of Publications

B Copy of Published Work 


\section{List of Tables}

$\begin{array}{lr}\text { 1. Solar thermal systems } & 19\end{array}$

2. Laser power in Watts 41

3. Solar absorptance for different laser power vs. scan speed obtained by performing only one scan under the laser (' - ' means partial or no sintering) 46

4. EDS data for sintered coating after $1^{\text {st }}$ and $2^{\text {nd }}$ laser scan $\quad 52$

5. Absorptance values at different wavelengths $\quad 57$

6. Solar absorptance of laser sintered coating before and after heat treatment 66

7. EDS data in weight percent (wt\%) before and after heat treatment at $650{ }^{\circ} \mathrm{C} \quad 66$

8. Solar absorptance of sintered coating prepared under nitrogen atmosphere before and after heat treatment at $600{ }^{0} \mathrm{C}$ for 2 hours $\quad 72$

9. Solar absorptance of sintered coating prepared under atmospheric air before and after heat treatment at $600{ }^{0} \mathrm{C}$ for 2 hours $\quad 73$

10. Selective absorber efficiency with solar absorptance of $87 \% \quad 76$ 


\section{List of Figures}

$\begin{array}{ll}\text { 1. Solar cell with charge carriers } & 15\end{array}$

2. STPV system 16

3. Parabolic trough system 17

4. Central tower system 18

5. Solar spectrum and blackbody irradiance at $450{ }^{\circ} \mathrm{C} \quad 20$

6. Bulk material as absorber surfaces $\quad 21$

7. Cermet based spectral selective coating 22

8. Surface texture on surface for increasing solar absorptance (not to scale) 23

9. Microstructural evolution during LPS: (a) Low melting particle is surrounded by high melting particles, (b) low melting point particle starts melting, (c) the melt spreads, and (d) rearrangement of particles in a dense liquid-solid agglomerate $\quad 25$

10. Light trapped by surface texture 30

11. A sub-micron array of cone broken down into layers each having an effective index 31

12. Cone structures on tungsten surface with random spacing and height 34

13. (a) Simulated reflectance for textured tungsten with different average heights.

(b) various distributions of cone periods 35

14. Reflectance for tungsten coated with $\mathrm{TiO}_{2} \mathrm{AR}$ coating 35

15. Experimental setup of laser sintering of tungsten nanoparticles 38

16. Laser sintered coatings with laser power (a) less than $20 \%$, (b) $20-25 \%$, (c) $30-40 \%$, (d) $40-50 \%$, (e) $50-60 \%$, and (f) $>60 \%$

17. SEM images of laser sintered coating at $20 \%$ laser power

18. SEM images sintered coating with $2^{\text {nd }}$ scan having laser power $30 \%$ (a) at 
lower magnification and (b) higher magnification

19. SEM images sintered coating with $2^{\text {nd }}$ scan having laser power $35 \%$ (a) at lower magnification and (b) higher magnification

20. SEM images sintered coating with $2^{\text {nd }}$ scan having laser power $40 \%$ (a) at lower magnification, (b) at higher magnification \& (c) at highest magnification

21. SEM of cross section of sintered layer on stainless steel substrate

22. SEM image of the cross-section of sintered layer on stainless steel substrate showing elemental composition having (a) tungsten, (b) chrome, (c) iron, and (d) nickel

23. XRD spectra of the laser sintered tungsten coating after second scan

24. IR reflectance plot of the laser sintered coating before heat treatment

25. Tungsten 1D periodical grating

26. Normal absorptance plot of the tungsten grating with period $500 \mathrm{~nm}$ and varying heights

27. IR reflectance of (a) SS substrate and (b) sintered coatings before and after $\mathrm{TiO}_{2}$ coating

28. IR reflectance of (a) SS substrate before and after heat treatment at $600{ }^{\circ} \mathrm{C}$ for $2 \mathrm{hrs}$ and (b) tungsten sample before before and after heat treatment at $400{ }^{0} \mathrm{C}$ for $2 \mathrm{hrs}$

29. SEM image of laser sintered tungsten after heat treatment at $650{ }^{\circ} \mathrm{C}$ for two hours

30. (a) IR reflectance of the laser sintered coating before and after heat treatment at $650{ }^{\circ} \mathrm{C}$ and (b) simulated IR reflectance before and after heat treatment at $650{ }^{\circ} \mathrm{C}$ for two hours

31. (a) XRD spectra of laser sintered coating before and after heat treatment at $650{ }^{\circ} \mathrm{C}$ for 
32. IR reflectance plot of laser sintered coating for high radiation applications

33. SEM image of GLAD tungsten structures on SS substrate (provide by ORNL)

34. IR reflectance of GLAD tungsten structures before and after heat treatment 


\section{List of Symbols and Abbreviations}

\begin{tabular}{|c|c|}
\hline$\alpha$ & Solar absorptance \\
\hline$\varepsilon$ & Thermal emittance \\
\hline$\mu \mathrm{m}$ & micrometer \\
\hline$\lambda$ & Wavelength \\
\hline $\mathrm{f}$ & Fill factor \\
\hline$\gamma$ & Material absorption coefficient \\
\hline $\mathrm{z}$ & Depth \\
\hline $\mathrm{Al}_{2} \mathrm{O}_{3}$ & Aluminum oxide \\
\hline $\mathrm{CO}_{2}$ & Carbon dioxide \\
\hline $\mathrm{Cr}-\mathrm{Cr}_{2} \mathrm{O}_{3}$ & Crome chromium oxide \\
\hline $\mathrm{Ni}-\mathrm{Al}_{2} \mathrm{O}_{3}$ & Nickel aluminum oxide \\
\hline $\mathrm{SiO}_{2}$ & Silicon dioxide \\
\hline $\mathrm{TiO}_{2}$ & Titanium dioxide \\
\hline $\mathrm{Ni}-\mathrm{Al}_{2} \mathrm{O}_{3}$ & Nickel aluminum oxide \\
\hline Nd: YAG & Neodymium-doped yttrium aluminium garnet \\
\hline $\mathrm{Si}_{3} \mathrm{~N}_{4}$ & Silicon nitride \\
\hline $\mathrm{W}-\mathrm{Al}_{2} \mathrm{O}_{3}$ & Tungsten aluminum oxide \\
\hline AR & Anti reflection coating \\
\hline $\mathrm{BCC}$ & Body centered cubic \\
\hline EMT & Effective medium theory \\
\hline EDS & Energy dispersive spectroscopy \\
\hline FDTD & Finite difference time domain \\
\hline
\end{tabular}




$\begin{array}{ll}\text { HTF } & \text { Heat transfer fluid } \\ \text { IR } & \text { Infrared } \\ \mathrm{I}_{0} & \text { Intensity } \\ \text { LPS } & \text { Intensity at surface } \\ \text { MEEP } & \text { Liquid phase sintering } \\ \text { SS } & \text { MIT electromagnetic equation propagation package } \\ \text { SSS } & \text { Stainless steel } \\ \text { SEM } & \text { Solid state sintering } \\ \text { TMM } & \text { Scanning electron microscope } \\ \text { UV } & \text { Transfer matrix method } \\ \text { wt } \% & \text { Ultraviolet } \\ \text { XRD } & \text { Weight percent } \\ \text { W } & \text { W-ray diffraction } \\ \end{array}$




\section{Chapter 1. Introduction and literature review}

\subsection{Solar Energy}

Solar energy can be utilized in multiple ways to produce heat and electricity. There are three ways by which solar light can be converted: photovoltaics (PV), solar thermophotovoltaics (STPV), and solar thermal.

a. Photovoltaics: The photovoltaic effect is the phenomenon by which light directly gets converted into electricity. When light of relatively low energy is absorbed by a semiconductor, usually silicon, heat is generated without altering the electrical properties of the material. This causes the atoms of silicon to vibrate but not break loose. The electrons soon return to their original lower energy levels giving off heat as the energy they had gained. Light with higher frequency which in turn corresponds to having greater energy can alter the electrical properties of the crystal. If such wavelength of light strikes a bound electron, the electron is separated from its place in the crystal. This leaves behind a silicon bond missing an electron and frees an electron to move about in the crystal. A bond missing an electron is called a hole. This electron is in the crystal's conduction band because free electrons are the means by which electricity flows. A hole in a silicon crystal can, like a free electron, move about the crystal. An electron from a bond near a hole can easily jump into the hole, leaving behind an incomplete bond, i.e., a new hole. This happens fast and frequently and electrons from nearby bonds trade positions with holes, sending holes randomly and erratically throughout the solid. Both the conduction-band electrons and the holes play important part in the electrical behavior of PV cells. A PV cell contains an electric field that is set up by opposite electric charges. The field generated makes the electrons flow to one side of the cell and holes to the other. This charge separation sets up a voltage 
difference between the cell which is used to drive an electric current in an external circuit.

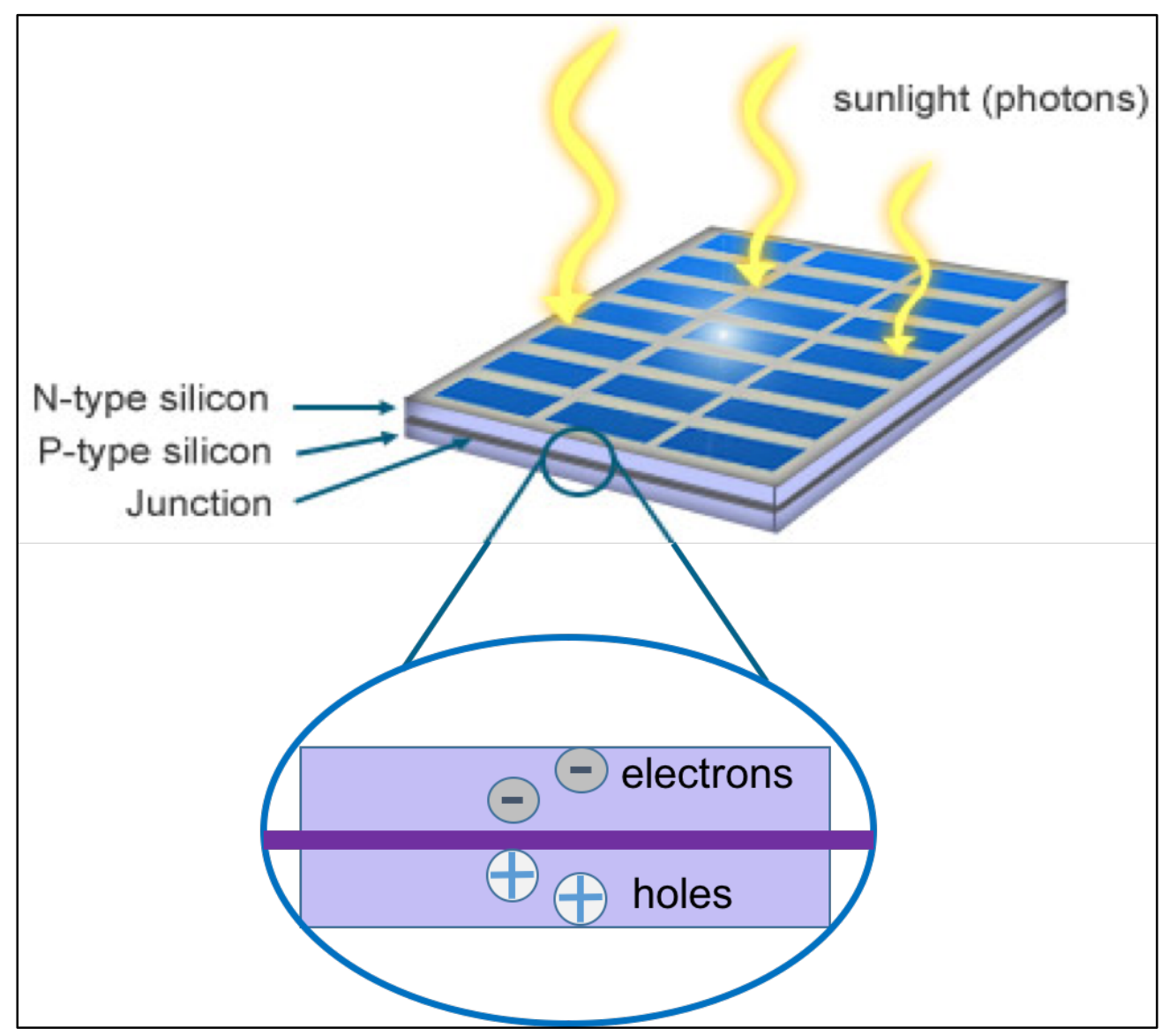

Fig 1. Solar cell with charge carriers [1]

b. Solar thermophotovoltaic (STPV): STPV systems can provide higher solar conversion efficiencies than only using solar cells. This is achieved by using an intermediary material to modify the spectrum of incoming solar radiation to be better matched to a PV cell. To accomplish this, incoming concentrated solar radiation is absorbed by the absorbing surface. As the temperature of the absorber rises, it emits large amounts of thermal radiation out of the emitting surface also called the emitter. This radiation can be absorbed by a PV cell to 
create electricity [2]. Since PV cells are highly efficient for only certain band of the spectrum, the emitting surface can be modified to create a narrow-band thermal emission. This narrow band can greatly improve the efficiency of PV devices as they have been matched to the absorption spectrum of the PV cell. Since now the matching is not limited to only solar spectrum, the overall STPV system can utilize the full solar spectrum and use all the heat to emit only in the necessary band allowing it to exceed the efficiencies of PV systems. STPV system is comprised of the following parts: an absorbing, an emitting surface, and a PV cell. Even though STPV system can be more efficient that only a solar cell system, lack of heat storing capability and a more complex design makes solar thermal a better option.

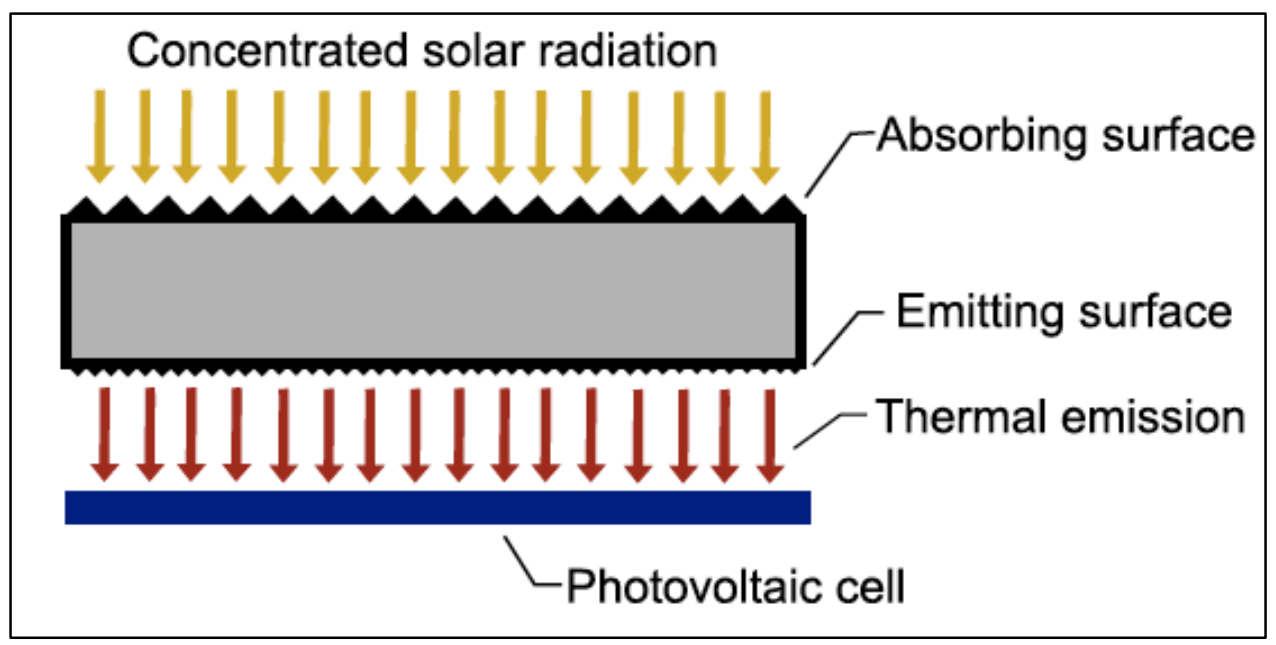

Fig 2. STPV system [2].

c. Solar thermal energy: Concentrated solar power (CSP) is a promising field in renewable energy. CSP systems use mirrors to concentrate sunlight onto a receiver that transfers the generated heat to a heat transfer fluid (HTF). The hot fluid is then sent to a heat exchanger 
where it boils water to run a steam turbine and generate electricity. CSP systems need high photothermal conversion efficiency of the receiver in order to increase the overall efficiency of the system. For efficient energy conversion, high operating temperatures, $>650{ }^{0} \mathrm{C}$, are desired which in turn require highly efficient receiver surfaces at those temperatures. Figure 3 below shows a parabolic trough system where sunlight is collected by large array of parabolic mirrors with the receiver tube running along its axis. The receiver tubes are placed at the focal point of the parabola so that the sunlight is concentrated by parabolic mirrors onto the receiver tubes. Usually these parabolic troughs are arranged in the north-south direction so that they can track the sun from east to west through out the day for maximum efficiency. The concentration ratio for parabolic trough systems is usually between 30 and $100[3]$.

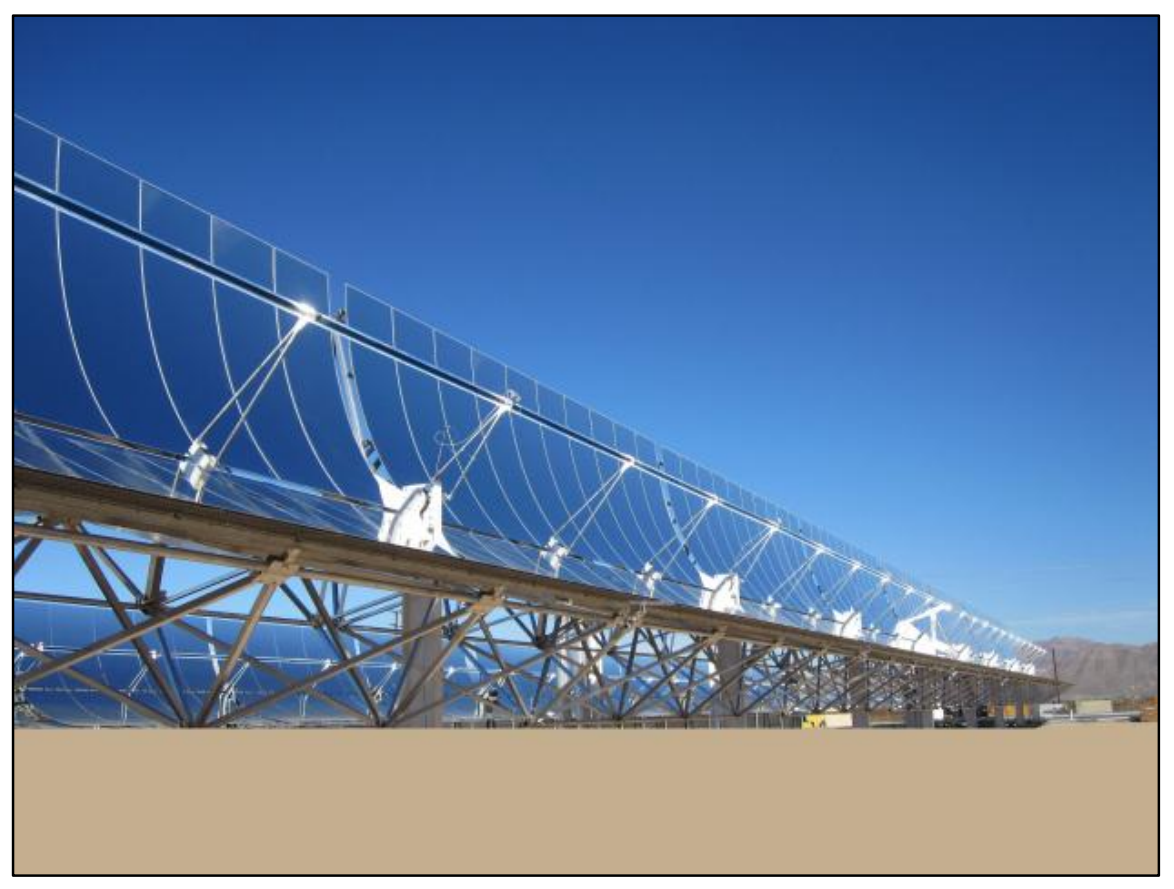

Fig 3. Parabolic trough system [4] 


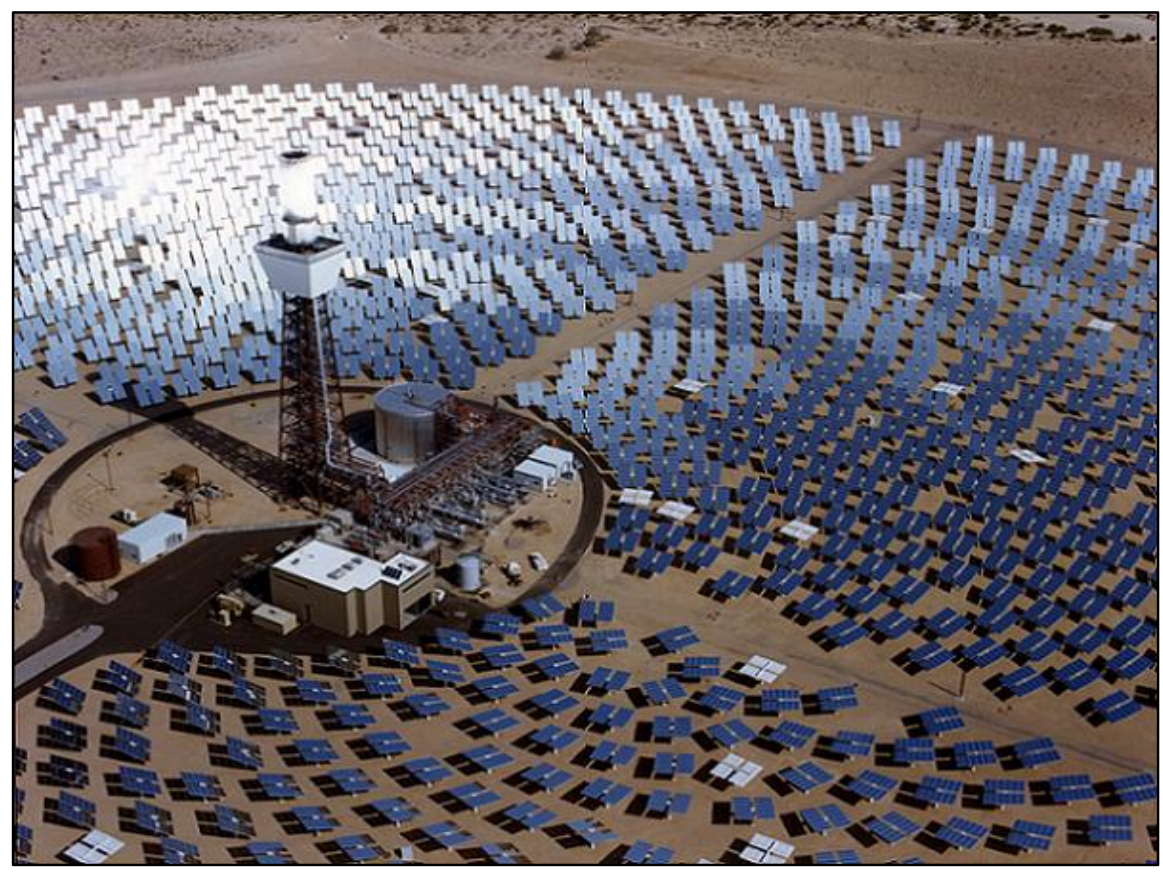

Fig 4. Central tower system [5]

Figure 4 shows a central tower system where mirrors on the ground concentrate sunlight onto a central receiver on top of the tower. There are several mirrors called heliostats lying around a central tower with receiver on the top. Heliostats are capable of having a concentration ratio of more than 600 times raising the temperature of the receiver more than parabolic trough systems. Some systems use water as the heat transfer fluid. Molten nitrate based salts are being experimented with to be used as heat transfer medium as they have thermal energy-storage capability that might allow them to produce electricity at night [3]. Solar thermal systems as compared with solar cells and solar thermophotovoltaics are preferred for large-scale commercial plants because they not only offer electricity on demand, but also have the ability to store heat which can be later used generate electricity. This allows for greater flexibility and thus the choice for utility scale solar systems. This work is thus focused on solar thermal systems and developing coatings for making solar thermal receivers more efficient. 
For solar thermal systems, special coatings have to be applied on receivers for efficient energy conversion. The goal of the coatings is to have high solar light absorptance and low thermal emittance. This implies having low reflectance at wavelengths $(\lambda) \leq 2 \mu \mathrm{m}$, i.e. having high solar absorptance $(\alpha)$, and a high reflectance at $\lambda>2 \mu \mathrm{m}$. Infra-red (IR) reflectance wavelength requirement can change based on the temperature of operation of the receiver. Below in table 1 are the characteristics of the above two systems:

Table 1. Solar thermal systems

\begin{tabular}{|c|c|c|c|c|}
\hline & $\begin{array}{c}\text { Focusing } \\
\text { Element }\end{array}$ & $\begin{array}{c}\text { Concentration } \\
\text { Ratio }\end{array}$ & $\begin{array}{c}\text { Heat Transfer } \\
\text { Fluid }\end{array}$ & $\begin{array}{c}\text { Receiver } \\
\text { Temperature } \\
\text { Reached }\end{array}$ \\
\hline $\begin{array}{c}\text { Parabolic Trough } \\
\text { System }\end{array}$ & Parabolic & $30-100$ & Synthetic oil & $370-400^{\circ} \mathrm{C}$ \\
\hline $\begin{array}{c}\text { Central Tower } \\
\text { Shaped mirrors }\end{array}$ & Heliostats & $>600$ & Water & $450-550{ }^{\circ} \mathrm{C}$ \\
\hline
\end{tabular}

\subsection{Methods for achieving spectral selectivity}

The term spectral selectivity is used to describe the spectral behavior of a coating because it has to have low reflectance in the solar spectrum and high reflectance in the IR. Thus the material has a selective nature in reflectivity for different parts of the electromagnetic spectrum. In figure 5, we show solar spectrum and blackbody radiation at $450{ }^{\circ} \mathrm{C}$. As the sunlight is concentrated on the receiver, the temperature of the receiver rises and it radiates heat. The blackbody radiation is primarily in the IR spectrum and having high reflectance in the IR will 
prevent this heat from radiating. Therefore, the emittance $(\varepsilon)$ in the IR spectrum needs to be low and will result in higher conversion efficiency.

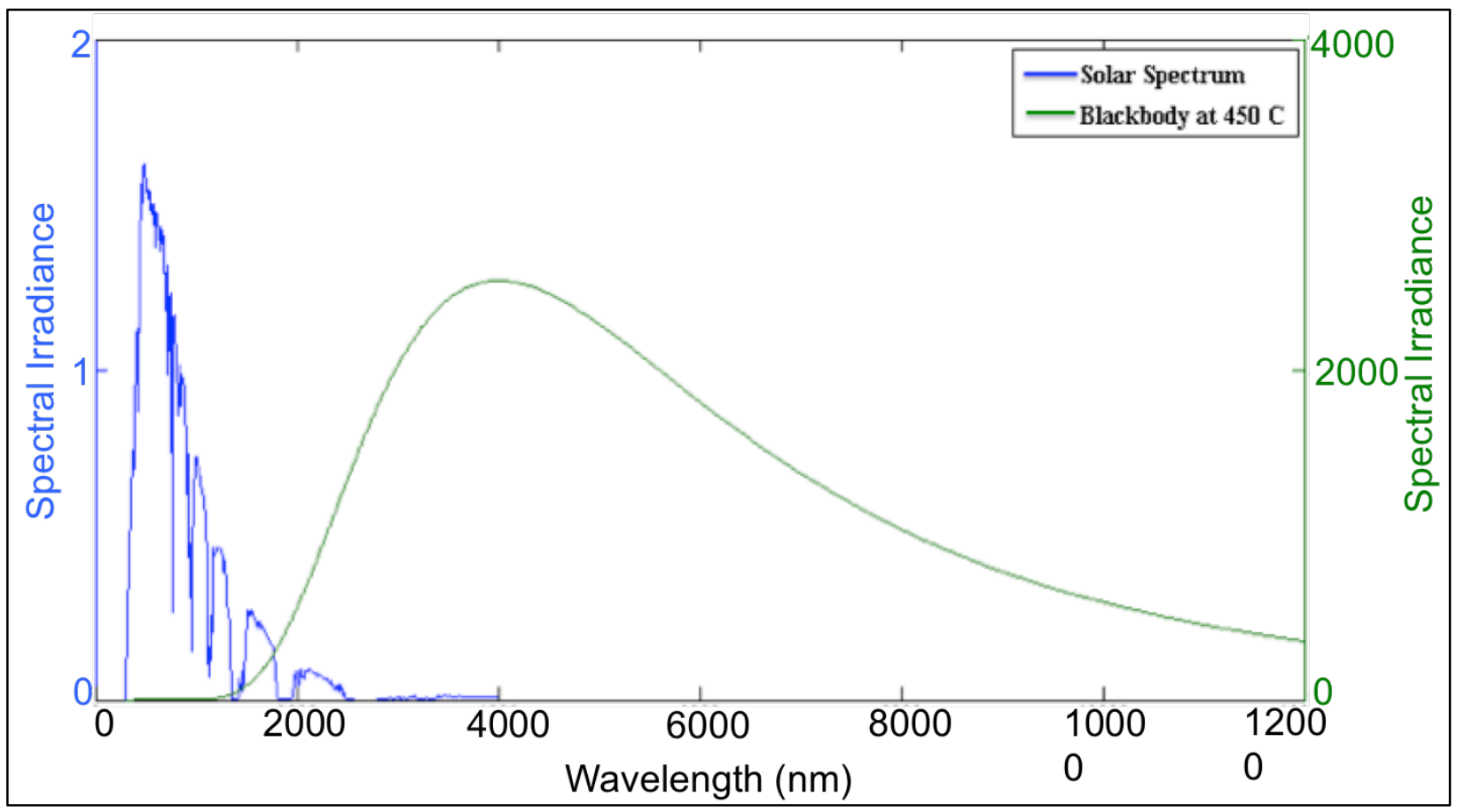

Fig 5. Solar spectrum and blackbody irradiance at $450{ }^{\circ} \mathrm{C}$

Several techniques have been used to develop spectral selective coatings and they are described below:

a. Intrinsic absorbers: Materials that have intrinsic optical property to achieve spectral selectivity at temperatures higher than $650{ }^{\circ} \mathrm{C}$, are not found in nature. As seen in figure 6 , using bulk material cannot give both, high absorptance in solar and high reflectance in IR spectrum. Metals like gold, silver, etc. have low $\varepsilon$ but also a very low $\alpha$. Silicon and germanium have been used as absorber surfaces [6] but exhibit poor performance in the IR 
spectrum. Antireflection (AR) coatings such as $\mathrm{SiO}_{2}, \mathrm{TiO}_{2}, \mathrm{Si}_{3} \mathrm{~N}_{4}$, and $\mathrm{Al}_{2} \mathrm{O}_{3}[6]$ have been used to increase $\alpha$, however keeping low $\varepsilon$ has remained a challenge. Other issues with using intrinsic absorbers are that they are expensive to manufacture and they degrade at high temperatures.

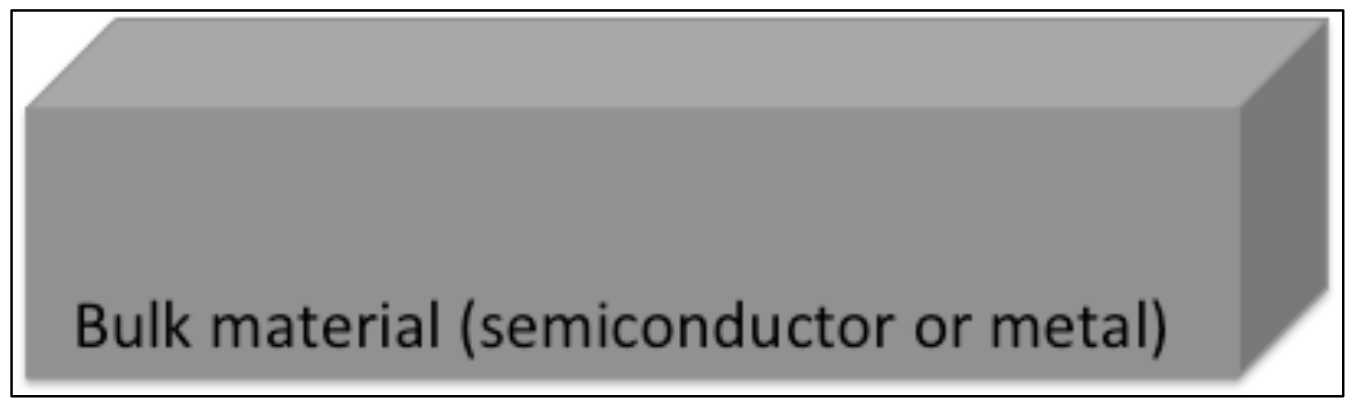

Fig 6. Bulk material as absorber surfaces.

b. Ceramic - metal composite (cermet): Cermet is a mixture of metallic particles in a dielectric host. The cermet layers act as absorbers in the solar region [6] and are deposited onto a base metal layer that acts as an IR reflector to keep the emittance low. In addition, anti-reflection layer is also added for reducing front surface reflection and to maintain thermal stability. Figure 7 shows a multilayer cermet coating. In order to increase solar absorptance, researchers have experimented with varying metal content, multiple layers of cermets, and metal particle sizes. Single layer cermets and multilayer cermets, behaving as graded index medium, have been used by researchers [7] to achieve high solar absorptance. $\mathrm{Cr}^{-} \mathrm{Cr}_{2} \mathrm{O}_{3}$ based cermet was fabricated using electroplating and achieved $\alpha \sim 96 \%$ and $\varepsilon \sim 25-35 \%$ when heated to $300{ }^{0} \mathrm{C}$ in vacuum [8]. W- $\mathrm{Al}_{2} \mathrm{O}_{3}$ cermet prepared by sputtering achieved $\alpha \sim$ $93.9 \%$ and $\varepsilon \sim 12 \%$ at $400{ }^{\circ} \mathrm{C}$ after being heated in vacuum at $580{ }^{\circ} \mathrm{C}[9] . \mathrm{Ni}-\mathrm{Al}_{2} \mathrm{O}_{3}$ cermet fabricated using sputtering technique achieved $\alpha \sim 94 \%$ and $\varepsilon \sim 7 \%$ at $100{ }^{\circ} \mathrm{C}$ when heated in 
air at $500{ }^{0} \mathrm{C}$ [7]. Challenges with cermet structures are related to high temperature degradation in air starting at $500{ }^{0} \mathrm{C}$ since cermets are susceptible to metal agglomeration, inter-diffusion, cracking, and delamination [7]. Also, the manufacturing of these layers requires a vacuum environment and precise control of film thickness, contributing to considerable manufacturing expense.

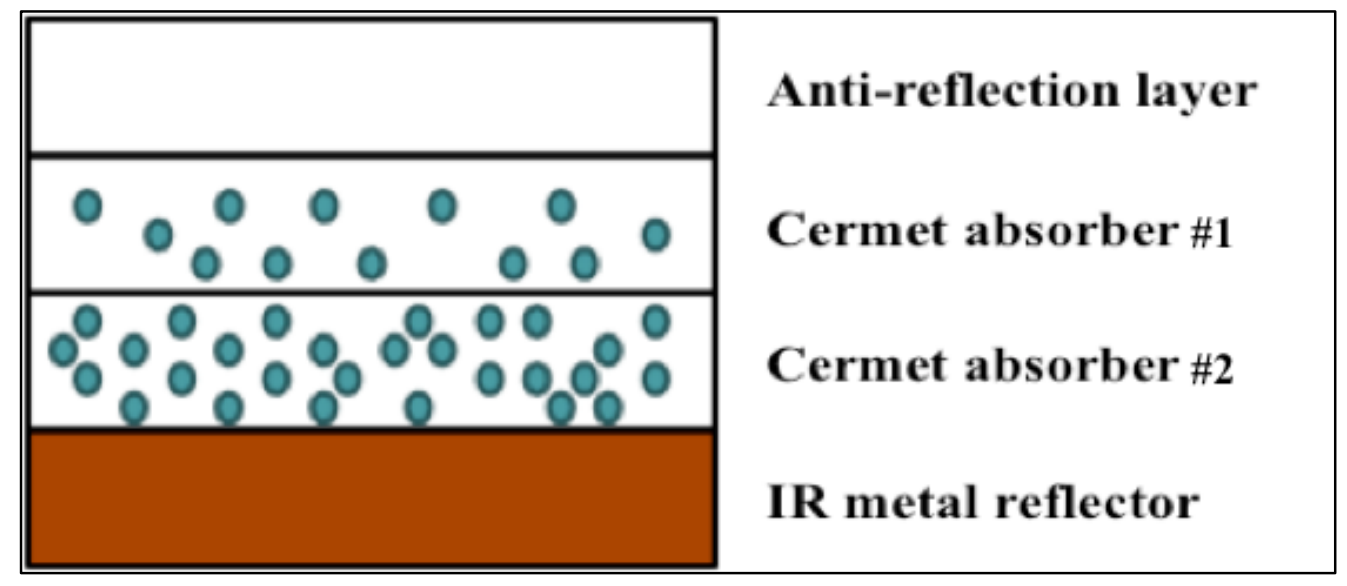

Fig 7. Cermet based spectral selective coating

c. Surface texturing: Surface texturing of materials as seen in figure 8 has been applied for solar energy applications for a long time. Surface texturing makes use of optical trapping of solar energy by multiple reflections of light, thus being partially absorbed at each reflection. This method makes it easier to achieve high solar absorptance but keeping emittance low and has been a challenge. Copper surface has been textured to achieve $\alpha \sim 90 \%$ and $\varepsilon \sim 10 \%$ at $300 \mathrm{~K}$ [10]. Laser texturing of copper was conducted to create periodical structures achieving $\alpha \sim$ $35 \%$ and $\varepsilon \sim 4 \%$ at $300 \mathrm{~K}$ [11]. Femtosecond laser processing of titanium and aluminum surface was conducted to achieve $\alpha>90 \%$ and emittance was not reported; however no heat treatment was done [12]. Submicron periodical cylindrical holes were fabricated on tungsten 
surface that achieved $\alpha \sim 82 \%$ and $\varepsilon \sim 15.9 \%$ [13] after heat treatment at $1200 \mathrm{~K}$ in vacuum. Chromium surface was textured to achieve $\alpha \sim 94 \%$ and $\varepsilon \sim 30 \%$ at $100{ }^{\circ} \mathrm{C}$ after being oxidized in air at $500{ }^{\circ} \mathrm{C}$ [7]. Nickel was sputtered that resulted in having surface roughness on the film [14] that had $\alpha \sim 80 \%$ and $\varepsilon \sim 10 \%$ at $200{ }^{\circ} \mathrm{C}$. The films were stable in air up to $300{ }^{\circ} \mathrm{C}$ but the performance went down after $500{ }^{\circ} \mathrm{C}$ due to increased oxidation of the film. Complex random structures after sputtering were formed on nickel and stainless steel with $\alpha$ $\sim 92 \%$ and $\varepsilon \sim 10-24 \%$ [14]. All these surfaces were fabricated using vacuum techniques adding to higher manufacturing costs. It can be seen that only some textured surfaces can be stable up to $\sim 500{ }^{\circ} \mathrm{C}$ in air while others can only sustain heat under vacuum. The issue with textured surfaces is that they tend to oxidize at high temperatures and also lead to modification in surface roughness, which causes a drop in performance.

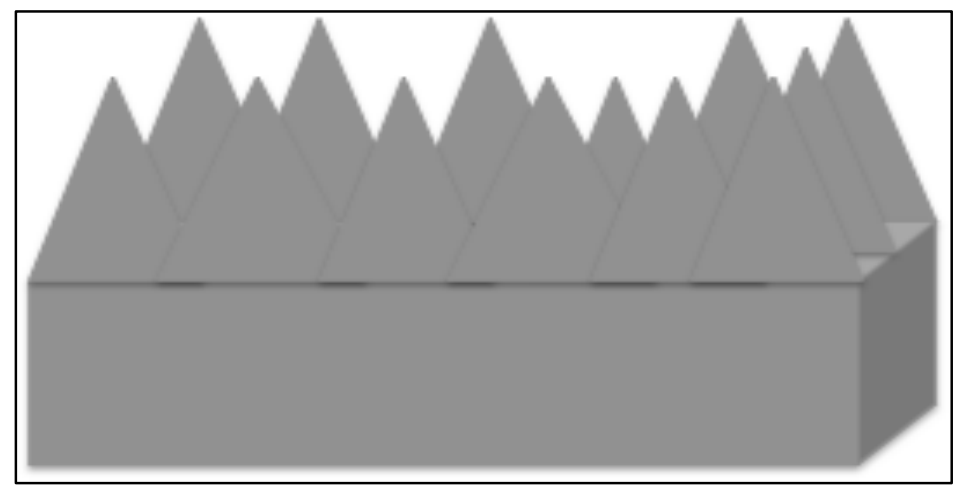

Fig 8. Surface texture for increasing solar absorptance (not to scale).

\subsection{Laser sintering of metal nanoparticles for spectral selectivity}

Sintering is a process in which particles of metals, ceramics, or polymers etc. are heated close to their melting point after which the particles join together and the density of the resulting film increases. There are two types of sintering: Solid state sintering (SSS) and liquid phase sintering 
(LPS). SSS occurs when the powder is densified wholly into a solid state at the sintering temperature, while liquid phase sintering occurs when a liquid phase is present during sintering [15]. Below is a brief description of the two types of sintering:

a. Solid state sintering: SSS involves joining of particles due to high temperatures and there is mass diffusion amongst them that results in grain growth. During SSS viscosities are relatively high resulting in improper densification. Thus, employing SSS can sometimes create pores in the final structure that is why some researchers prefer LPS.

b. Liquid phase sintering (LPS): LPS involves the formation of a liquid phase that coexists with the solid particulate phase. There are two techniques used to introduce liquid phase sintering. One is to mix powders having different chemistries [14] and melting points. The interaction of two different powders will form a liquid phase during sintering. The second technique is to sinter a pre-alloyed powder. Heating at temperatures between solidus and liquidus temperatures will introduce a liquid phase during sintering. Figure 9 describes microstructural evolution during LPS [14]: LPS and melting/solidification of material are the phenomena responsible for bonding of particles. This property is utilized for surfaces and structures in direct laser sintering of metal particles.

The energy transfer from a laser beam to a metal surface is determined by plasma frequency. Metals have high reflectance for electromagnetic waves having frequency below the plasma frequency and have high absorptance for frequencies above the plasma frequency. This takes place due to the free electrons reradiating the incoming electromagnetic wave. Reflectance of 
metallic surfaces increase with increasing wavelength and therefore, using Nd:YAG $(1.06 \mu \mathrm{m})$ laser is more preferable than $\mathrm{CO}_{2}(10.6 \mu \mathrm{m})$ lasers. Due to higher absorption with the metal, Nd:YAG laser leads to a better energy coupling with the material. Light absorption causes the intensity of the light to decay with depth at a rate determined by the material's absorption coefficient [16].

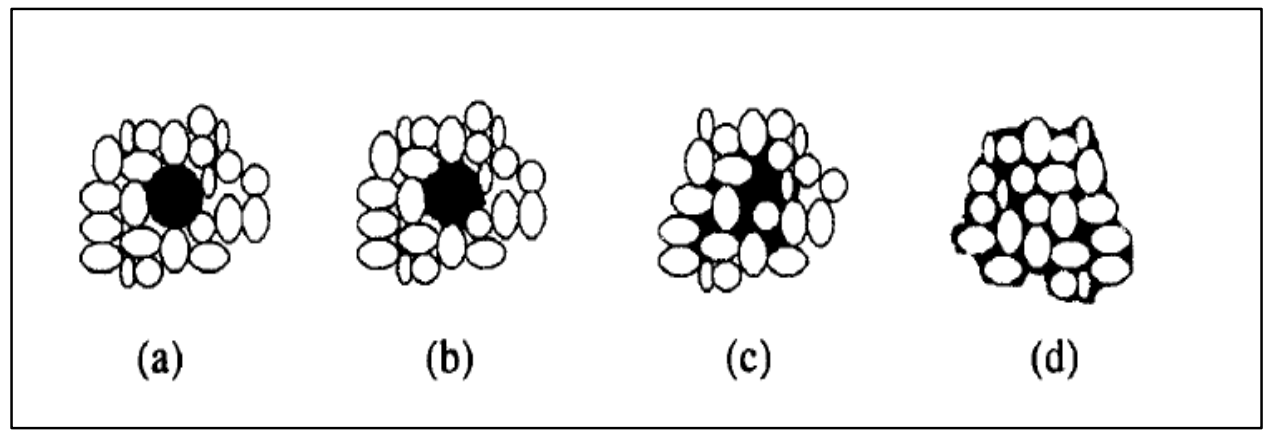

Fig 9. Microstructural evolution during LPS: (a) Low melting particle is surrounded by high melting particles, (b) low melting point particle starts melting, (c) the melt spreads, and (d) rearrangement of particles in a dense liquid-solid agglomerate

The intensity, I, decays exponentially within the material with depth $\mathrm{z}$ according to the BeerLambert law as follows:

$$
I(z)=I_{0} e^{-\gamma z}
$$

where $\mathrm{I}_{0}$ is the intensity right at the surface after reflection losses, $\gamma$ is material's absorption coefficient, and $\mathrm{z}$ is the depth with which the intensity I(z) decays exponentially. For example, in the case of most metals, the absorption depth is on the order of $10 \mathrm{~nm}$ when illuminated via UV 
radiation. Hence choosing wavelength with short absorption depths allows for local modification of the surface properties without affecting the bulk.

Laser sintering involves using a laser as a heat source to partially melt the particles and cause them to bind together in order to form a continuous sintered layer. Using a pulsed laser has advantages over using a continuous wave laser, since sintering can be achieved at lower average power levels. During sintering, high repetition rate of laser pulses causes multiple pulses to overlap onto a small spot resulting in partial melting and then consolidation. The temperature of the particles during pulsed laser interaction is what governs the melting of the surface of particles leading eventually to binding. The heat flow equation can be given as follows [17]:

$$
\rho C \frac{\partial T}{\partial t}-\nabla \cdot(k \cdot \nabla T)=f
$$

where $\rho$ is the density, $C$ the heat capacity, $k$ the thermal conductivity and $f$ the heat source [15]. In metals, the light is absorbed within a depth of the order of few nm [18]. This energy is absorbed by the free electrons and is then transferred to the lattice via collisions raising the temperature of the bulk. Since it's a powder and not a bulk metal, the penetration depth of light in the powder agglomerate can be on the order of microns. The resulting sintered surface and its morphology are dependent on the initial size of the particles and the thickness of the powder layer. The surface roughness formed on the sintered layer is used to achieve spectral selectivity and this roughness can be tailored to achieve high $\alpha$ and low $\varepsilon$.

Sub-wavelength structures on metal surfaces are highly desired as they increase solar absorptance as the surface behaves like graded index medium, providing antireflection properties [13]. For a desirable CSP receiver coating, the reflectance in the IR can be kept very high since 
IR wavelengths are much longer than the dimensions of surface roughness and therefore the surface appears smooth and radiates as a flat surface.

Therefore, the goal is to achieve sub-wavelength random surface roughness on sintered layer that can be spectrally selective and also be thermally stable. The laser heating of tungsten nano particles to form a sintered layer having surface roughness ranging from sub-microns to a couple of microns was investigated and it has achieved high spectral selectivity and thermal stability.

\subsection{Research objectives and plan}

The objective of this research is to investigate laser sintering of tungsten micro/nano particles to control the surface morphology so that spectral selective surfaces for concentrated solar power receivers can be achieved. Laser sintering technique was employed to form a continuous bonded layer and control of its surface roughness to achieve spectral selectivity. The effect of laser processing parameters on sintering were studied and optimized to attain a high performance coating. Surface characterization, optical and infrared measurements, and thermal stability of the coatings at high temperatures were investigated. To further increase solar absorptance, an antireflection film was deposited on the sintered layer and its performance was studied.

The goal was to demonstrate laser sintering of nanoparticles for solar thermal application along with fundamental understanding and basic measurements. The specific objectives of the research are listed below: 
- Literature review on CSP, spectral selective coatings, and laser sintering: Intrinsic absorbers, cermets, and surface texturing techniques that were used to achieve spectral selectivity. Laser sintering of metal particles was chosen to achieve spectral selectivity in this research

- Simulation of light absorptance and reflectance from rough and textured surfaces to understand the role of surface roughness on spectral selectivity and solar thermal performance

- Experimental setup for pulsed high power laser sintering of tungsten nanoparticles was designed and built

- Study effect of laser parameters on sintering of tungsten nanoparticles: The effect of laser power, pulse frequency, and scan speed on bonding and surface morphology

- Determine optimized laser processing conditions for sintering of tungsten nanoparticles to achieve $\alpha>90 \%$ and $\varepsilon \sim 16 \%$ at room temperature

- Investigate optical, infrared, morphological, and compositional properties of sintered tungsten coatings: Visible spectrum absorptance, FTIR reflectance, SEM, EDS, and XRD measurements are carried out

- Examine the effect of surrounding environment on laser sintering of tungsten nanoparticles: Sintering was carried out in presence of nitrogen atmosphere and under atmospheric pressure. Its effect on optical and IR properties before and after heat treatment were examined

- Investigated high temperature stability with optical measurement and various analytical techniques: Sintered samples were annealed at different temperatures up to $650{ }^{\circ} \mathrm{C}$ and 
the effect of temperature on optical and IR properties were studied. Thermal stability of the coatings were also examined using SEM, EDS, and XRD

- Study the effect of antireflection coatings on sintered tungsten films: Investigate the feasibility of sol-gel prepared $\mathrm{TiO}_{2}$ layer on laser sintered coatings. Measure its optical and IR properties

- Future work may involve long term stability and scaling up of this technique to be applied towards commercial solar thermal systems and areas where high temperature coatings are needed to operate without degradation, for example in nuclear industry. 


\section{Chapter 2. Modeling of light absorption and reflection}

\subsection{Electromagnetic wave interaction with surface roughness}

As mentioned earlier, surface texturing has been used to increase solar absorptance and this can be done by light trapping through multiple reflections by textures or when surface behaves as a graded index medium.

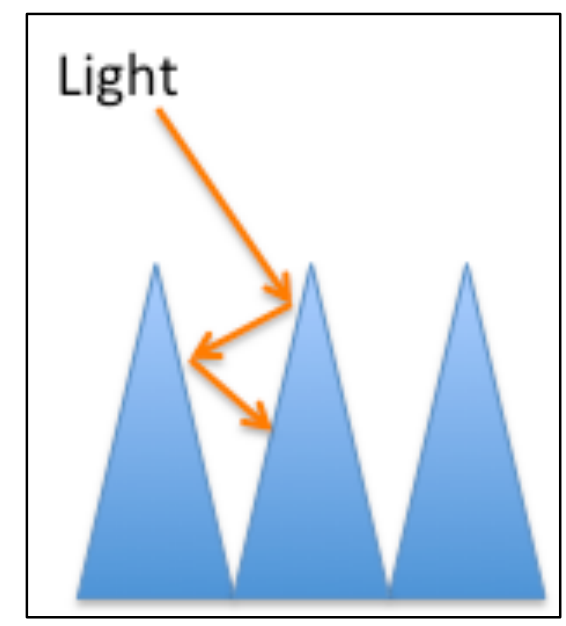

Fig 10. Light trapped by surface texture.

When spacing between surface texture features is larger than the wavelength, light absorption can be described by ray tracing. In light trapping, the goal is to increase the number of times light gets reflected by the surface texture as seen in figure 10 . When the surface roughness is less than the wavelength the surface behaves as a graded index medium thereby reducing front surface reflection. To achieve spectral selectivity, ideally surface roughness on tungsten needs to be sub-wavelength having dimensions $<500 \mathrm{~nm}$. This enables solar light to get absorbed but still 
have high IR reflectance. Since the wavelength of the IR radiation is almost $\sim 10$ times larger that the surface roughness, the surface starts to behave more like an effective medium [19] and thus high IR reflectance can still be achieved.

a. Effective Medium Theory (EMT): In EMT, a structure that is made from multiple materials is broken down into layers top-down and each layer is represented as a single material with an effective refractive index that is calculated using dielectric constant and filing factor of the constituent materials as shown in figure 11 . Since only a single effective refractive index is used, the represented material is homogenous and the scattering effects are not taken into account. Therefore, this method is only accurate for sub-wavelength sized textures that do not exhibit significant high-order diffraction [20]. In order to calculate effective medium, most common approaches are Maxwell Garnett, Lorentz-Lorentz, and Bruggeman theories. The Bruggeman theory is primarily used here due to the fact that it is accurate for a wide range of material ratios and is symmetric. Symmetric means that it does not matter which material is represented as an inclusion and which is specified as the matrix [20,21]. This is critical because in a dual-material system, either material can be considered as the inclusion.

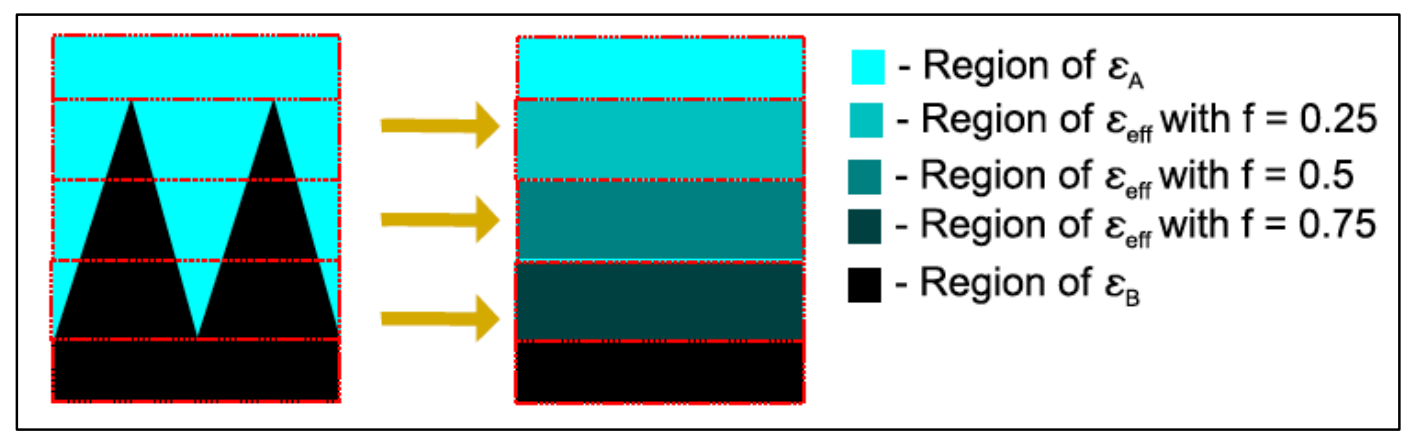

Fig 11. A sub-micron array of cone broken down into layers each having an effective index. 


$$
f \frac{\epsilon_{A}-\epsilon_{e f f}}{\epsilon_{A}+2 \epsilon_{e f f}}+(1-f) \frac{\epsilon_{B}-\epsilon_{e f f}}{\epsilon_{B}+2 \epsilon_{e f f}}=0
$$

The effective dielectric constant, originally derived by Bruggeman in his 1935 paper [22], is given in [23] as seen in equation 3 , where $\epsilon_{\text {eff }}$ is the effective dielectric constant, $\epsilon_{A}$ is the dielectric constant of material $\mathrm{A}, \epsilon_{B}$ is the dielectric constant of material $\mathrm{B}$, and $\mathrm{f}$ is the percentage of material having $\epsilon=\epsilon_{A}$. The effective refractive index of the material, $n_{\text {eff }}$, is equal to $\sqrt{\epsilon_{e f f}}$. These structures provide broadband absorption for textures with height greater than $0.4 \lambda$ and an inter-cone spacing less than $\lambda / 2 \mathrm{n}$, where $\mathrm{n}$ is the refractive index of the matrix material $[20,24]$. When using EMT to calculate spectral performance, $f$ is varied as a function of height in the structure, and the structure is broken down into many $(\sim 100)$ thin-film layers [20]. The reflectance can then be calculated using the transfer matrix method.

b. Finite difference time domain (FTDT) method: This work also uses the FDTD method to simulate the effect of various submicron textures on the reflectance spectrum of metal substrates. In an FDTD simulation, a solution is found by iteratively solving Maxwell's equations on a grid $[25,26]$. Central difference approximation is used to iteratively solve the time-dependent Maxwell's equations. A leapfrog method is then used to solve the discretized equations on a spatial grid called a Yee lattice. First the electric field vector components at each point in the lattice are solved, then the magnetic field vector components are solved for neighboring grid points for the next time-step, and so on [27]. FDTD simulations were run using an open-source program called the MIT Electromagnetic Equation Propagation 
(MEEP) package [28]. The FDTD method also takes into account scattering and resonant phenomena, allowing it to be accurate for feature sizes that are on the order of a wavelength. One disadvantage of the FDTD method (and numerical methods in general) is that errors can be introduced because of the discretization of phenomena.

c. Random texture vs. periodic structures: Creating structures on surfaces leads to increased absorptance but whether a periodic structure or a random one is preferable is addressed in this section. Many periodic arrangements exist that include photonic crystals, dielectric stacks, and 2D or 3D periodic textures [29,30]. Experimental results using grating structures on tungsten show absorption of over $90 \%$ in the solar spectral range [31]. Dielectric stacks can absorb $>90 \%$ of incident solar light while suppressing emissivity to be below $16 \%$ at $1750 \mathrm{~K}$ [32]. Submicron periodical cylindrical holes have been fabricated on tungsten surface and have achieved absorptance $\sim 82 \%$ and emittance $\sim 15.9 \%$ at $1200 \mathrm{~K}$ in vacuum [13]. Non-periodic textures or surface roughness were investigated for their use in achieving solar selectivity. The advantage of non-periodic structures is that it has a more broader absorption in the solar spectrum. The lack of periodicity in textures reduce the effect of Bragg peaks on the absorption spectrum [33] due to which relatively larger structures can retain their highly absorptive properties [2]. In addition to performance benefits, non-periodic surfaces are easier to fabricate as periodic structures require advanced manufacturing techniques. It becomes increasingly complex to generate structure with a periodicity smaller than the wavelength of absorbed light. In the case of a solar absorber, this requires structures on the order of $250 \mathrm{~nm}$ to avoid absorption peaks due to Bragg effects [33]. The non-periodic structures are able to achieve high absorptivity with structure sizes on the order of a micron. 
The absorptance of structures were simulated via finite difference time-domain (FDTD) method. Fig. 12 shows one of the random cone structures that was used for simulation. Fig. 13 shows simulated reflectance plot of these structures in the solar spectrum and near IR. It can be seen from Fig. 13a that increasing the height leads to increased absorptance due to a more gradual change in the refractive index from air to tungsten interface.

d. Effect of anti-reflection film: By having surface texture in addition to an antireflection film is ideal to having high solar absorptance. Figure 14 shows increased absorption by depositing $\sim 80 \mathrm{~nm}$ of $\mathrm{TiO}_{2}$ anti-reflection film. Anti-reflection coating not only helps to reduce front surface reflection, but it also prevents oxidation of the coatings at high temperatures in air thereby helping to maintain thermal stability and high performance.

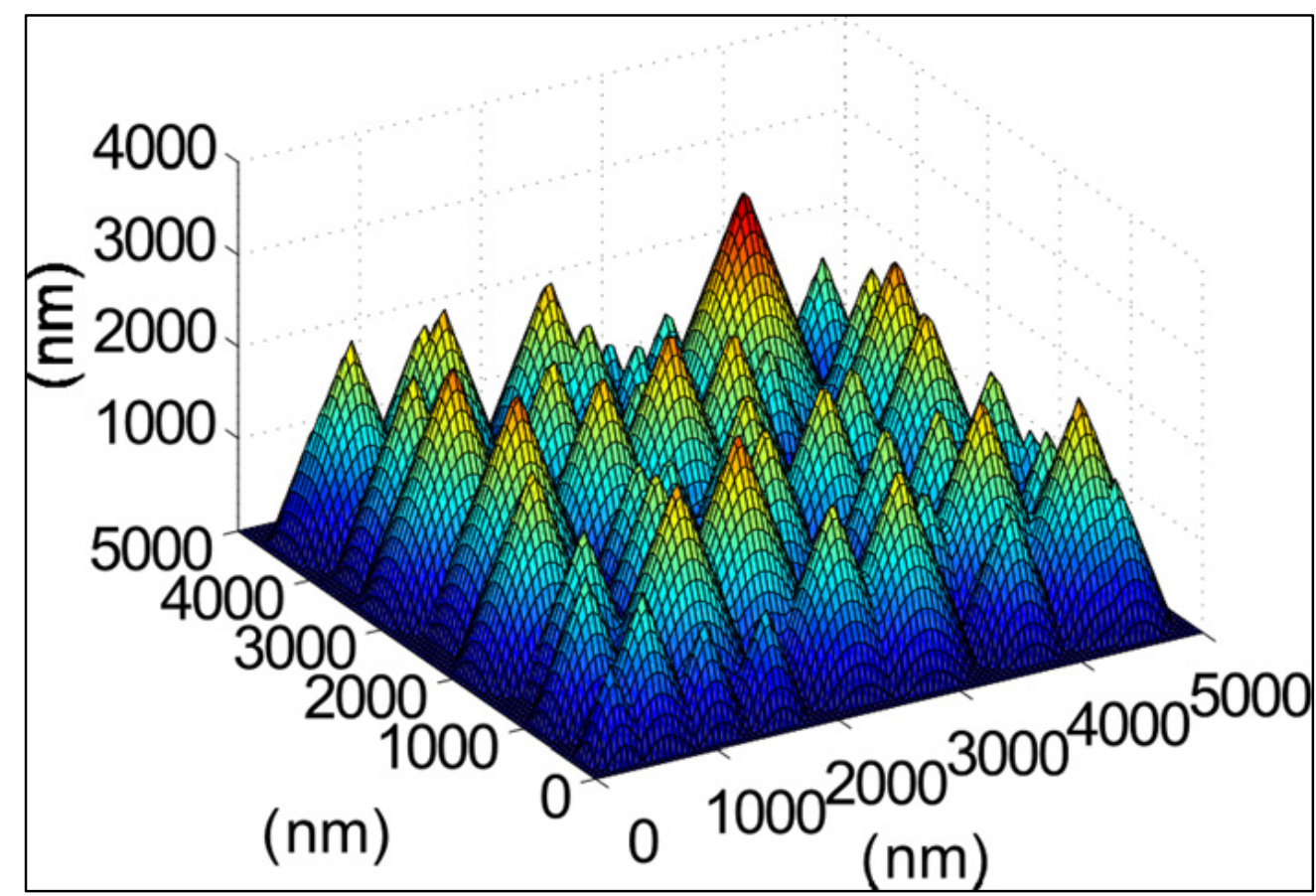

Fig 12. Cone structures on tungsten surface with random spacing and height [2]. 


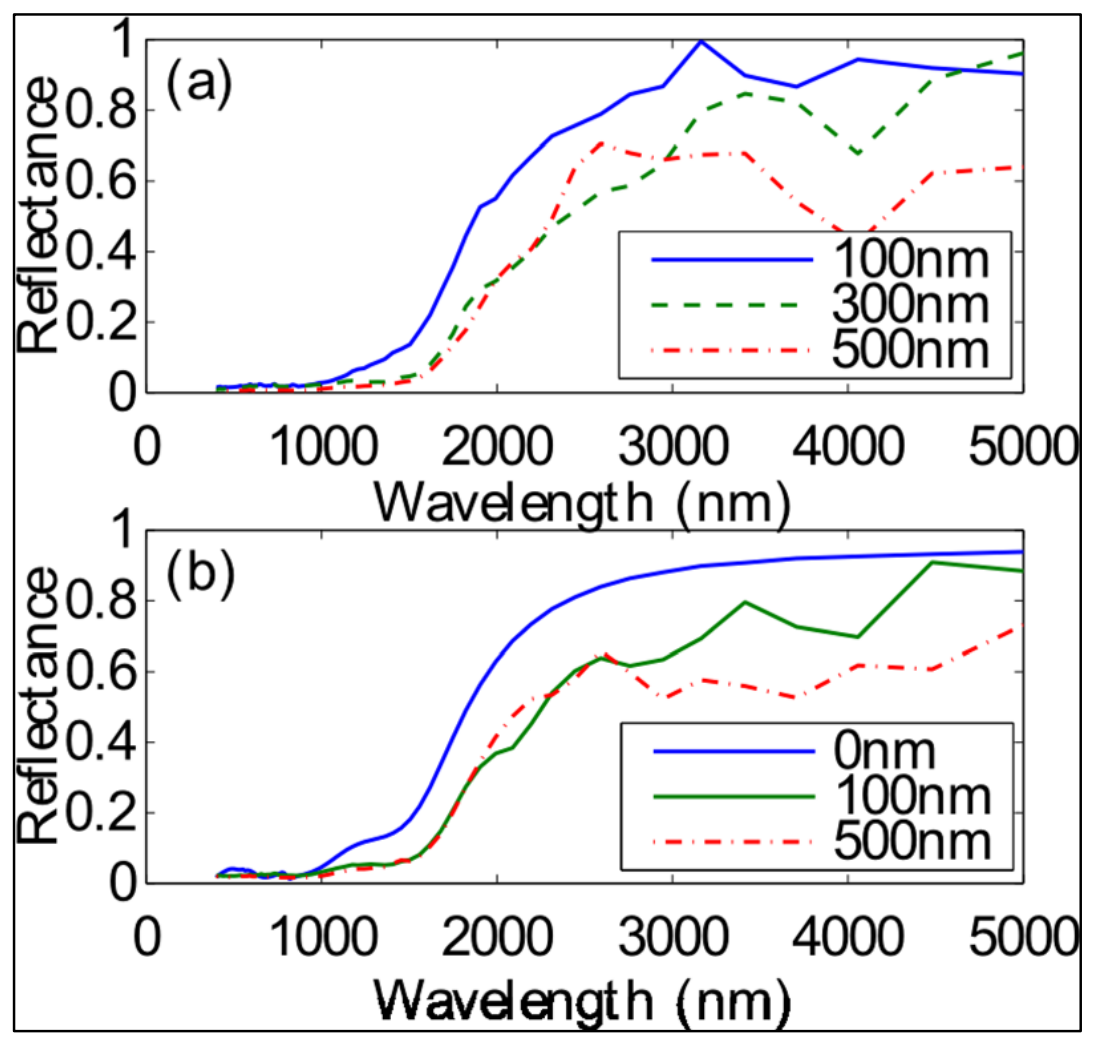

Fig 13. (a) Simulated reflectance for textured tungsten with different average heights.

(b) various distributions of cone periods [2].

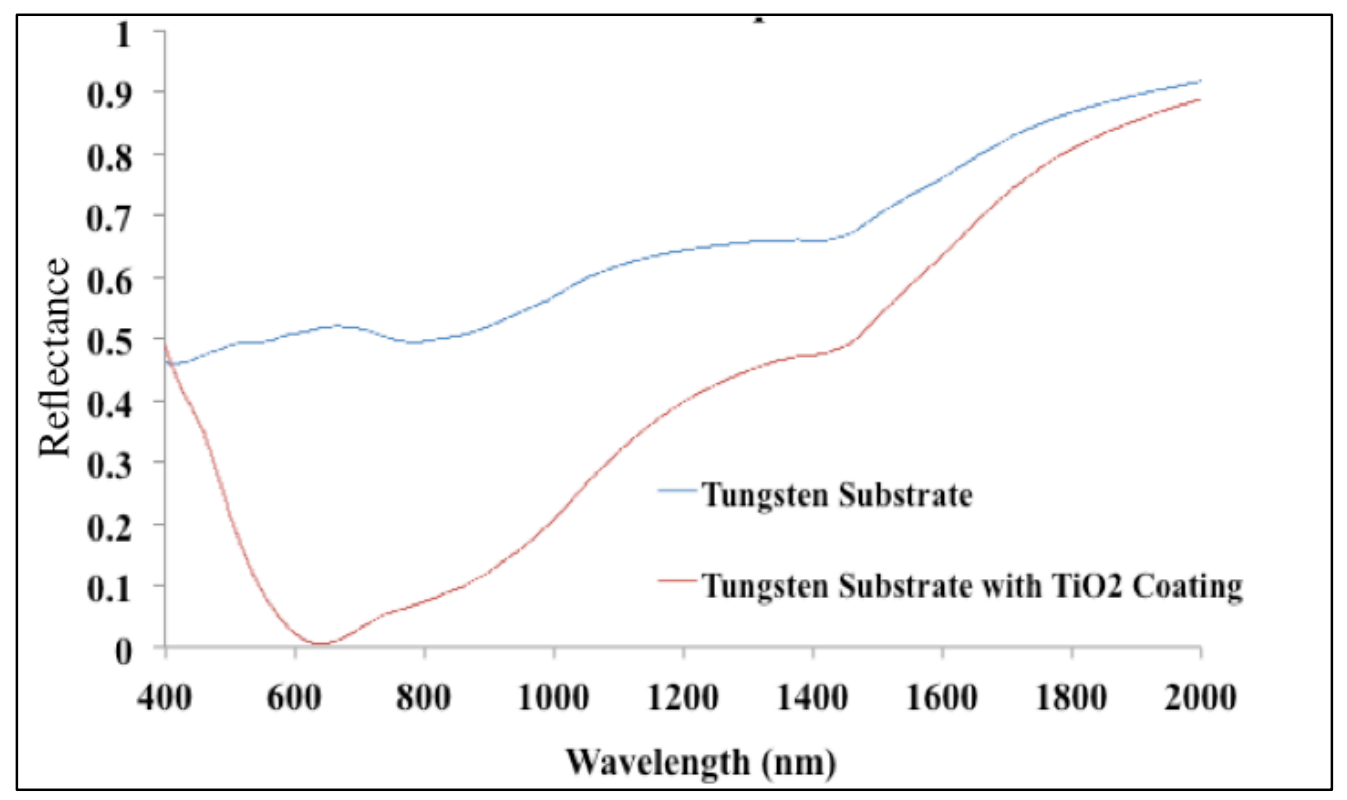

Fig 14. Reflectance for tungsten coated with $\mathrm{TiO}_{2} \mathrm{AR}$ coating. 
Thus the goal is to have surface roughness on sintered films that are sub-wavelength and which are coated with AR films to achieve high solar absorptance and also high reflectance in IR. Authors in [34] show that applying an antireflection layer on textured tungsten surface can increase solar absorptance $>90 \%$ and later in the report, we also present initial data of increased solar absorptance by applying an antireflection film on laser sintered coatings. 


\section{Chapter 3. Experiment: Laser Sintering of Tungsten}

\section{Nanoparticles}

\subsection{Experimental setup}

A pulsed fiber laser having wavelength of $1064 \mathrm{~nm}$ was used as a sintering source. Laser pulse width was $50 \mathrm{~ns}$, energy per pulse was 0.2 and $0.4 \mathrm{~mJ}$, and laser spot size was $\sim 120$ microns. A mirror and a lens were used to guide and focus the laser beam onto the sample on the scanning stage. Laser power, scan speed, and its frequency are the three parameters that were varied and numerous sintered films were fabricated.

Laser sintering experimental setup is shown in figure 15. Solar absorptance measurements for the fabricated sintered films were performed using an integrating sphere, supplied by Labsphere Inc. Reflectance measurements in the IR were done using a continuum IR microscope by Thermo Fisher Scientific Inc. The sample was illuminated by IR wavelengths ranging from 2.5 to 12.5 microns and the reflectance was measured from the sample surface. The IR microscope has the ability to collect reflected and scattered light over an angular range of $+/$ $35^{0}$ with respect to normal to the sample. X-ray diffraction measurements were conducted on sintered coatings using $\mathrm{CuK} \alpha$ radiation source to study the crystal structure. Scanning Electron Microscopy (SEM) and Energy Dispersive X - ray Spectroscopy (EDS) were conducted on sintered samples to study their morphology and elemental composition.

\subsection{Tungsten Nanoparticles}


Tungsten metal was chosen as the material of choice for sintering due to its optical and thermal properties. Since we need high solar light absorptance and high reflectance in the IR with

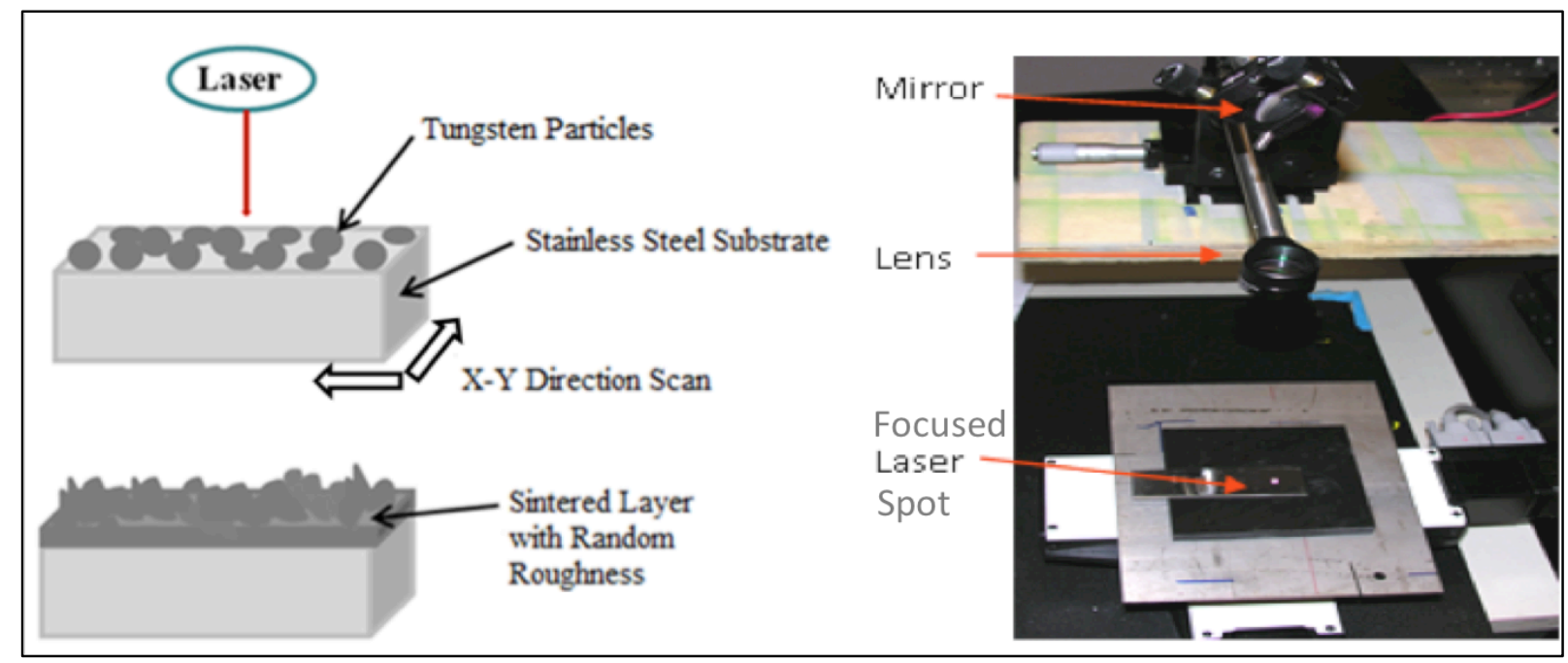

Fig 15. Experimental setup of laser sintering of tungsten nanoparticles.

materials having high melting point, metals turn out to be the preferred choice. However, not all metals have high solar absorptance. Tungsten as compared to all other metals has a fairly constant absorptance $\sim 48 \%$ in the solar spectrum and thus modifying the surface roughness can increase its absorptance even further. Bulk tungsten also has one of the highest reflectance in the IR spectrum, $\sim 97 \%$, and having the reflectance of laser sintered tungsten film approaching that of bulk tungsten is desired. In addition to optical properties, tungsten has the highest melting point, $\sim 3420{ }^{0} \mathrm{C}$, and using it for solar thermal where high temperatures are desired makes tungsten the most optimal choice. Hence choosing nanoparticles of tungsten was decided for laser sintering. Nanoparticles were ordered from SkySping Nanomaterials, Inc. and nano particles were chosen since the desired surface roughness needed to be $\sim 200-400 \mathrm{~nm}$ and 
having micro or bigger sized particles, the surface roughness that would form would be on a much bigger scale after sintering. Also, having too big particles can also result in relatively flatter surface morphology thus having lower solar absorptance. Most of the particles were between $80-100 \mathrm{~nm}$ but some were between $1-5$ microns. These particles are deposited onto a stainless steel (SS) substrate before sintering. SS is chosen as the substrate because it is the choice of receiver material for solar thermal applications due to its low cost and compatibility with HTF. The sample (tungsten particles on SS substrate) was then placed on an X-Y positioning stage for laser sintering.

\subsection{Nanoparticle deposition technique}

Various deposition techniques for nanoparticles were investigated and are presented below:

a. Dip coating: A beaker containing a solution of isopropyl alcohol (IPA) was mixed with tungsten nanoparticles. SS substrate was then dipped into the solution and withdrawn slowly. The sample was then kept aside to allow the IPA to evaporate, leaving only tungsten nanoparticles on SS. This approach proved ineffective because after IPA evaporation, tungsten particles were spread too far from each other, leaving large voids between them. Thus a continuous coating could not be formed because the particles were too far apart.

b. Drop casting in washer: A thin metallic washer was placed on SS substrate and the solution of IPA and tungsten nanoparticles was poured on SS in the center region of the washer using a dropper. After the IPA evaporated, the washer was removed and tungsten nanoparticles were close enough to be sintered. However, to form a sintered coating was difficult. The thickness of the coating was dependent on thickness of the washer therefore the layer of 
tungsten particles was too thick to be sintered effectively. This process caused a noncontinuous layer in some areas as bonding between tungsten particles and SS substrate did not occur.

c. Drop casting: The solution of IPA and tungsten nanoparticles was drop cast directly onto the SS substrate with a dropper. Two to three drops have to be placed in the same region on the substrate to ensure that tungsten particles were close enough for sintering after the IPA evaporated. This method has worked best compared to the other two methods and has been used for all the sintered samples. 


\section{Chapter 4. Characterization of Laser Sintered Tungsten}

\section{Films}

\subsection{Effect of laser process parameters}

Laser power, scan speed, and repetition rate of the laser were the parameters that were varied to examine the effect on sintering of tungsten nanoparticles. Repetition rate of the laser was varied from $30 \mathrm{kHz}$ to $80 \mathrm{kHz}$ to examine its effect on sintering tungsten nanoparticles. With higher frequency, more of a balling phenomenon was observed. Since the number of laser pulses per spot was too high an increasingly large amount of liquid can be produced that stays for a longer time [35]. The longer heat residence time leads to balling due to surface wetting phenomenon [36], therefore, $80 \mathrm{kHz}$ repetition rate was not selected. At lower frequencies, 30 $\mathrm{kHz}$ and $50 \mathrm{kHz}$, not much change in surface morphology was observed and as suggested in [35] a moderate repetition rate was recommended. Thus $50 \mathrm{kHz}$ was chosen as the repetition rate of choice. The other two parameters laser power and repetition rate were varied keeping the frequency fixed and their effect on sintering was examined.

a. Effect of laser power: The sample scan speed was fixed to $1 \mathrm{~mm} / \mathrm{s}$ and laser repetition rate was fixed at $50 \mathrm{kHz}$. In table 2, data are provided for conversion of laser power \% to laser power in watts.

Table 2. Laser power in watts 


\begin{tabular}{|c|c|c|c|c|}
\hline Laser Power (\%) & $\mathbf{2 0}$ & $\mathbf{4 0}$ & $\mathbf{6 0}$ & $\mathbf{8 0}$ \\
\hline Average Laser Power (watts) & 3.1 & 5.05 & 7.4 & 11.7 \\
\hline
\end{tabular}

The laser power was varied from $20 \%$ to $70 \%$ of the available $100 \%$ power from the laser. Below $20 \%$, laser power was not sufficient to melt the tungsten nanoparticles and bond them to SS, therefore a very discontinuous coating was formed showing areas of only partial bonding. As can be seen from Fig. 16a, the sintered region is discontinuous and SS substrate and melted tungsten both can be seen. The areas where tungsten nanoparticles did manage to get sintered were areas where the deposited tungsten layer was very thin. Thus even this low laser power was sufficient enough to melt and bind it to SS. Tungsten powder being deposited on SS is not of uniform thickness, so we have to choose the laser power such that we can still have a continuous sintered coating.

Laser power between $20 \%$ and $25 \%$ provided a thin continuous coating of sintered tungsten on SS. Figure 16b shows the SEM image for these sintered coatings with $20-25 \%$ laser power. This increase in laser power provided the increase in the total amount of deposited energy. This produced sufficient amount of liquid, improved the inter-track binding and was good enough to get desired sintered layer. Increasing the laser power to be between $30 \%$ and $40 \%$ caused a yellowish-white layer to form on the sintered region. This layer was of tungsten trioxide [37] as confirmed by X-ray diffraction measurements. The sintering was done in the presence of air and having laser power between $30 \%$ and $40 \%$ caused the formation of this opaque yellowish-white oxide layer of $\mathrm{WO}_{3}$ that was not suitable for solar thermal applications. Figure 16c shows SEM image of the oxide layer that was formed on the 
sintered layer under this power condition. The effect of further increase in laser power to $45 \%, 50 \%$, and $60 \%$ was examined. A continuous sintered coating with no opaque oxide layer was formed which meant that the temperatures were high enough to cause decomposition of the tungsten trioxide. A continuous layer with different surface morphology was observed due to different sintering kinetics and surface tension effects of the melt pool. The surface roughness was also on the larger scale $>5 \mu \mathrm{m}$. An increase in surface roughness has been observed with increase in average power [36] due to longer heat residence time having a tendency towards ablation thus resulting in larger structures. Fig 16d and 14e, show the SEM images for sintered coatings formed using laser powers between 45 $60 \%$ power. However, these power ranges could not be used since they resulted in greater surface roughness that caused low reflectance in IR and therefore higher emittance. Beyond $60 \%$, the laser power was too high and sintered layer exhibited cracks due to thermal stress as seen in figure 14f. Since the amount of deposited power was too high, ablation of the powder material takes place and the thermal stresses are enormous that can lead to cracks during the cooling phase [38].
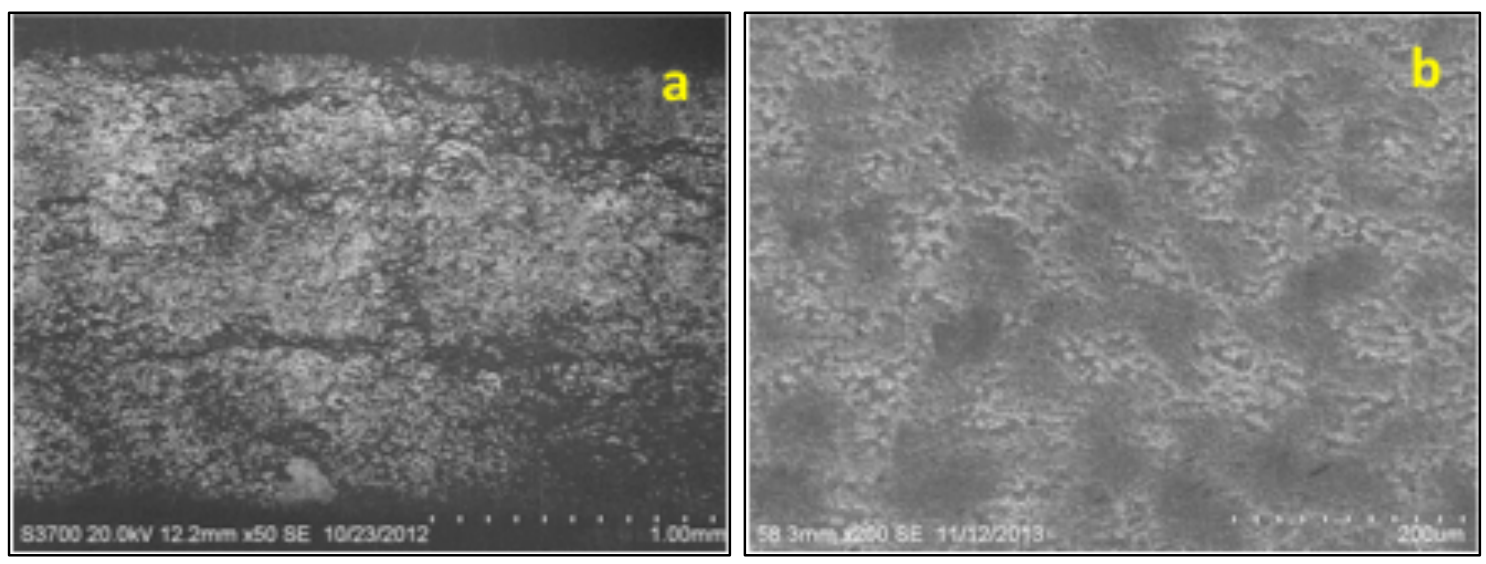

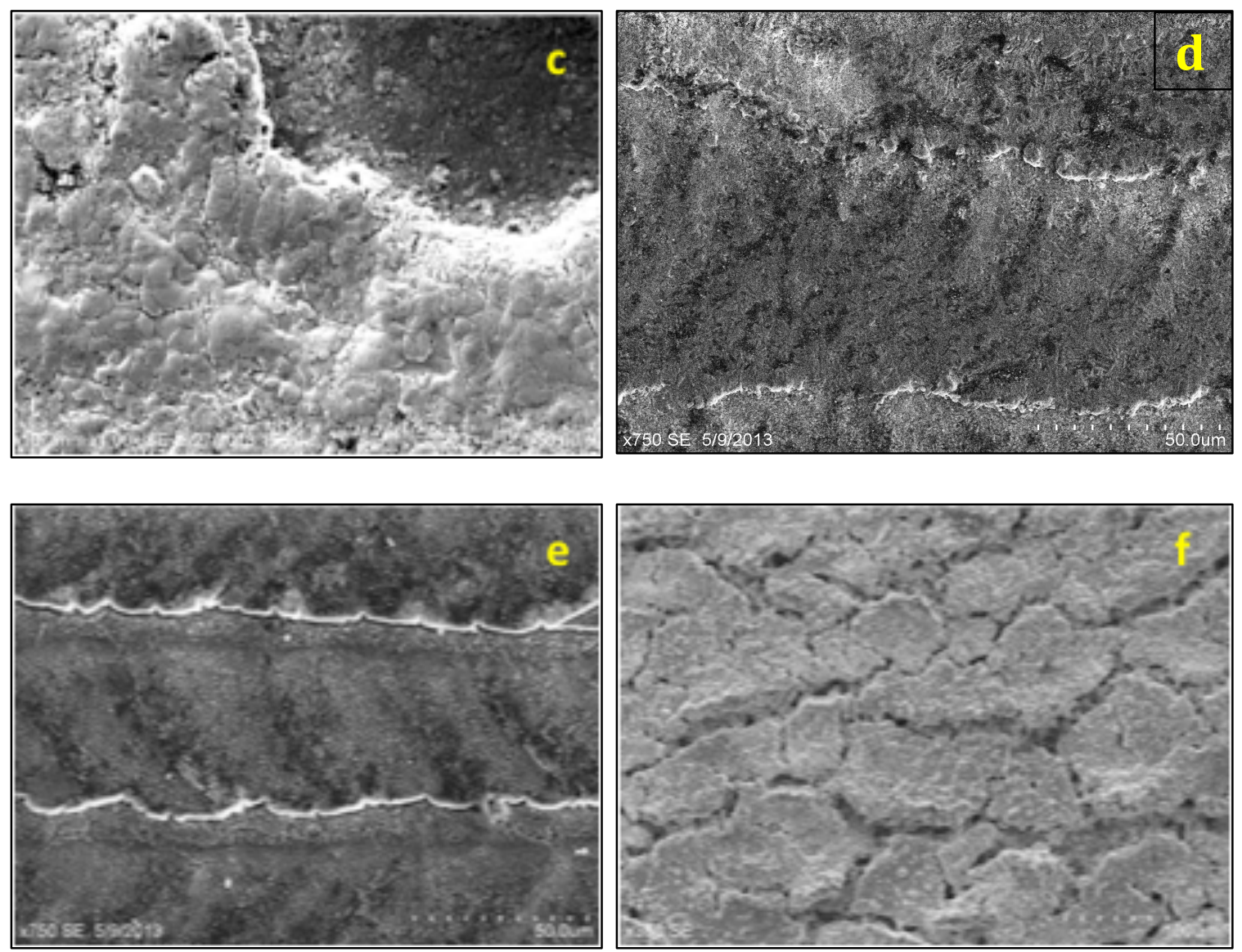

Fig 16. Laser sintered coatings with laser power (a) less than $20 \%$, (b) $20-25 \%$, (c) $30-$ $40 \%$, (d) $40-50 \%$, (e) $50-60 \%$, and (f) $>60 \%$.

b. Scan speed: The X-Y stage was scanned at various speeds ranging from $1 \mathrm{~mm} / \mathrm{s}$ to $10 \mathrm{~mm} / \mathrm{s}$ to determine the optimum speed to achieve a good bonded sintered layer. Below in table 3, solar absorptance for the sintered coatings that were formed with various laser powers and scan speed is presented. The numbers indicate the amount of solar absorptance obtained by performing only one scan under the laser. When the speed was $1 \mathrm{~mm} / \mathrm{s}$, coatings obtained using various laser powers were investigated and having laser power between $20-25 \%$ was chosen for giving desired morphological characteristics as described in section 3.3.a. When the speed was $3 \mathrm{~mm} / \mathrm{s}$, there was only partial bonding of the sintered layers up to $30 \%$ laser 
power. Yellowish-white oxide coating of tungsten trioxide was formed for laser powers between 30 and $40 \%$. Continuous sintered layers were formed for laser powers 50, 60, and $70 \%$. Further increase in laser power led to direct laser writing/texturing of SS which was not suitable. When the laser power gets too high, as mentioned earlier, considerable ablation of the powder can take place and the laser can directly start forming grooves in the substrate. When the speed was $5 \mathrm{~mm} / \mathrm{s}$, there was only partial bonding up to $40 \%$ laser power, tungsten trioxide formation for laser power between 40 and 50\%, and continuous sintered layers for 60 and $70 \%$ was observed. Finally, when the speed was increased to $10 \mathrm{~mm} / \mathrm{s}$, there was partial bonding of the layer up to $50 \%$ laser power, tungsten trioxide formation for $60 \%$, and continuous sintered layer at $70 \%$. As can be seen, the power levels at which $\mathrm{WO}_{3}$ starts to form has increased and that is due to the increase in scan speed. The deposited energy increases with laser power but decreases with scan speed, thereby producing similar condition for $\mathrm{WO}_{3}$ formation. The optimum speed was found to be $1 \mathrm{~mm} / \mathrm{s}$ since at other scan speeds the solar absorptance of the sintered coatings was not very high $(78-80 \%)$. In cases where similar solar absorptance to the ones produced using $1 \mathrm{~mm} / \mathrm{s}$ are achieved (for example having $3 \mathrm{~mm} / \mathrm{s}$ at 50 and $60 \%$ power, and $10 \mathrm{~mm} / \mathrm{s}$ at $70 \%$ power), IR reflectance was used for comparison. IR reflectance for sintered layers produced at these high scan speeds was much lower due to having larger surface roughness than the ones obtained using $1 \mathrm{~mm} / \mathrm{s}$ scan speed. Thus, even though solar absorptance is similar having low IR reflectance will result in a lower performance therefore, $1 \mathrm{~mm} / \mathrm{s}$ with laser power between 20 and $25 \%$ was chosen as the optimum laser sintering parameters.

\subsection{Morphological study by scanning electron microscopy (SEM)}


After varying all the laser process parameters, optimum conditions for the coating with best performance were finalized. Scan speed of $1 \mathrm{~mm} / \mathrm{s}$ and laser power of $20 \%$ was used to sinter the nanoparticle coating as that was the optimum as discussed in section 4.1. SEM image for the sintered coating after the first laser scan is show in figure 17. As concluded from this SEM image, the first laser scan was performed to form a continuous sintered coating without any voids. Solar absorptance of this coating was $\sim 70 \%$.

Table 3. Solar absorptance for different laser powers vs. scan speeds obtained by performing only one scan under the laser (' - ' means partial or no sintering)

\begin{tabular}{|c|c|c|c|c|}
\hline & \multicolumn{4}{|c|}{ Scan Speed } \\
\hline $\begin{array}{c}\text { Laser Power } \\
(\%)\end{array}$ & $1 \mathrm{~mm} / \mathrm{s}$ & $\mathbf{3} \mathrm{mm} / \mathrm{s}$ & $\mathbf{5 ~ m m / s}$ & $10 \mathrm{~mm} / \mathrm{s}$ \\
\hline$<20$ & - & - & - & - \\
\hline $20-25$ & $65-70 \%$ & - & - & - \\
\hline $30-40$ & Oxide Coating & Oxide Coating & - & - \\
\hline 50 & $\sim 80 \%$ & $\sim 78 \%$ & Oxide Coating & - \\
\hline 60 & $\sim 80 \%$ & $\sim 78 \%$ & $\sim 79 \%$ & Oxide Coating \\
\hline 70 & Cracks & $\sim 79 \%$ & $\sim 79 \%$ & $\sim 79 \%$ \\
\hline
\end{tabular}




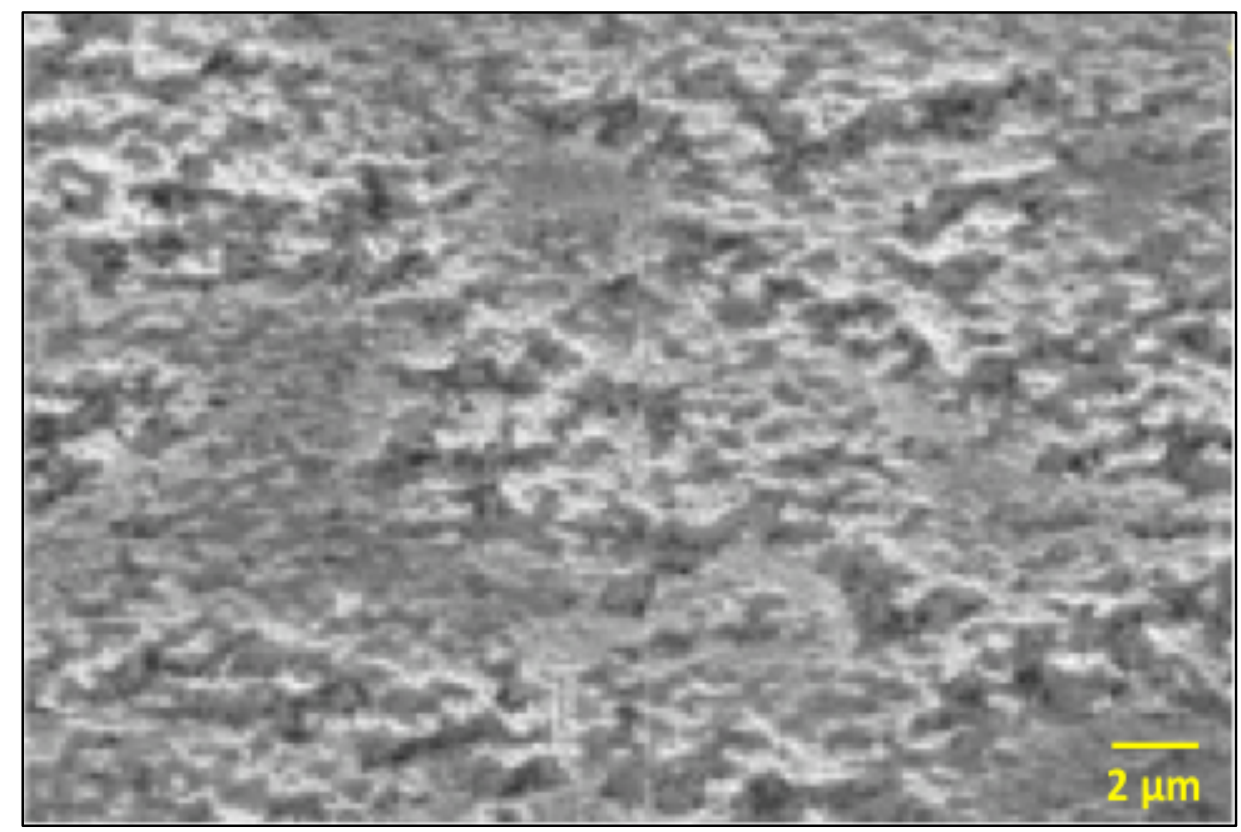

Fig 17. SEM image of laser sintered coating at $20 \%$ laser power.

Once this layer was sintered, a second scan on this sintered layer was done without any powder drop casting. Due to variation in coupling of laser energy to powder vs. sintered layer, different surface morphologies were observed. The goal was to create surface roughness on the sintered layer that would give high solar absorptance and thus the second scan was necessary to modify the surface roughness of the already sintered tungsten layer. Fig. 18 shows the SEM image of sintered layer using 30\% laser power and fig. 19 with using $35 \%$ laser power for second scan. As can be seen from the SEM images, the roughness formed on the sintered layers after the first and the second scan is quite different. The second laser scan with laser power $30 \%$ in fig. 18 modified the surface at random areas. The laser power was not high enough and due weak coupling with the already sintered layer having random roughness with different heights leads to modification only in some areas. However, the surface did get more rougher leading to having solar absorptance $\sim 75.6 \%$. Increasing the laser power to $35 \%$, also increased solar absorptance 
to $\sim 82 \%$. This can be explained by the SEM image in fig. 19 . The surface roughness starts to take some form of structure/pattern and the solar light now gets absorbed more.

Finally, increasing laser power to $40 \%$ produced a surface roughness with distinct peaks and valleys as seen in fig. $20 \mathrm{a}$ and b. This laser power was enough to modify the entire sintered coating uniformly and was chosen as the laser power for second scan. On this coating, surface roughness has some semi-periodicity and as seen in fig 20c, there is additional sub-micron roughness on those peaks. The combined effect of sub-micron roughness on semi-periodic structures, led to achieve highest solar absorptance of $\sim 90.3 \%$. This was our optimized coating and was used throughout the rest of the work for further investigation.

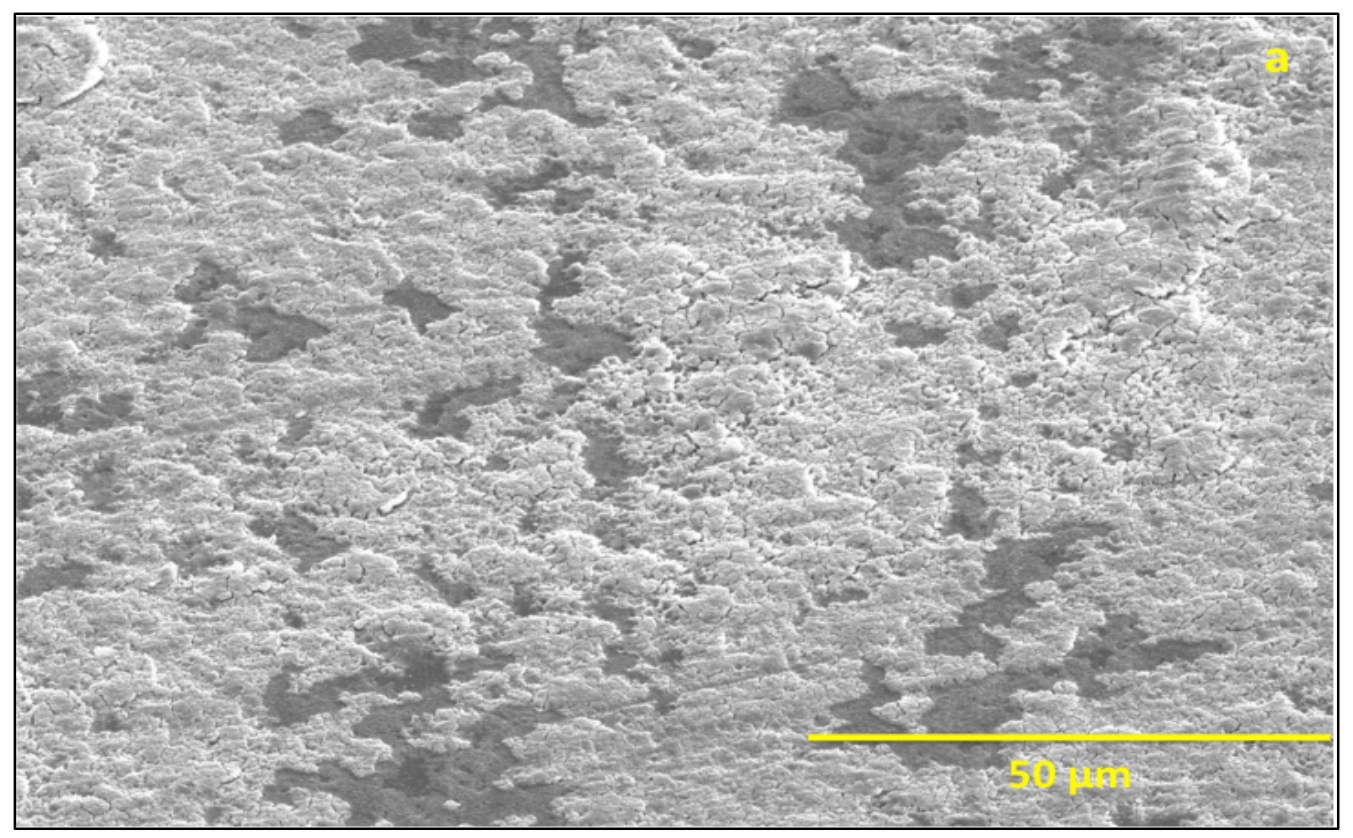




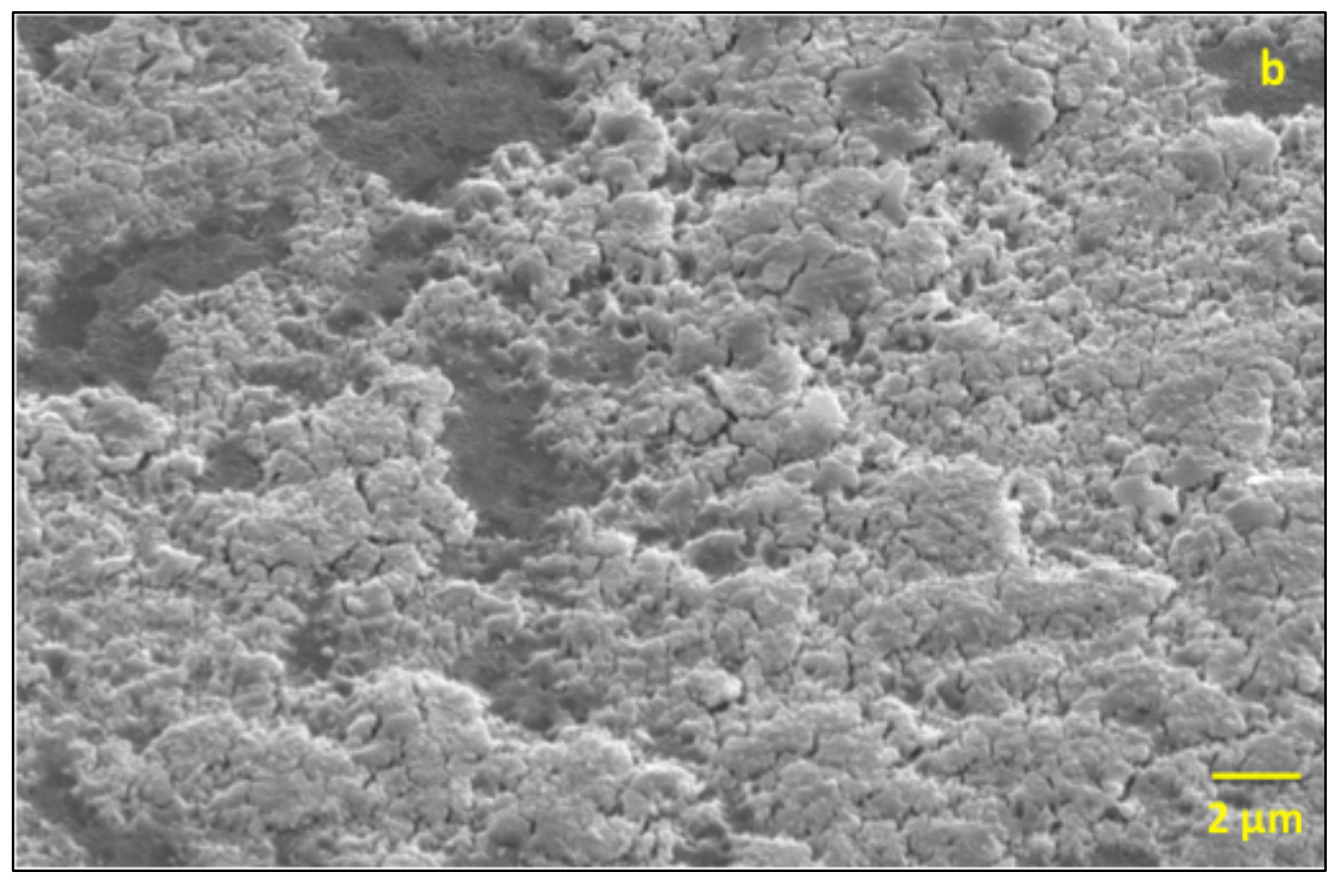

Fig 18. SEM images of sintered coating with $2^{\text {nd }}$ scan having laser power $30 \%$ (a) at lower magnification and (b) higher magnification

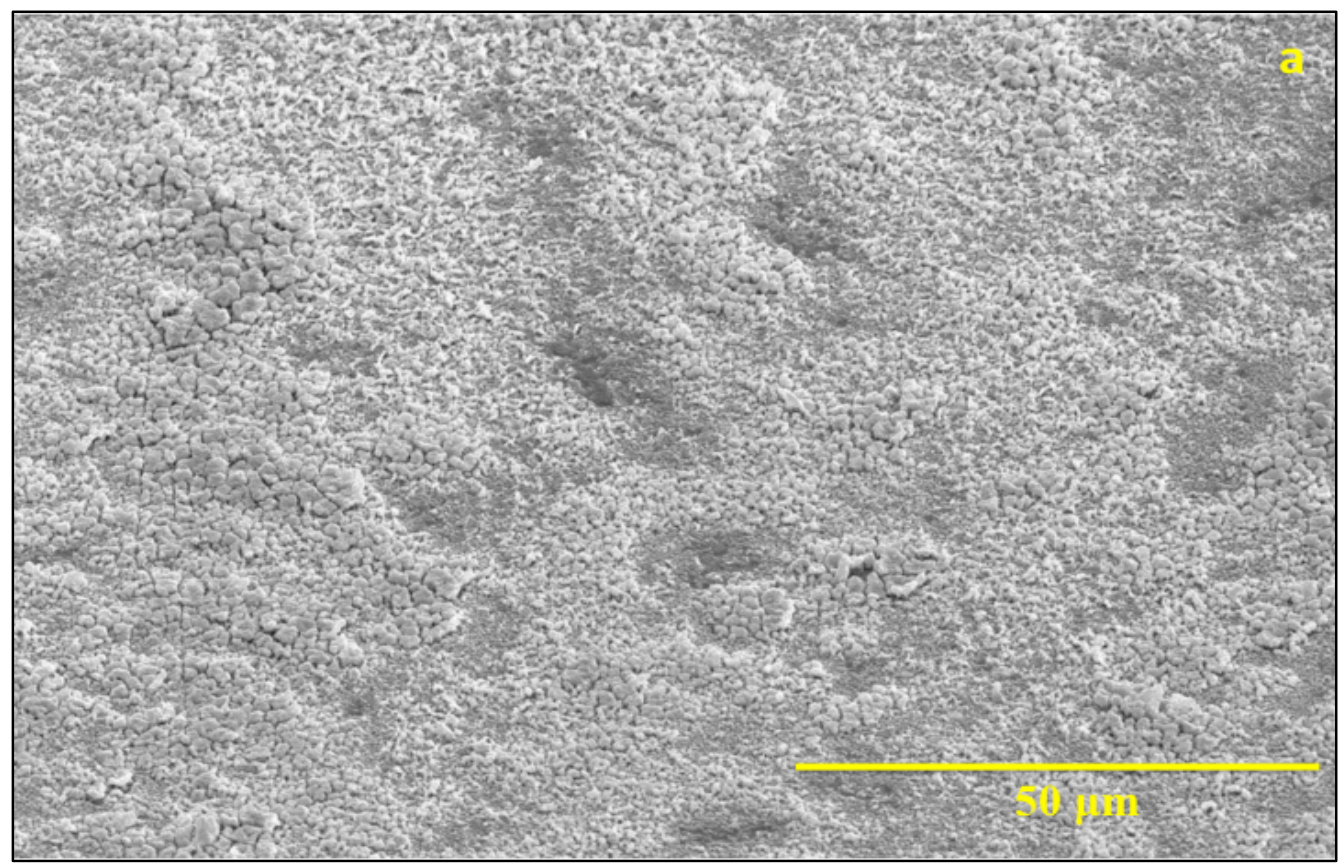




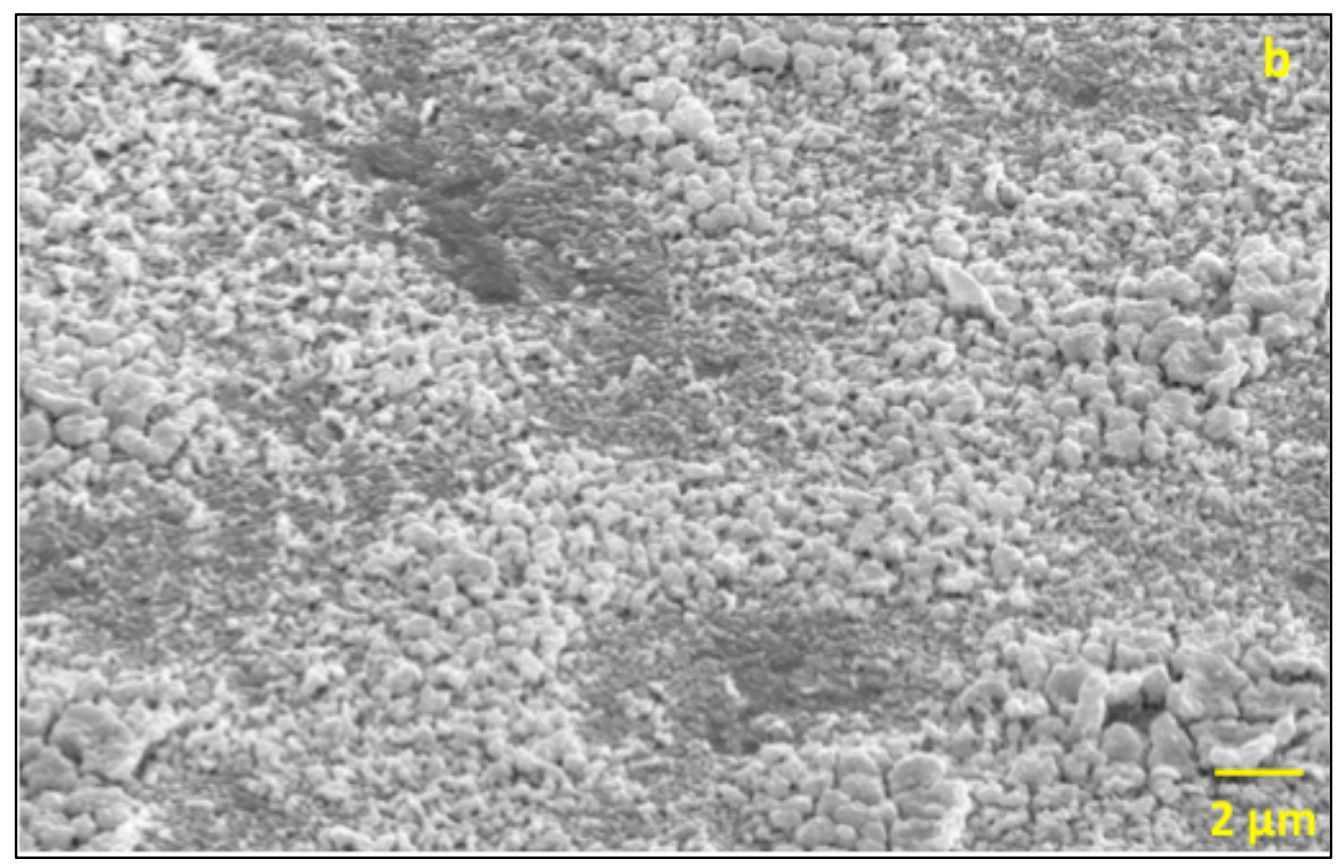

Fig 19. SEM images of sintered coating with $2^{\text {nd }}$ scan having laser power $35 \%$ (a) at lower magnification and (b) higher magnification

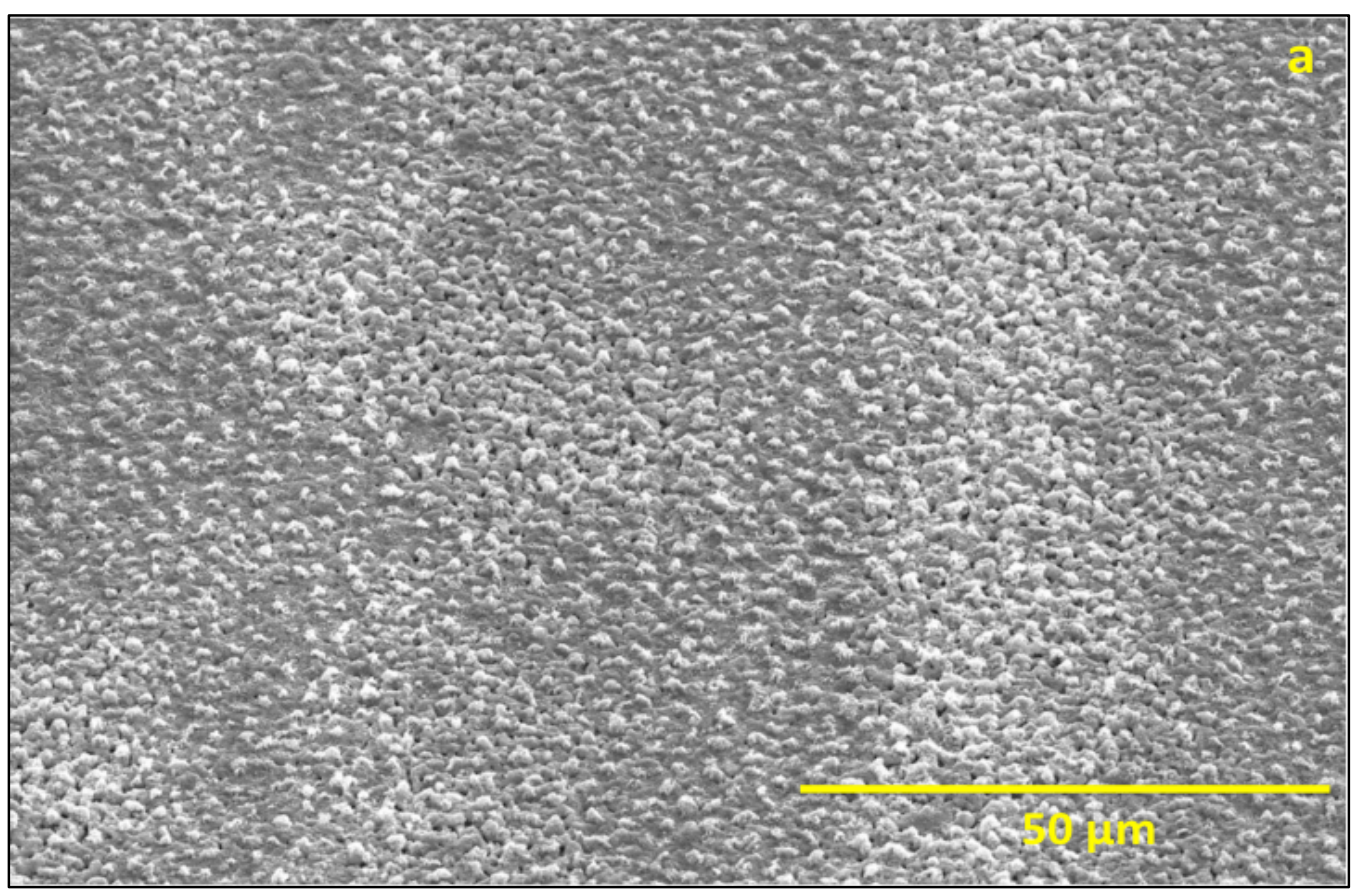



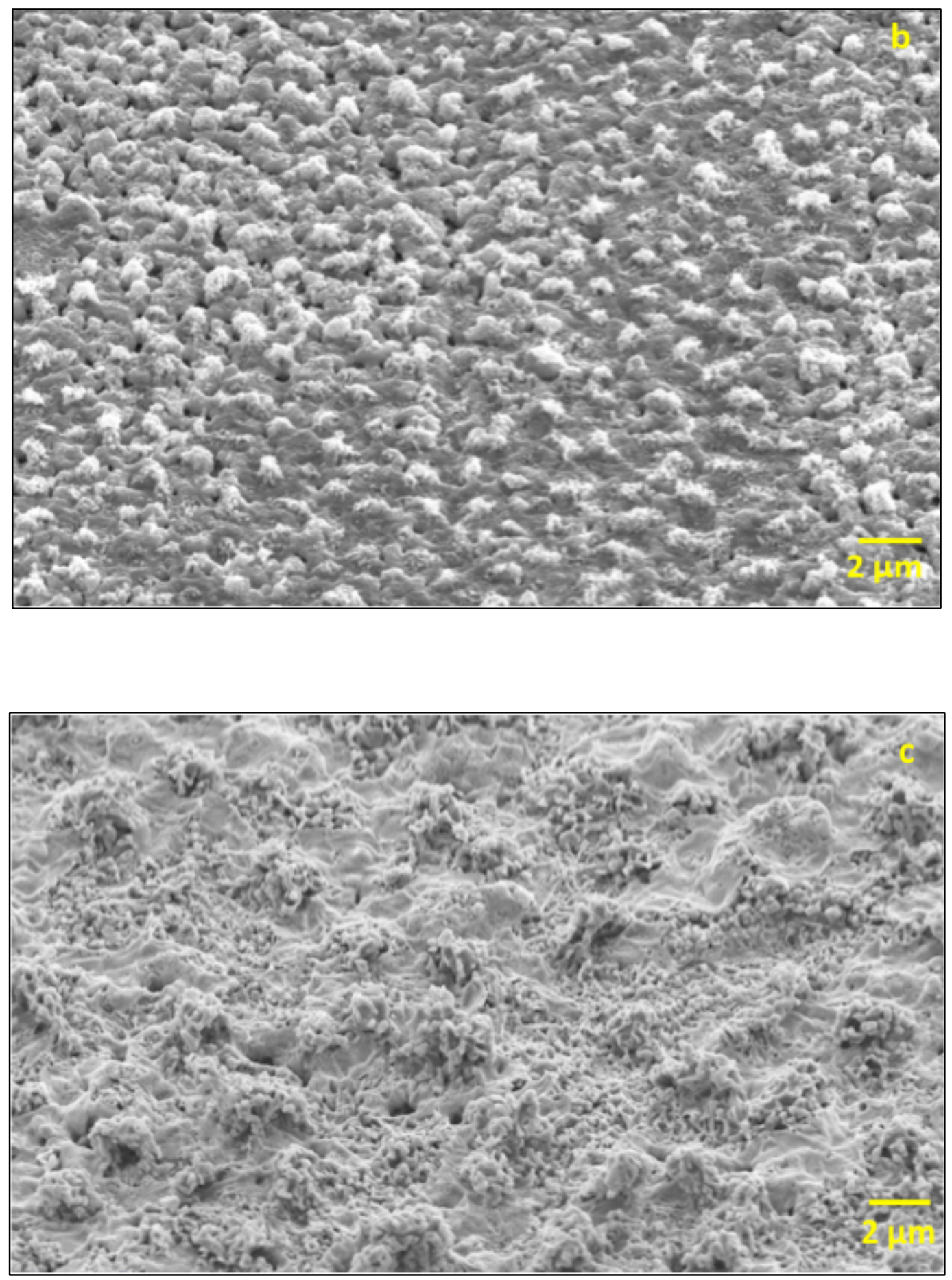

Fig 20. SEM images of sintered coating with $2^{\text {nd }}$ scan having laser power $40 \%$ (a) at lower magnification, (b) at higher magnification, \& (c) at highest magnification. 


\subsection{Composition study using energy dispersive x-ray spectroscopy (EDS)}

Elemental composition was found using EDS and for stainless steel substrate the weight (wt) percent composition was as follows: $18.2 \%$ chromium, $8.1 \%$ nickel, $1.44 \%$ manganese, $3.6 \%$ carbon, $0.3 \%$ silicon, and the remaining is iron. Elemental composition of the optimized laser sintered coating is shown in Table 4. It shows the EDS data for the sintered layer after the first laser scan using $20 \%$ laser power and after second laser scan using $40 \%$ power, respectively. After first laser scan with $20 \%$ laser power, the wt $\%$ of tungsten was 44.36 , iron was 31.20 , chromium was 9.38, nickel was 3.29 , and oxygen was 11.77. After second scan with higher laser power, the wt $\%$ of tungsten was 28.42 , iron is 38.66 , chromium is 9.21 , nickel is 3.19 , and oxygen is 20.52 . The decrease in the $\mathrm{wt} \%$ of tungsten is due to it being oxidized at high laser power since higher temperatures are reached. The variation in composition from location to location can be $\sim 5 \%$. Iron, nickel, and chrome are the elements present in SS. It can be seen that there was increased oxidation after the second scan as it is expected since higher temperatures are reached during the second scan with higher laser power.

Table 4. EDS data for sintered coating after $1^{\text {st }}$ and $2^{\text {nd }}$ laser scan

\begin{tabular}{|c|c|c|}
\hline & $\begin{array}{l}1^{\text {st }} \text { Laser Scan - } \\
20 \% \text { Power }\end{array}$ & $\begin{array}{l}2^{\text {nd }} \text { Laser Scan - } \\
40 \% \text { Power }\end{array}$ \\
\hline Tungsten & 44.36 & 28.42 \\
\hline Iron & 31.20 & 38.66 \\
\hline Chromium & 9.38 & 9.21 \\
\hline
\end{tabular}




\begin{tabular}{|l|l|l|}
\hline Nickel & 3.29 & 3.19 \\
\hline Oxygen & 11.77 & 20.52 \\
\hline
\end{tabular}

In order to further understand the composition of the sintered surface, we took a crosssection of the sample and looked at its elemental composition. Below in fig. 21 is the SEM image of the cross section. From fig. 21, we see a continuous bonded sintered layer on top of stainless steel substrate. Laser sintering has caused a very good bonding to the substrate without any discontinuity and has been used for further investigation. Fig. 22 shows profile of the elements present from the top down. Fig. 22a shows that tungsten (green color) is present only in the sintered layer and below is the SS substrate. The other elements chromium, iron, and nickel

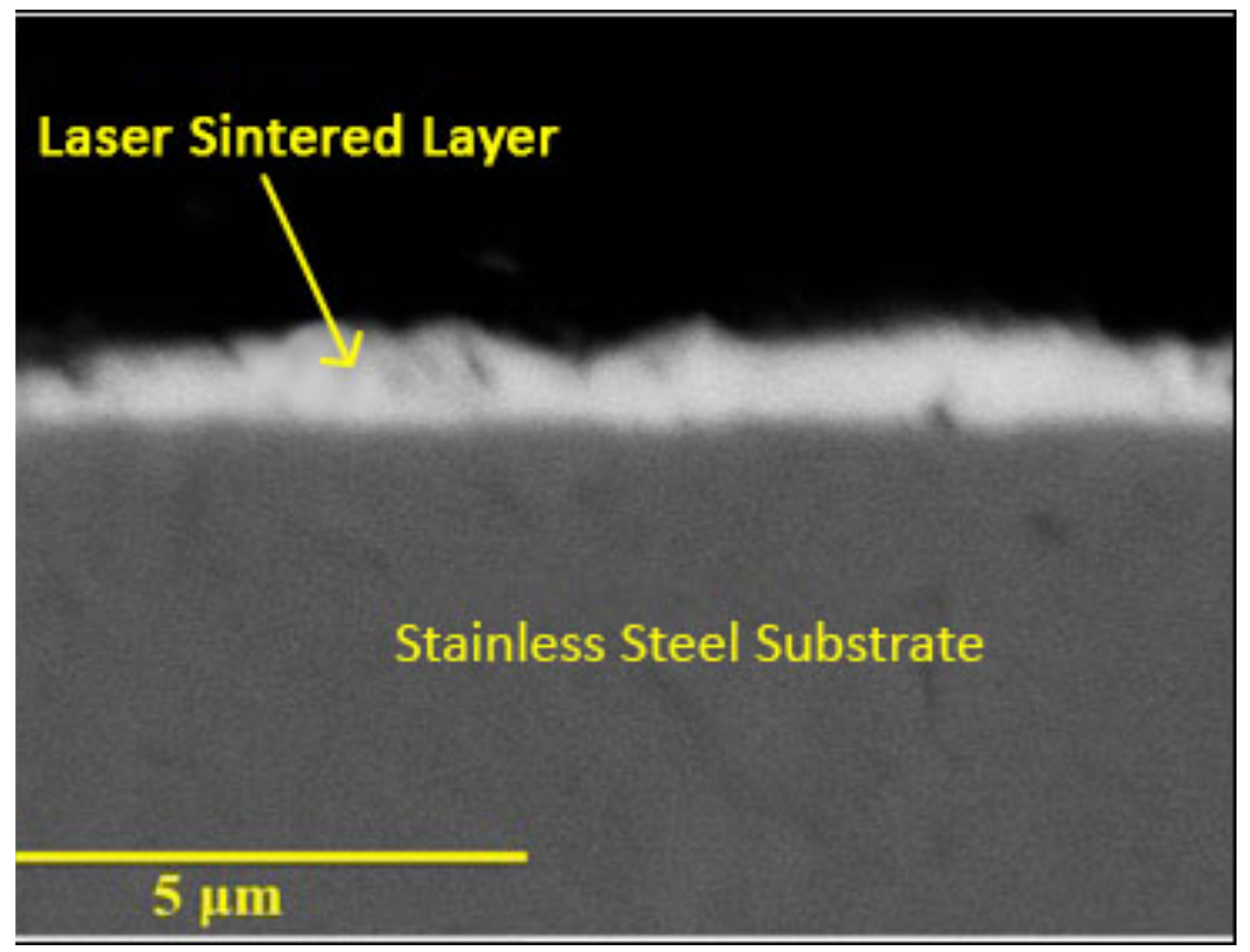

Fig 21. SEM image of cross section of sintered layer on stainless steel substrate. 
are make up the composition of SS and are shown in fig, $22 \mathrm{~b}$, c, and d respectively. There is barely any $\mathrm{Cr}$ or $\mathrm{Fe}$ in the sintered layer and some trace amount of $\mathrm{Ni}$ is present. Knowing that tungsten is the only major element present in the sintered layer, we can reason that the optical properties are due to the roughness of sintered tungsten surface.
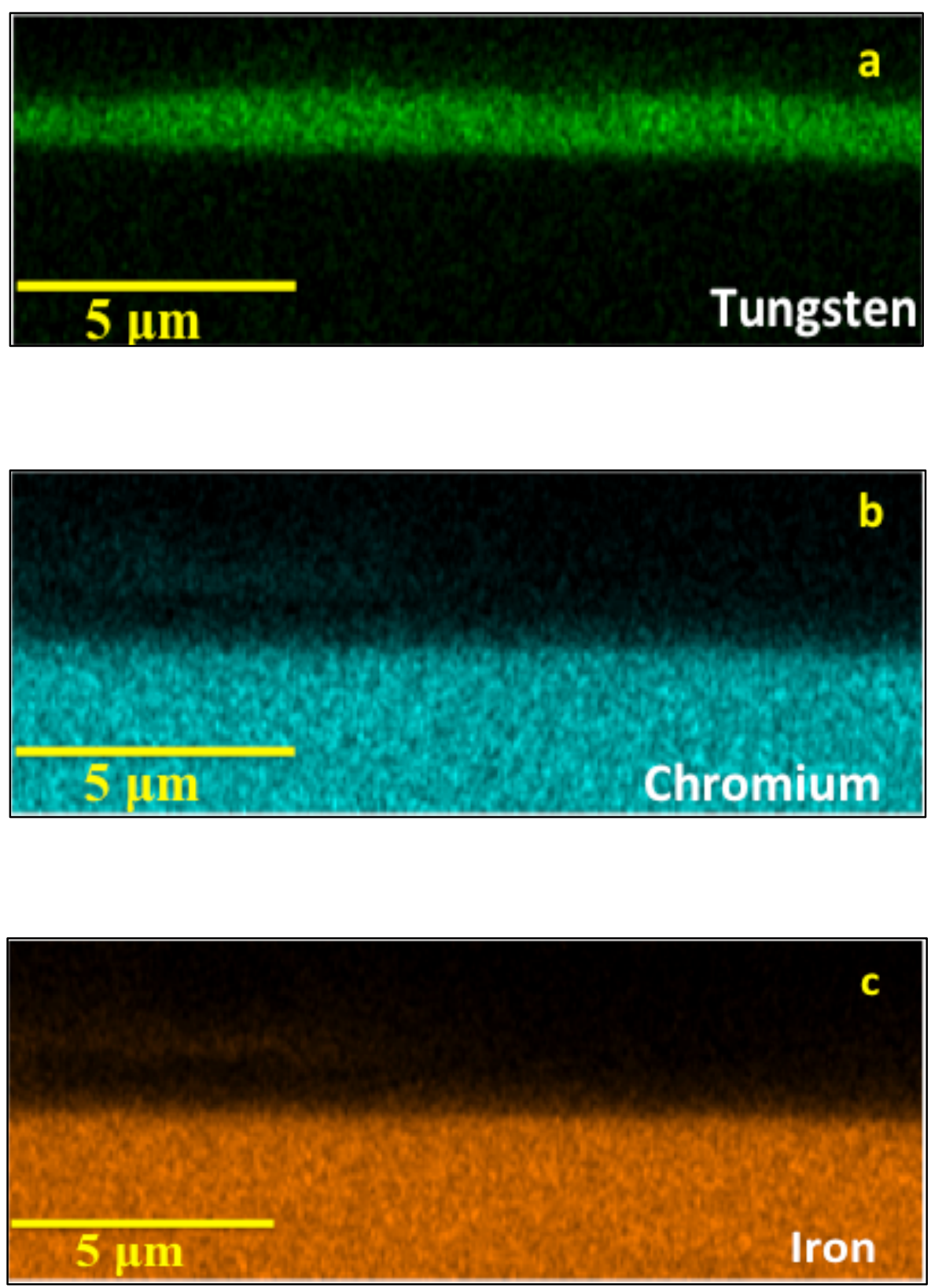


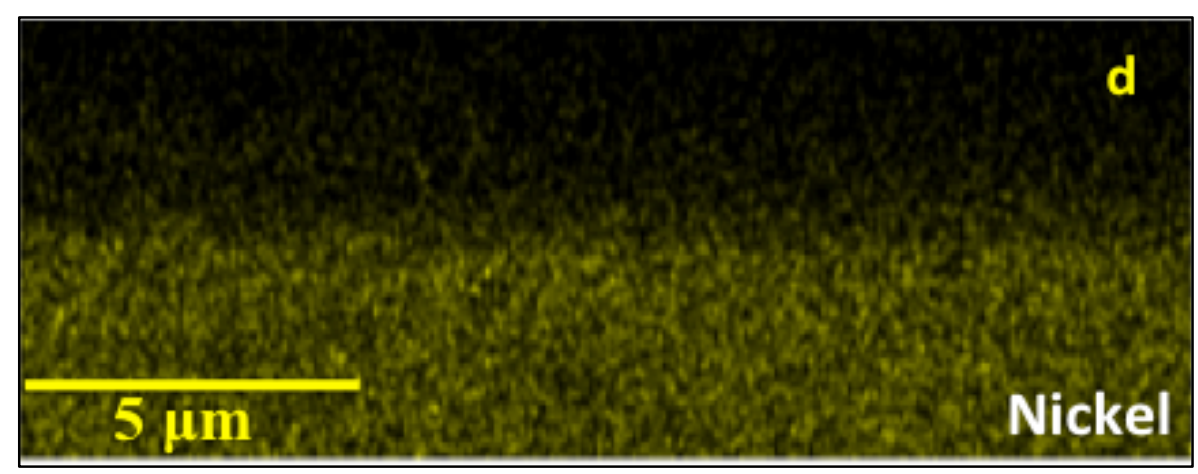

Fig 22. SEM images of the cross-section of sintered layer on stainless steel substrate showing elemental composition having (a) tungsten, (b) chrome, (c) iron, and (d) nickel.

\subsection{Structural characterization using $x$-ray diffraction (XRD)}

X-ray diffraction is a common technique to study crystal structures and atomic spacing. X-ray diffraction is based on constructive interference of monochromatic X-rays. These X-rays are produced by a cathode ray tube that are collimated and made to concentrate on the sample. The interaction of the incident rays with the sample produces constructive interference and a diffracted ray when conditions satisfy Bragg's Law. Braggs's Law relates the wavelength of electromagnetic radiation to the diffraction angle and the lattice spacing in a crystalline sample. By scanning the sample through a range of angles, all possible diffraction directions of the lattice are obtained. The diffraction peaks that are produced are converted to d-spacings that allow for identification of the material. A database is prepared for d-spacings of materials which is used for identification of the investigating material.

The instrument supplied by Rigaku Corporation was used for XRD analysis using a $\mathrm{CuK} \alpha$ radiation source. Figure 23 shows the XRD data of the sintered film after second scan. From X-ray measurement we observe ferberite $\left(\mathrm{FeO}_{4} \mathrm{~W}\right)$ phase peaks along with iron peaks from stainless steel as shown in fig. 23. The coating is $\sim 1 \mu \mathrm{m}$ thick so the X-rays can penetrate the 
coating and pick up the stainless steel (iron) signal. Nickel, chrome, and others in form of solid solution thus they don't show up in XRD. The ferberite phase is formed during the laser sintering process because very high temperatures are reached and so the tungsten and iron in presence of air form this compound. Iron oxide $\left(\mathrm{Fe}_{2} \mathrm{O}_{3}\right)$ peaks are also formed for the same reason as high temperatures are reached on stainless steel substrate. The peak positions were identified based on the reported x-ray diffraction data (Card No. 9000224 in Rigaku Corp. software database).

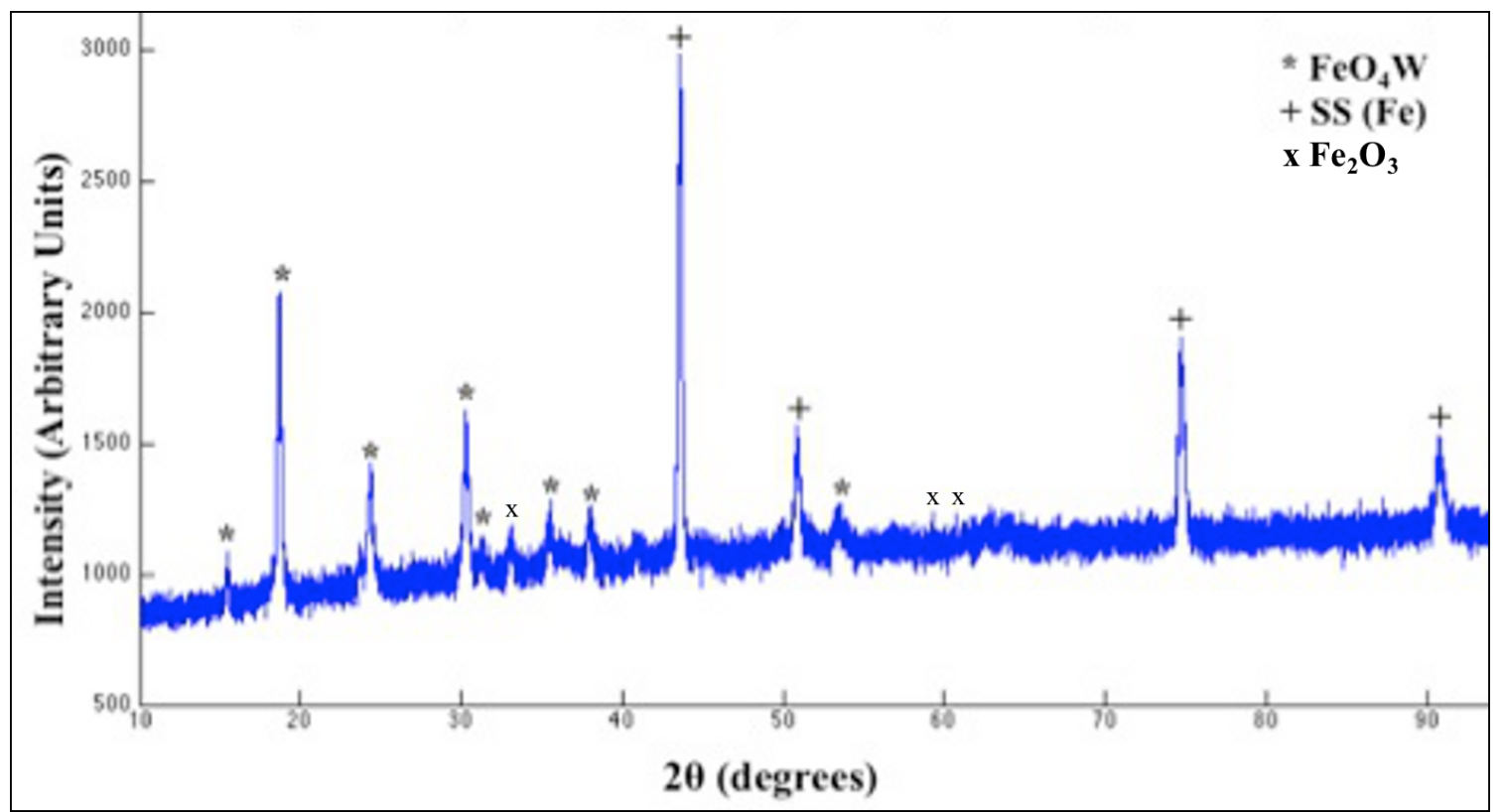

Fig 23. XRD spectra of the laser sintered tungsten coating after second scan. 


\section{Chapter 5. Optical and Infrared Properties of Laser}

\section{Sintered Tungsten Films}

\subsection{Optical absorption}

Solar absorptance measurements were done using an integrating sphere, supplied by Labsphere Inc., and laser wavelengths of $410 \mathrm{~nm}, 532 \mathrm{~nm}, 633 \mathrm{~nm}, 790 \mathrm{~nm}$, and $1064 \mathrm{~nm}$ were used. Solar absorptance of bulk tungsten metal was $\sim 48 \%$ which is in agreement with the literature data [13]. Below in table 5 are absorptance values at three different wavelengths. As can be seen, the absorptance is approximately constant at all wavelengths and thus an average of all the measurements was taken and represented as solar absorptance.

Table 5. Absorptance values at three different wavelengths

\begin{tabular}{|l|c|c|c|}
\hline & $\mathbf{5 3 2} \mathbf{n m}$ & $\mathbf{6 3 3} \mathbf{~ n m}$ & $\mathbf{1 0 6 4} \mathbf{~ n m}$ \\
\hline Sintered layer after $\mathbf{1}^{\text {st }} \mathbf{s c a n}$ & $67.8 \%$ & $73.1 \%$ & $70 \%$ \\
\hline Sintered layer after $\mathbf{2}^{\text {nd }}$ scan & $88.3 \%$ & $92.2 \%$ & $91 \%$ \\
\hline
\end{tabular}

Solar absorptance of $\sim 70 \%$ was achieved for sintered tungsten layer after the first laser scan with $20 \%$ laser power and $\sim 90.3 \%$ was achieved after scanning the sintered layer the second time with laser power of $40 \%$. This second scan, as can be seen, significantly increased $\alpha$ to $90.3 \%$ and this is due to the increase in surface roughness as seen from SEM in fig 18. The height and spacing of the random roughness of the sintered layer varies from a micron to at least 
a couple of microns. There is also sub-micron roughness on peaks and valleys that can contribute to increased solar absorption. This surface behaves as a graded index medium $[13,19]$ when the roughness is sub-wavelength thus leading to increased solar absorptance. When the roughness is larger than the wavelength, the increased absorptance is due to the light getting trapped by multiple reflections.

\subsection{Infrared reflectance}

The sample was illuminated by IR wavelengths ranging from 2.5 to 12.5 microns and the reflected IR light was captured to provide a reflectance versus wavelength data.

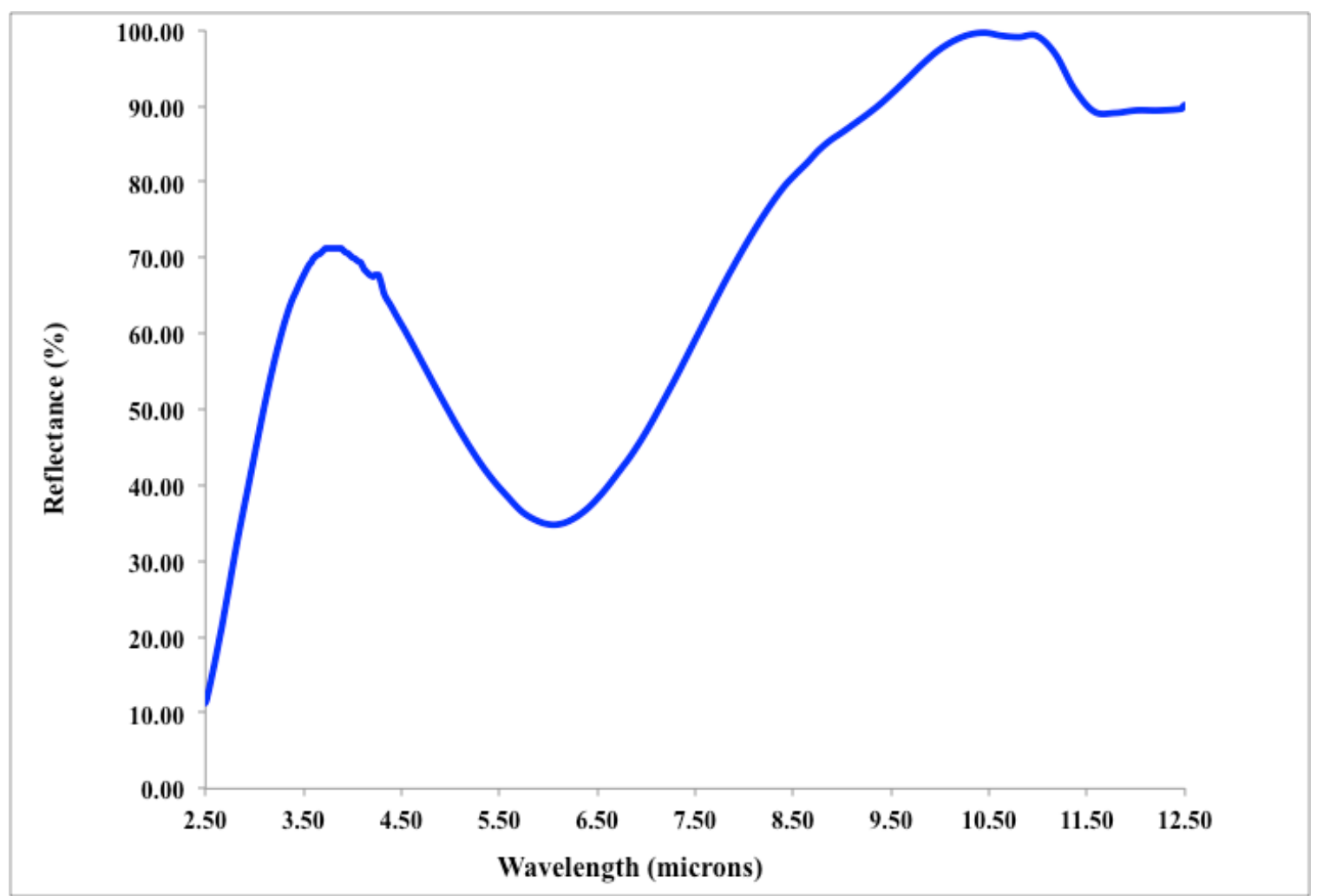

Fig 24. IR reflectance plot of the laser sintered coating before heat treatment. 
Figure 24 shows IR reflectance plot of the sintered layer after $2^{\text {nd }}$ scan. The shape of the reflectance curve is governed by the surface morphology of the sintered coating. There is a decrease in reflectance at $6 \mu \mathrm{m}$ wavelength that cannot be observed for bulk tungsten. In order to understand the origin of the decreased reflectance the effect of plasmons was studied.

Plasmons: Metals are predominantly reflective from spectral range of microwave and IR all the way up to visible part of the electromagnetic spectrum. At near-IR and visible spectrum the electromagnetic filed penetration increases leading to higher absorption. At ultraviolet frequencies, metals start allowing propagation of electromagnetic waves with varying degrees of attenuation depending on their electronic band structure [39]. The dispersive properties of metals depend on the complex dielectric function which leads to varying optical properties across the spectrum. Previous work [40][41] has shown that increased absorption in the near IR region of tungsten periodical gratings can take place due to surface plasmon polaritons (SPP) excitation. SPPs are local excitation of electromagnetic waves and they exist and propagate along the interface of two different media [41]. The energy of surface plasmons in metals usually exist near the ultraviolet range or visible range for some metals. Photons that have lower energy than surface plasmon get totally reflected from the metal surface. But visible and IR light can be coupled with surface plasmons by introducing surface grating on metallic substrates that periodically perturbs the electron density near the surface [40]. In general, materials must have negative real part of dielectric constant to support surface plasmons and tungsten meets this requirement. When the electromagnetic wave enters the grating surface it gets coupled with surface plasmon and is absorbed [40]. Localized SPPs can thus cause a sharp decrease in reflectance in near IR region and the magnitude and position of the dip can be controlled by changing grating parameters. Fig. 25 below is taken from [41] to demonstrate the enhanced 
absorption caused by SPPs in the near IR region. It shows the structure of 1D tungsten periodical grating that was used for calculations to see the effect of grating height on absorptance in visible and near IR region. Fig. 26 shows spectral performance of the grating and we observe that there is indeed enhanced absorption at $1.5 \mu \mathrm{m}, 2 \mu \mathrm{m}$, and $2.5 \mu \mathrm{m}$ for grating heights $200 \mathrm{~nm}, 300 \mathrm{~nm}$, and $400 \mathrm{~nm}$ respectively. Thus one can get enhanced absorption in the near IR region from tungsten gratings due to plasmonics that cannot be obtained if bulk tungsten is used. Similar results were observed in [40] where they used rectangular periodical 1D gratings and peaks between $1-2.5 \mu \mathrm{m}$ were observed which were due to SPP excitation.

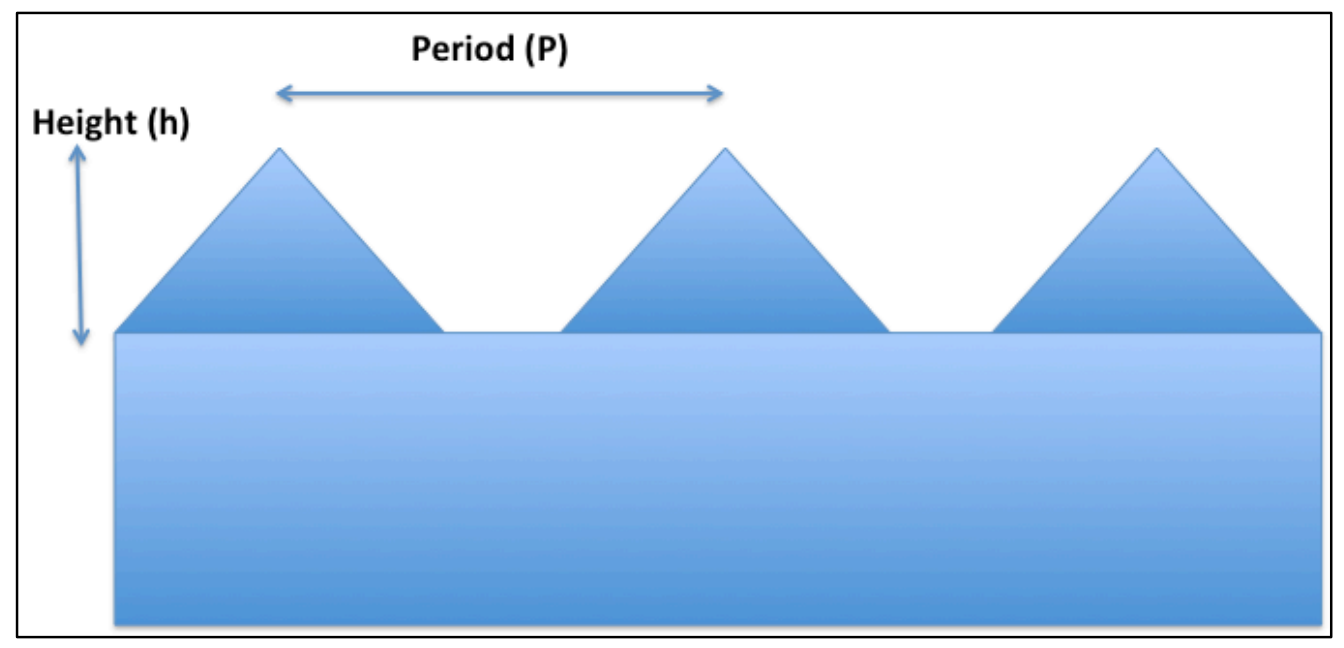

Fig 25. Tungsten 1D periodical grating.

We have observed increased absorption at $6 \mu \mathrm{m}$ which is much further away in the IR than was observed by [40] and [41]. And there are no other reports that show enhanced absorption at beyond $4 \mu \mathrm{m}$ for tungsten surface. This leads us to conclude that the dip in reflectance is due to the surface roughness and not because of any plasmonic interaction taking place at the surface. 


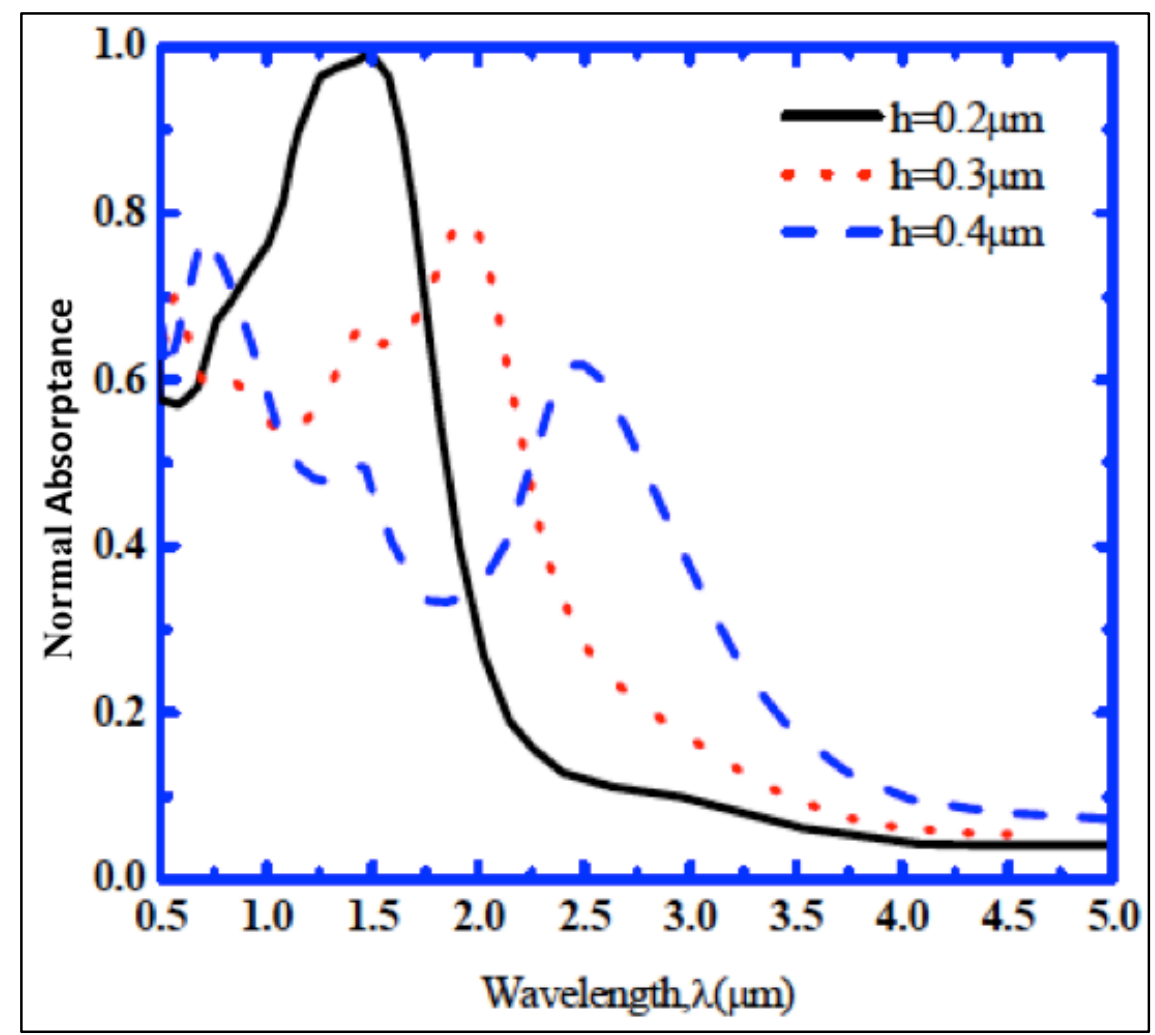

Fig 26. Normal absorptance plot of the tungsten grating with period $500 \mathrm{~nm}$ and varying heights [41].

Theoretical modeling performed on random roughness similar to sintered layer on tungsten surface also shows this dip and the position of the dip depends on the period of the roughness. Beyond wavelength of $6 \mu \mathrm{m}$, the reflectance rises and approaches $\sim 90 \%$, as the wavelength is getting much larger than the surface roughness.

\subsection{Effect of antireflection coating on optical and infrared properties}

We have deposited solution based $\mathrm{TiO}_{2}$ coatings on sintered films in order to further increase solar absorptance. $\mathrm{TiO}_{2}$ coatings were prepared using sol-gel technique. Titanium(IV) 
isopropoxide (97\%), acetylacetone (acac, 99\%) and i-PrOH (propanol) were purchased from Sigma-Aldrich. The titania solution was prepared by mixing titanium isopropoxide $(0.64 \mathrm{~mL})$, acac $(0.23 \mathrm{~mL})$, and i-PrOH $(6.4 \mathrm{~mL})$ and the mixture was stirred for one hour. Then the solution was spin coated on sintered coatings at different speeds ranging from $1000 \mathrm{rpm}$ (revolutions per minute) to $4000 \mathrm{rpm}$ to determine the optimal thickness and the films were baked for 5 minutes at $200{ }^{\circ} \mathrm{C}$. The coatings produced at 1000 and $2000 \mathrm{rpm}$ were thicker and they caused higher absorption in the IR thus these speeds were not chosen. Coatings produced with using 3000 and $4000 \mathrm{rpm}$ were optimal since they did not have significant absorption in the IR. Solar absorptance of sintered samples before and after $\mathrm{TiO}_{2}$ coating was $\sim 89 \%$ and $\sim 92.5 \%$ respectively. This shows an increase in solar absorptance that is highly desired. Fig. 27a and b show the IR reflectance plot of SS substrate and sintered coating before and after applying $\mathrm{TiO}_{2}$ coating. As it can be seen, the IR reflectance before and after applying the coating is very similar thus implying there is no effect of $\mathrm{TiO}_{2}$ on IR properties.

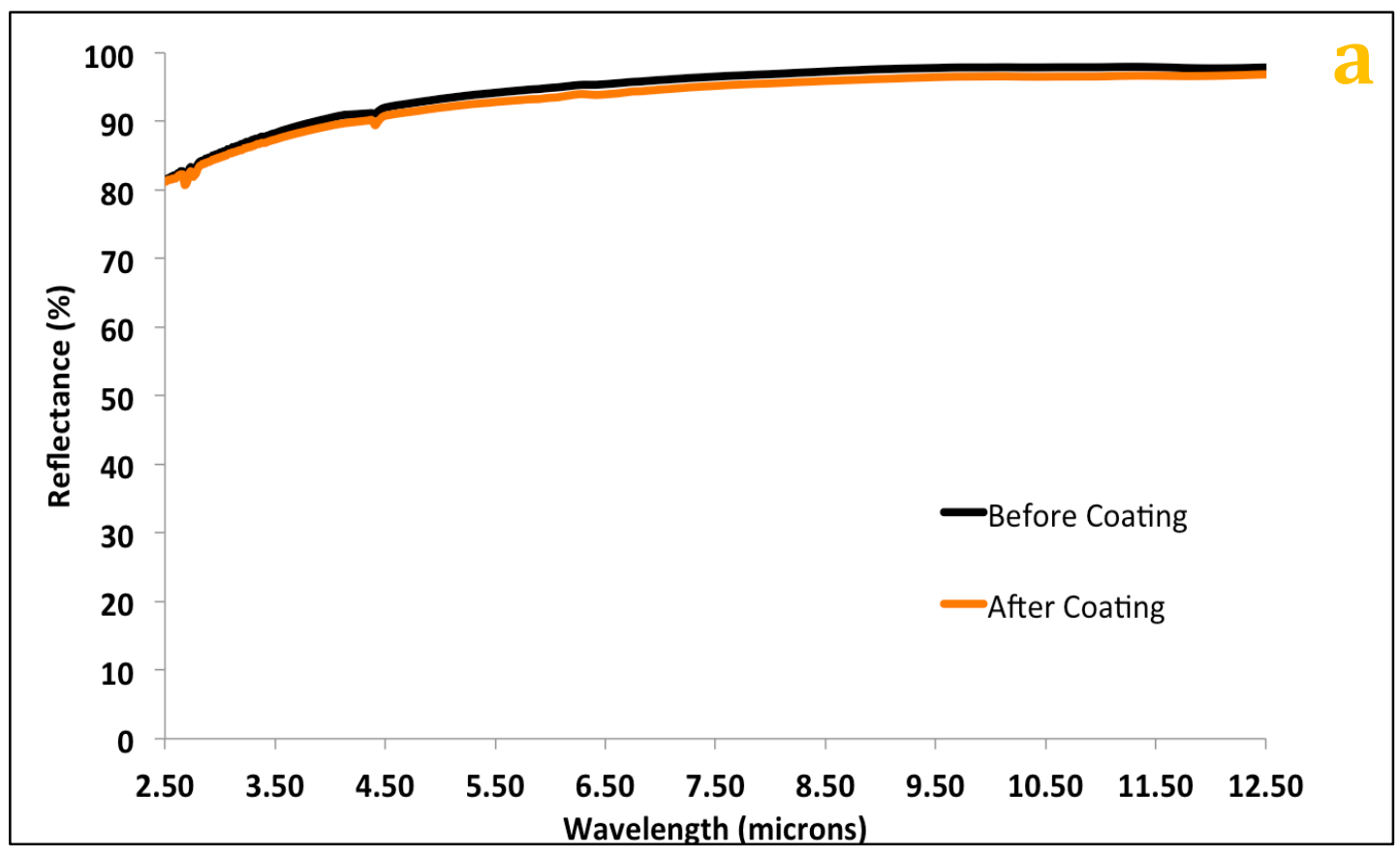




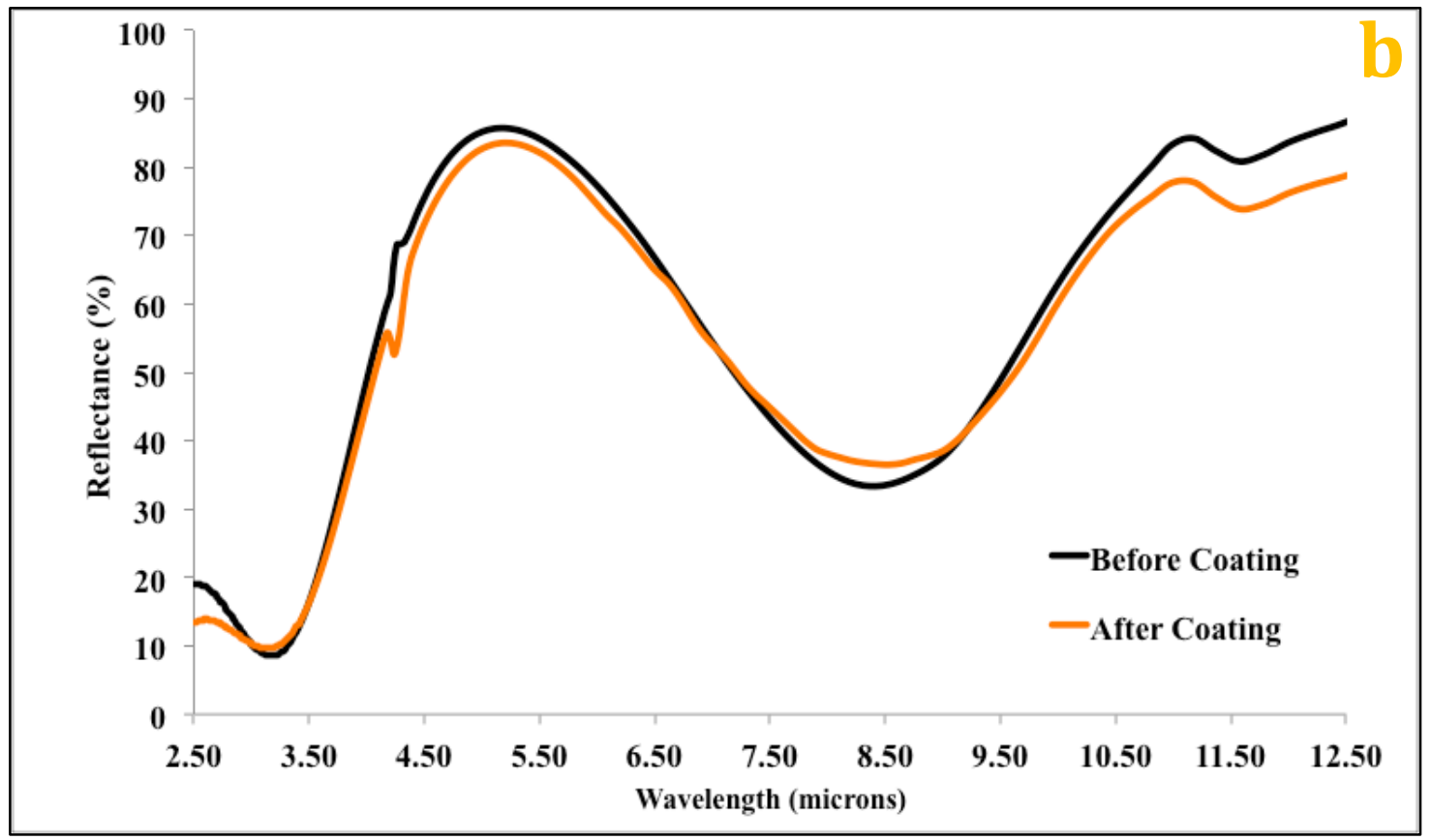

Fig 27. IR reflectance of (a) SS substrate and (b) sintered coatings before and after $\mathrm{TiO}_{2}$ coating. 


\section{Chapter 6. Thermal Stability of Laser Sintered Tungsten}

\section{Films}

\subsection{Effect of temperature on optical, infrared, and compositional Properties}

Stainless steel is thermally stable in presence of oxygen at higher temperatures and is one of the the reasons why it is used in solar thermal applications. There was barely any change in solar absorptance and IR reflectance of stainless steel substrate below $600{ }^{\circ} \mathrm{C}$ in air. After heat treatment at $600{ }^{\circ} \mathrm{C}$ for two hours change was observed in spectral data. The solar absorptance increased from $\sim 34 \%$ before heat treatment to $37 \%$ after heat treatment at $600{ }^{\circ} \mathrm{C}$. IR reflectance dropped by approximately $\sim 3-5 \%$ from 3.5 to 12.5 microns in wavelength as seen in figure $28 \mathrm{a}$. This tells us that the stainless steel substrate is thermally stable up until the operating range of solar thermal operation with only slight modifications. Tungsten on the other hand forms oxides of different compositions based on what temperature it is exposed to air. Based on our investigation, we have observed a slight increase in solar absorptance from $\sim 48 \%$ before heat treatment to $\sim 50 \%$ after heat treatment at $400{ }^{0} \mathrm{C}$ for 2 hours. IR reflectance has dropped by nearly $10 \%$ at 2.5 micron wavelength and then the drop decreases to reach an absolute drop of $\sim 3 \%$ from 4.5 to 12.5 micron wavelength as seen in figure $28 \mathrm{~b}$. This implies that tungsten has to be coated with a protective coating in order to maintain its spectral performance and thermally stability beyond $400{ }^{0} \mathrm{C}$. The protective coating not only has to allow for higher temperature operation, but also should not alter the optical and IR properties of the spectral selective surface.

Laser sintered tungsten samples were heated to $300{ }^{0} \mathrm{C}, 400{ }^{\circ} \mathrm{C}, 500{ }^{\circ} \mathrm{C}$, and $600{ }^{\circ} \mathrm{C}$ under atmospheric pressure in order to evaluate the long term stability. For heating up to $600{ }^{\circ} \mathrm{C}$ for 

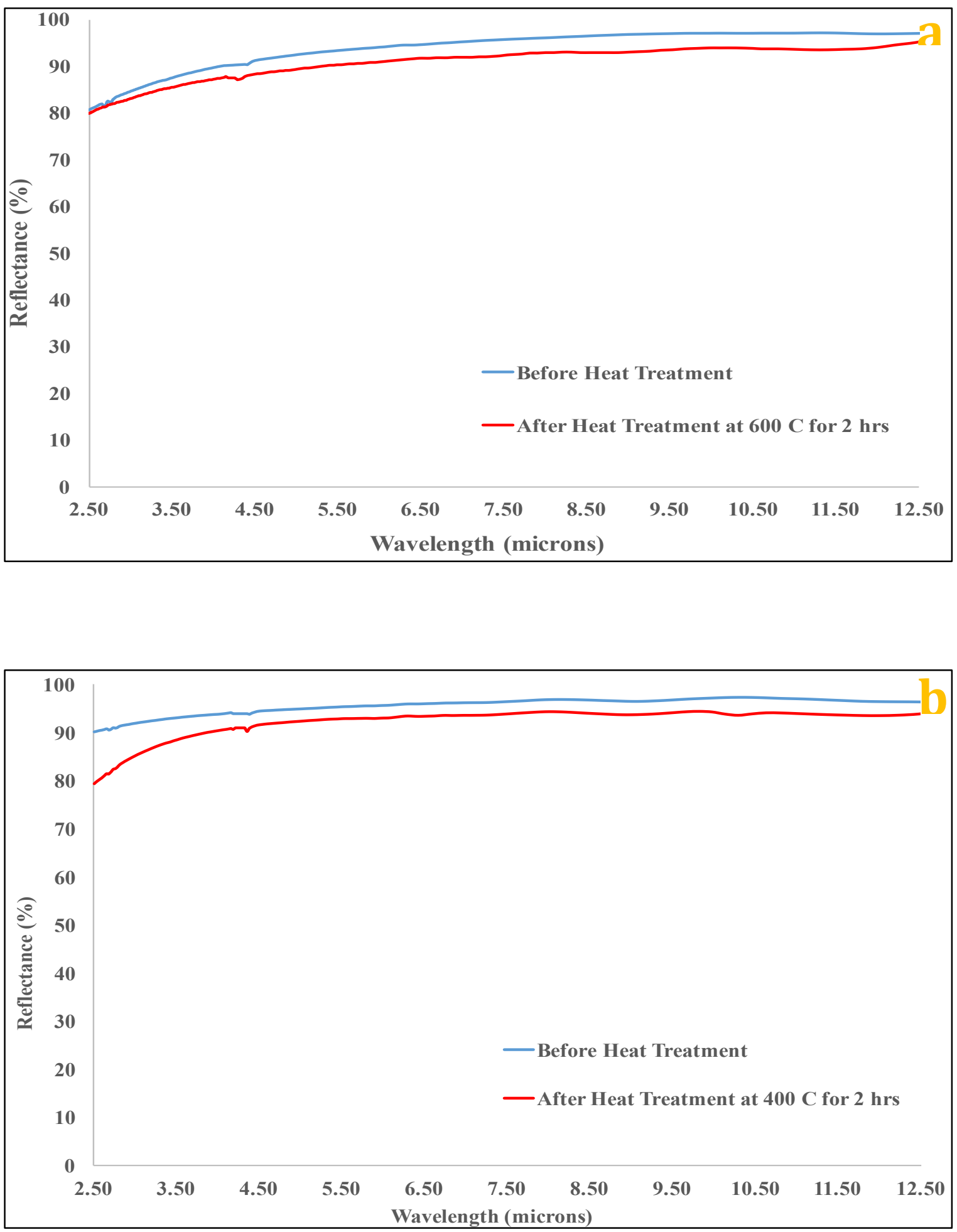

Fig 28. IR reflectance of (a) SS substrate before and after heat treatment at $600{ }^{\circ} \mathrm{C}$ for 2 hrs and (b) tungsten sample before and after heat treatment at $400{ }^{\circ} \mathrm{C}$ for 2 hrs. 
one hour no change in solar absorptance was observed. Since the commercial desired temperature operation range is $>600{ }^{0} \mathrm{C}$, the sintered coating was heat treated at $650{ }^{0} \mathrm{C}$ under atmospheric pressure for 2 hours, 12 hours, and then 36 hours. The results on the effect of heat treatment on solar absorptance are presented in table 6 .

Table 6. Solar absorptance of laser sintered coating before and after heat treatment

\begin{tabular}{|c|c|}
\hline & Solar Absorptance ( $\boldsymbol{\alpha})$ \\
\hline Room Temperature & $90.3 \%$ \\
\hline $\mathbf{6 5 0}{ }^{\mathbf{0}} \mathbf{C}$ for $\mathbf{2}$ hours & $87.0 \%$ \\
\hline $\mathbf{6 5 0}^{\mathbf{0}} \mathbf{C}$ for $\mathbf{1 2}$ hours & $87.2 \%$ \\
\hline $\mathbf{6 5 0}^{\mathbf{0}} \mathbf{C}$ for 36 hours & $87.0 \%$ \\
\hline
\end{tabular}

To understand the change in solar absorptance and the possible effect of oxidation under high temperature treatment, EDS was performed. The film composition data are presented in table 7:

Table 7. EDS data in weight $(\mathrm{wt} \%)$ percent before and after heat treatment at $650{ }^{0} \mathrm{C}$.

\begin{tabular}{|c|c|c|c|}
\hline Composition & Room Temperature & $\mathbf{6 5 0}{ }^{\mathbf{0}} \mathbf{C}$ for & $\mathbf{6 5 0}^{\mathbf{0}} \mathbf{C}$ for $\mathbf{3 6}$ \\
& & $\mathbf{2}$ hours & hours \\
\hline Tungsten & 28.42 & 27.95 & 21.40 \\
\hline Iron & 38.66 & 35.78 & 42.30 \\
\hline Chromium & 9.21 & 8.83 & 8.30 \\
\hline
\end{tabular}




\begin{tabular}{|c|c|c|c|}
\hline Nickel & 3.19 & 2.74 & 2.40 \\
\hline Oxygen & 20.52 & 24.70 & 25.60 \\
\hline
\end{tabular}

The $w t \%$ of iron has increased after heat treatment for 36 hours and that could just be due to variation in composition at different locations and, as mentioned earlier, the equipment also has a $\sim 5 \%$ variation in the values it measures. It could also be that the iron may be diffusing out from within the coating and therefore tungsten is being picked up less by the EDS. Also, high temperatures are generated on the surface of the sample where laser sintering takes place. Since all this is done under atmospheric pressure, surface oxide could be formed on the coatings. This surface oxide acts as a protection layer that prevents further oxidation of sintered coatings when being heated in air at $650{ }^{\circ} \mathrm{C}$. Authors in [42] have laser textured titanium surface in air and they present a similar observation where the optical properties of the surface remains unchanged before and after heat treatment in air at $500{ }^{\circ} \mathrm{C}$. The surface oxide serves as a protection layer and also as an index-matching layer at tungsten-air interface that reduces front surface reflection, thereby contributing to higher solar absorptance. After heat treatment for 2 hours, there is some change in the surface morphology of the sintered coating as seen in fig. 29 when compared to fig. 20. This slight change in surface morphology of the laser sintered coating could have led to the decrease in $\alpha$. Authors in [13] fabricated sub-micron cylindrical cavities in bulk tungsten and achieved $\alpha \sim 82 \%$ after annealing at $1200 \mathrm{~K}$, however, heat treatment was done in vacuum. Authors in [34] fabricated dendritic textures on tungsten surface and were able to achieve $\alpha \sim$ 98.5 after heat treatment in air only up to $275{ }^{\circ} \mathrm{C}$ but a degradation in solar absorptance was observed at higher temperatures. This work presents first experimental result for achieving $\alpha \sim$ 


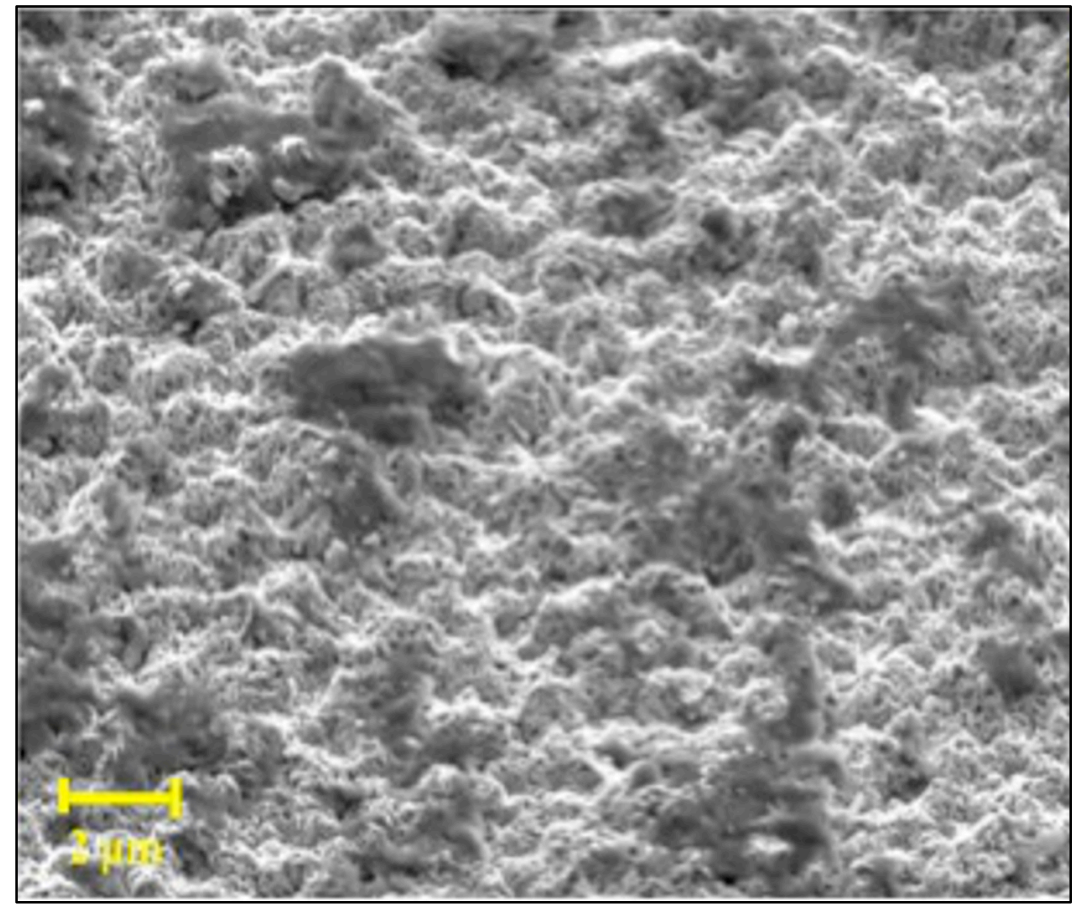

Fig 29. SEM image of laser sintered tungsten after heat treatment at $650{ }^{\circ} \mathrm{C}$ for two hours.

$87 \%$ after heat treatment at $650{ }^{\circ} \mathrm{C}$ for 36 hours in air for a spectral selective coating developed using tungsten.

Figure $30 \mathrm{a}$ and $\mathrm{b}$ show experimental and simulation of IR reflectance for sintered coating before and after heat treatment respectively. As seen in fig. 30a, there is a shift in the IR curve after heat treatment for two hours but the overall shape is still similar. After further heat treatment, there are only small shifts in the IR curve when the sintered coating is heat treated for 12 hours and then for 36 hours. To understand the shift in the IR peak from 3.7 to 4.7 microns after heat treatment, theoretical simulation of reflectance of the sintered surface before and after heat treatment was performed [43]. The simulation was carried out using finite difference time domain (FDTD) method [44] and the program was MIT electromagnetic equation propagation (MEEP) [45]. The surface dimensions were obtained from the SEM images shown in fig. 20. The 
features sizes were extracted using a software imaging technique where different heights and periods in an image can be represented by different colors. Those feature sizes obtained were then imported to MEEP and the simulation was carried out as explained in the modeling section (chapter 2). Figure 30b shows IR reflectance spectra of the simulation performed for sintered surface before heat treatment and after heat treatment for 2 hours. Changing the period slightly in the simulation of sub-micron surface roughness from $30 \mathrm{~nm}$ to $20 \mathrm{~nm}$ causes the shift in the peak. The periodicity of sub-micron features on sintered surface decreases by $\sim<50 \mathrm{~nm}$ after heat treatment since it gets a little smoother as seen in the SEM image. The SEM images are not that high in resolutions and so the program cannot approximate the exact surface. The purpose of the simulation was to explain the shift and not the magnitude of the reflectance values. Also, the initial shift in the IR reflectance after heat treatment for 2 hours was large but further heat treatments for longer duration produced only small changes. This can be due to the fact that once some oxide layer is formed, it is harder to grow further.

Fig. 31a shows XRD spectra of laser sintered coating before and after heat treatment at $650{ }^{\circ} \mathrm{C}$ for 2 hours and $31 . \mathrm{b}$ shows the XRD spectra after heat treatment at $650{ }^{\circ} \mathrm{C}$ for 36 hours. There is less noise in fig. $31 \mathrm{~b}$ as compared to fig. $31 \mathrm{a}$ and that is because a different equipment was used that pre-filtered the baseline and did some noise correction. However, the quality of data is not compromised and all the detectable peaks are present for analysis. The coating is $\sim 1$ $\mu \mathrm{m}$ thick so the $\mathrm{X}$-rays can penetrate the coating and pick-up the stainless steel (iron) signal. This is the first observation of ferberite formation using laser sintering method. It has been reported that ferberite is stable upto $950{ }^{\circ} \mathrm{C}$ in air [46] and that explains the thermal stability of laser sintered coating in addition to the protective surface oxide being present. In fig. 31a and b peaks 

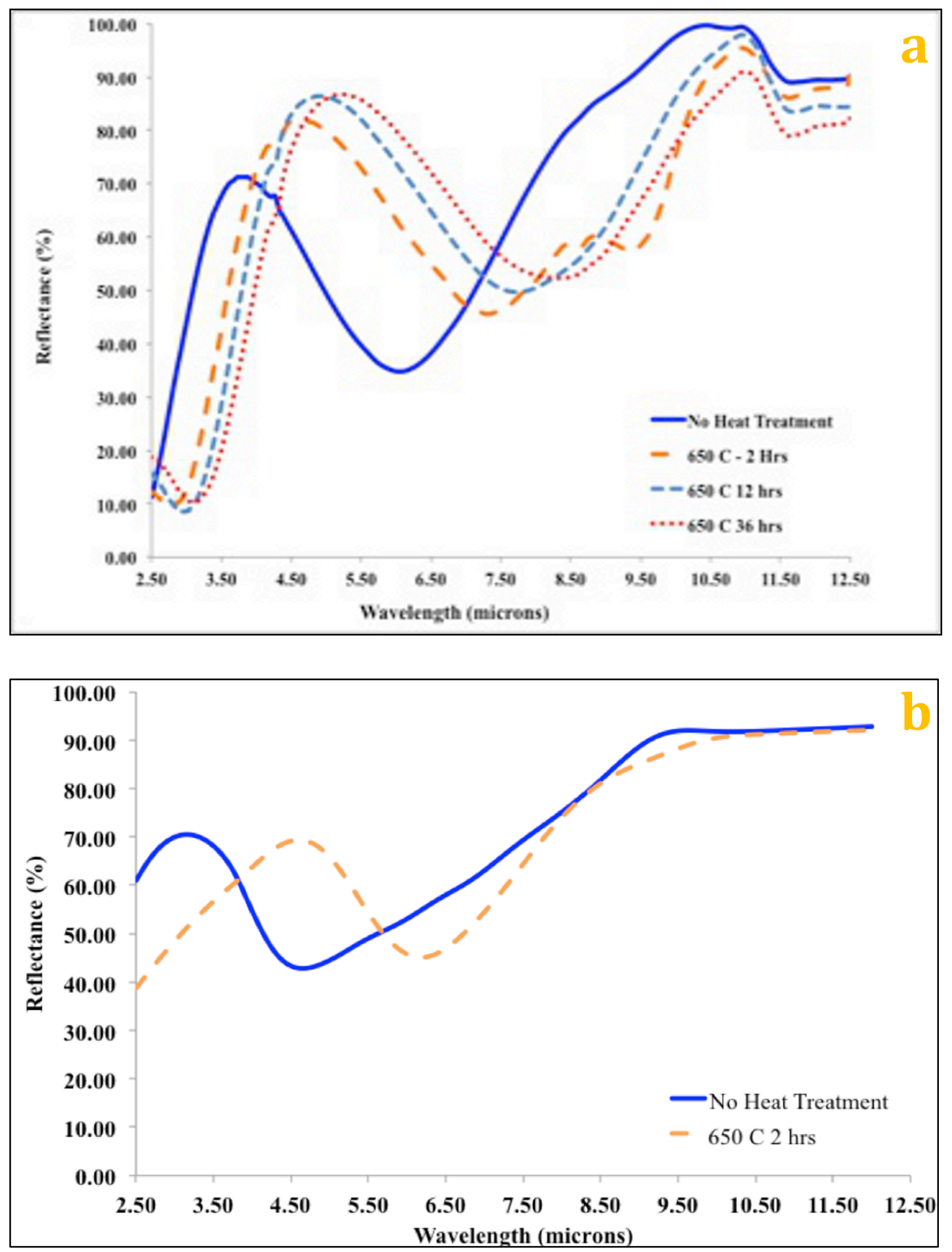

Fig 30. (a) IR reflectance of the laser sintered coating before and after heat treatment at $650{ }^{\circ} \mathrm{C}$ and (b) simulated IR reflectance before and after heat treatment at $650{ }^{\circ} \mathrm{C}$ for two hours. 

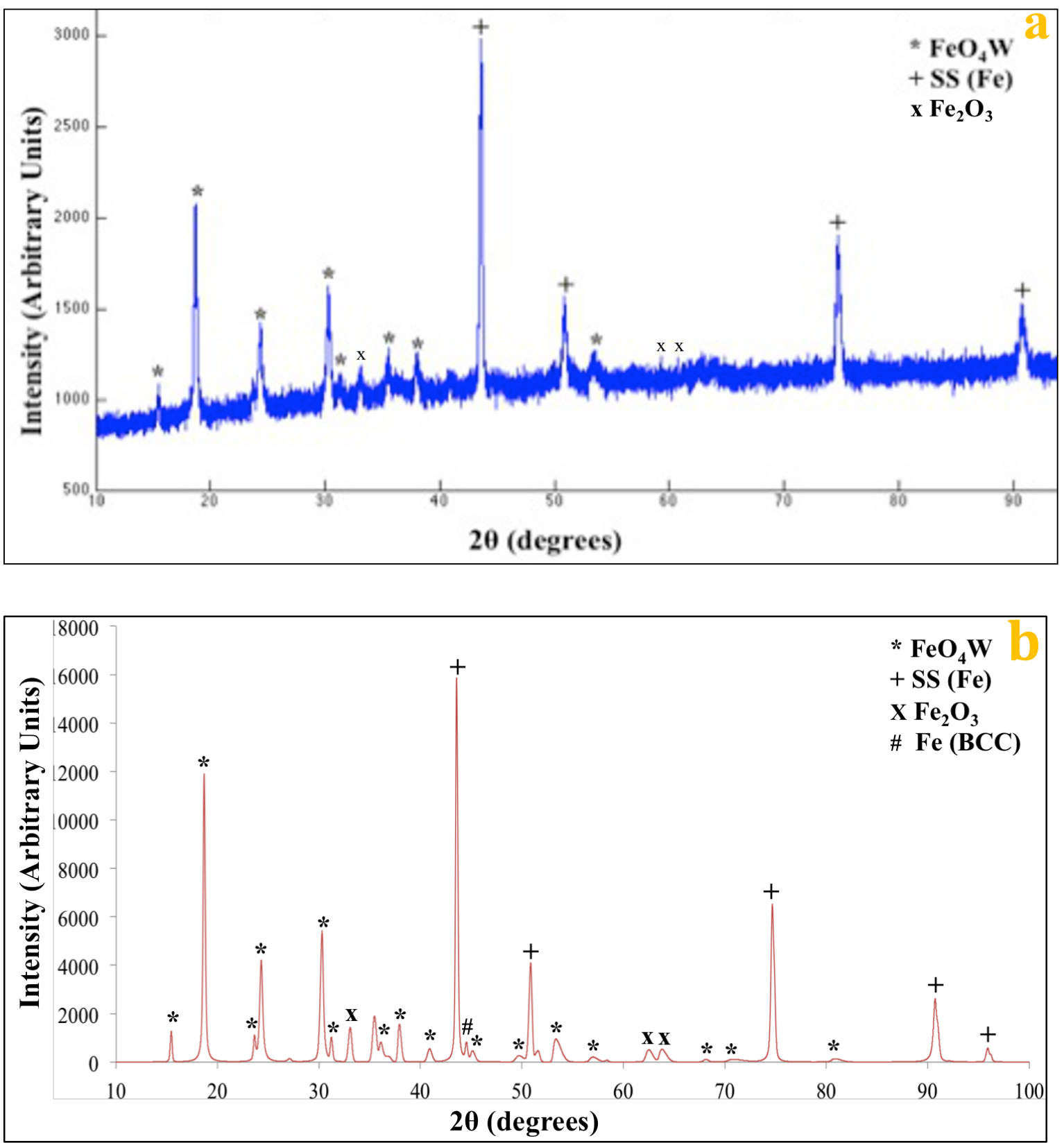

Fig 31. (a) XRD spectra of laser sintered coating after heat treatment at $650{ }^{\circ} \mathrm{C}$ for 2 hours and (b) after 36 hours.

of iron oxide iron are present and since they were there even before heat treatment (fig. 23), we can conclude that $\mathrm{Fe}_{2} \mathrm{O}_{3}$ was formed due to laser sintering taking place on SS substrate. Additional peak of body centered cubic (BCC) iron is present as seen in fig 31.b after being heat 
treated to $650{ }^{\circ} \mathrm{C}$ for 36 hours. This BCC iron peak present after 36 hours of heat treatment was also present when only a SS substrate was heated to $650{ }^{\circ} \mathrm{C}$ for 36 hours. This suggests that BCC iron peak occurs due to the substrate being heat treated. In cases involving heat treatment of materials at such high temperatures diffusion of elements from the interface of two media or also from within the substrate can take place. Authors in [47] report diffusion of elements from the substrate at high temperatures. In order to prevent this, a pre heat-treatment of SS in air at lower temperature can be carried out to form an oxide layer that will act as a barrier for diffusion.

\subsection{Effect of Laser Sintering Environment}

a. Sintering in nitrogen environment: Laser sintering of tungsten nanoparticles was carried out in nitrogen environment to study its effect on optical and infrared properties before and after heat treatment in vaccum and in air. Table 8 shows solar absorptance of one of the sintered coatings before and after heat treatment at $600{ }^{0} \mathrm{C}$ in vacuum and in air for two hours. The variation from location to location in solar absorptance is $+/-1-2 \%$. This shows that the sintered coating maintains its solar absorptance when heat-treated in vacuum but drops when

Table 8. Solar absorptance of sintered coating prepared under nitrogen atmosphere

before and after heat treatment at $600{ }^{\circ} \mathrm{C}$ for 2 hours

\begin{tabular}{|c|c|c|c|}
\hline $\begin{array}{c}\text { Solar } \\
\text { Absorptance }\end{array}$ & $\begin{array}{c}\text { Before Heat } \\
\text { Treament }\end{array}$ & $\begin{array}{c}\text { After Heat Treatment } \\
\text { in Vacuum }\end{array}$ & $\begin{array}{c}\text { After Heat Treatment } \\
\text { in Air }\end{array}$ \\
\hline $\boldsymbol{\alpha}$ & $\sim 70 \%$ & $\sim 68-70 \%$ & $\sim 60 \%$ \\
\hline
\end{tabular}


heat treated in air. Surface roughness will reduce due to heat treatment in general, but this significant drop is also due to the coating getting oxidized that further reduces surface roughness. We observed that the IR curve of the coating when heat-treated in vacuum stays similar to the curve before heat treatment. The SS substrate was also unaffected. However, when heat-treated in air, the IR curve drops for sintered coating and SS implying having a higher emittance, thus having poor thermal stability. Since laser sintering was carried out in nitrogen atmosphere, the sintered coating did not have a protective oxide layer to prevent further oxidation nor there was any formation of thermally stable compound like ferberite as observed in optimized coatings. Thus we concluded that laser sintering in presence of nitrogen environment was not suitable for achieving thermally stable coating. Thus sintering in nitrogen atmosphere was not pursued further.

b. Sintering Under Atmospheric Air: As we have previously seen that sintering under atmospheric air allows a surface oxide to form that prevents major oxidation. This is crucial for maintaining similar performance in optical and IR region of the spectrum before and after heat treatment. In table 9 , we see that solar absorptance does not change after being heat treated in vacuum or in air.

Table 9. Solar absorptance of sintered coating prepared under atmospheric air before and after heat treatment at $600{ }^{\circ} \mathrm{C}$ for 2 hours

\begin{tabular}{|c|c|c|c|}
\hline $\begin{array}{c}\text { Solar } \\
\text { Absorptance }\end{array}$ & $\begin{array}{c}\text { Before Heat } \\
\text { Treament }\end{array}$ & $\begin{array}{c}\text { After Heat Treatment } \\
\text { in Vacuum }\end{array}$ & $\begin{array}{c}\text { After Heat } \\
\text { Treatment in Air }\end{array}$ \\
\hline $\boldsymbol{\alpha}$ & $\sim 70 \%$ & $\sim 68-70 \%$ & $\sim 68-70 \%$ \\
\hline
\end{tabular}


IR reflectance measurements of the sintered coating heated at $600{ }^{0} \mathrm{C}$ in vacuum and in air were also conducted and we observed that the performance obtained before and after heat treatment was similar and therefore we concluded that it was thermally stable. Thus all our sintering was carried out in air as having that surface oxide is important to maintain high spectral selectivity and thermal stability. 


\section{Chapter 7. Applications of laser sintered coatings for solar}

\section{thermal and other areas}

\subsection{Solar tower systems}

The laser sintered coating would be applicable for solar central tower systems because it can maintain its performance and meet the desired operating temperature of $>600{ }^{\circ} \mathrm{C}$ and can be operated in air. As mentioned earlier, this high temperature operation will enable to meet the Department of Energy (DOE) goal of $\$ 0.06 / \mathrm{kWh}$. Multilayer cermets have been tried to be used as solar selective coatings [9 - 13] [15] [17] but their performance degrades in air due to oxidation at high temperatures. This high temperature operation also causes other problems for the coatings such as diffusion of metal particles, delamination of coatings, and cracking. Cermet coatings have been stable only up to $500{ }^{0} \mathrm{C}$ in air [47], which is far short of the desired temperature operation range. Currently, Pyromark 2500 series paint is the standard for central tower receivers that has $\alpha \sim 0.96$ and $\varepsilon \sim 0.86$. They suffer from high thermal losses [48] and the paint also degrades over time leading to lower performance and added cost to CSP systems [48]

Since central tower systems have high concentration factor, $\sim 600$, it is highly important to maximize solar absorptance. This can be seen by evaluating selective absorber efficiency as calculated using equation 4 :

$$
\eta=\frac{\alpha C I-\varepsilon \sigma T^{4}}{C I}
$$


where $\alpha, \varepsilon, \mathrm{I}, \mathrm{C}$, T stands for solar absorptance, thermal emittance, solar irradiance, concentration factor, and temperature respectively. If the solar absorptance is dropped from $87 \%$ to $80 \%$ for an emittance of $\sim 50 \%$ at $650{ }^{0} \mathrm{C}$, the selective absorber efficiency drops from $83.5 \%$ to $76.5 \%$. This is a significant drop in absorber efficiency, therefore achieving higher solar absorptance is very important. This does not mean that controlling emittance is not important, but it does not have as much an effect on absorber efficiency. After an optimum solar absorptance is reached, controlling the emittance can benefit further to improve the overall selective absorber efficiency as can be seen in the table 10 .

Table 10. Selective absorber efficiency with solar absorptance of $87 \%$.

\begin{tabular}{|c|c|c|c|}
\hline \multirow{2}{*}{ Emittance } & \multicolumn{3}{|c|}{ Efficiency at Various Temperatures } \\
\cline { 2 - 4 } & $\mathbf{6 5 0}{ }^{\mathbf{0}} \mathbf{C}$ & $\mathbf{7 0 0}^{\mathbf{0}} \mathbf{C}$ & $\mathbf{7 5 0}^{\mathbf{0}} \mathbf{C}$ \\
\hline $\mathbf{0 . 4 9}$ & 83.6 & 82.9 & 81.9 \\
\hline $\mathbf{0 . 7 0}$ & 82.2 & 81.1 & 79.8 \\
\hline $\mathbf{0 . 9 0}$ & 80.8 & 79.4 & 77.7 \\
\hline
\end{tabular}

We can see for current laser sintered coatings at $650{ }^{\circ} \mathrm{C}$ that the selective absorber efficiency is $83.6 \%$ but if the emittance was at 0.9 , the efficiency would drop to $80.8 \%$. The concern becomes even greater at higher temperatures where the drop in efficiency is larger, for example at $750{ }^{0} \mathrm{C}$, the efficiency at emittance of 0.49 is $81.9 \%$ and for emittance of 0.9 is only $77.7 \%$. To the best of our knowledge, this is the first experimental demonstration of achieving $\alpha \sim 87 \%$ after heat treatment at $650{ }^{0} \mathrm{C}$ for 36 hours in air. Therefore, this makes the coating very attractive for 
tower systems since receiver coatings that are capable of operating in air at high temperatures are highly desirable.

\subsection{Radiative/High Emittane Coatings}

Laser sintered coatings can be fabricated to either have low emittance, as in the case for solar thermal, or have high emittance to enhance radiation. Stainless steel is used in high temperature environments as heat exchangers for various applications. The goal is to conduct heat from the hot side and reject heat to the other side of the metal as radiation. However, polished metals have very low emittance and therefore need to be coated with materials that can withstand high temperatures, be oxidation resistant, and can act as radiators. Thus, laser sintering of tungsten can be performed to develop a coating that has low reflectance in the IR for it to have high emittance. A scan speed of $1 \mathrm{~mm} / \mathrm{s}$ and higher laser power, $\sim 50-60 \%$, was used to form sintered coating. As mentioned in section 4, larger surface roughness was formed at higher laser powers. This roughness was $>5 \mu \mathrm{m}$ and therefore led to higher absorptance in IR. This caused coatings to have higher emittance, which is required for radiation applications. Fig. 32 shows one such example of the laser sintered coating that has low reflectance in the IR and thus will have higher emittance. 


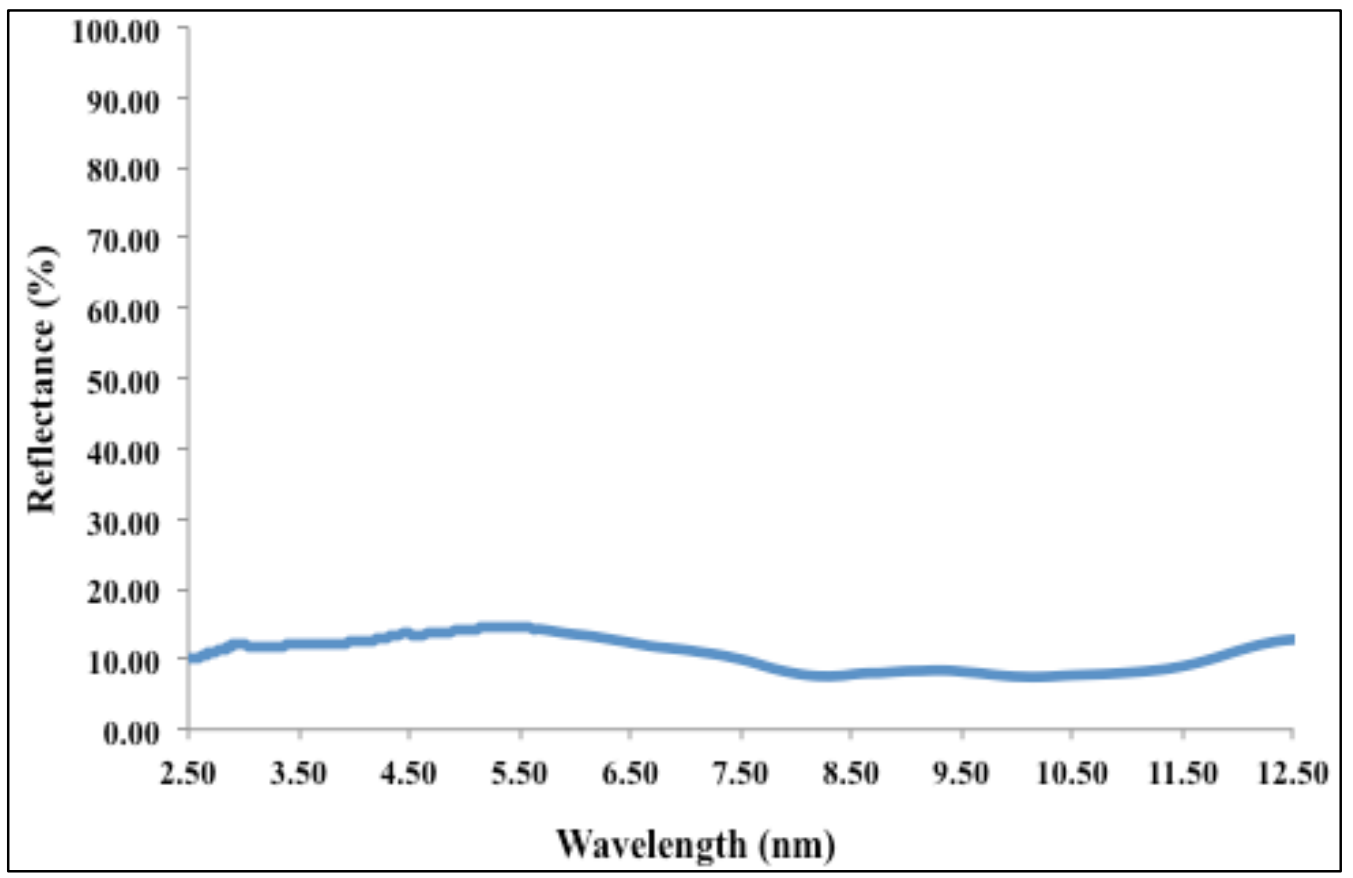

Fig 32. IR reflectance plot of laser sintered coating for high radiation application prepared under $60 \%$ laser power. 


\section{Chapter 8. Additional Research}

\subsection{Glancing angle deposition (GLAD) of tungsten nanostructures}

A collaboration with Oak Ridge National Laboratory (Dr. Ivan Kravchenko) was established for the fabrication of glancing angle deposited tungsten film on stainless steel substrate [49]. Also, graduate student Craig Ungaro in our group in collaboration with Argonne National Laboratory performed the modeling of optical absorption and infrared reflectance properties of GLAD films.

Figure 33 shows SEM image of tungsten structures on SS substrate deposited by GLAD. Tungsten nanostructured films were obtained by means of GLAD technique. The substrate tilt angle was kept at 85 degrees and the sample rotation speed was $20 \mathrm{rpm}$. Films were obtained by electron beam evaporation. The evaporator chamber vacuum was kept at $4 \times 10^{-3} \mathrm{~Pa}$. The structures are randomly arranged and the height of the structures varies from $\sim 130-202 \mathrm{~nm}$ as seen in fig. 33. The width of the structures varies from $\sim 40-100 \mathrm{~nm}$ and the spacing between them varies from $\sim 10-50 \mathrm{~nm}$. These tungsten structures were coated with $\mathrm{HfO}_{2}$ to act as an antireflection layer and enhance stability. Thus the graded index behavior in combination with antireflection effect led to solar absorptance $\sim 87.4 \%$. The structures were thermally stable as after heat treatment at $500{ }^{0} \mathrm{C}$ for 1 hour, the solar absorptance was $\sim 88.1 \%$. This indicated the importance of $\mathrm{HfO}_{2}$ coating as it also served as a protective layer during heat treatment. 


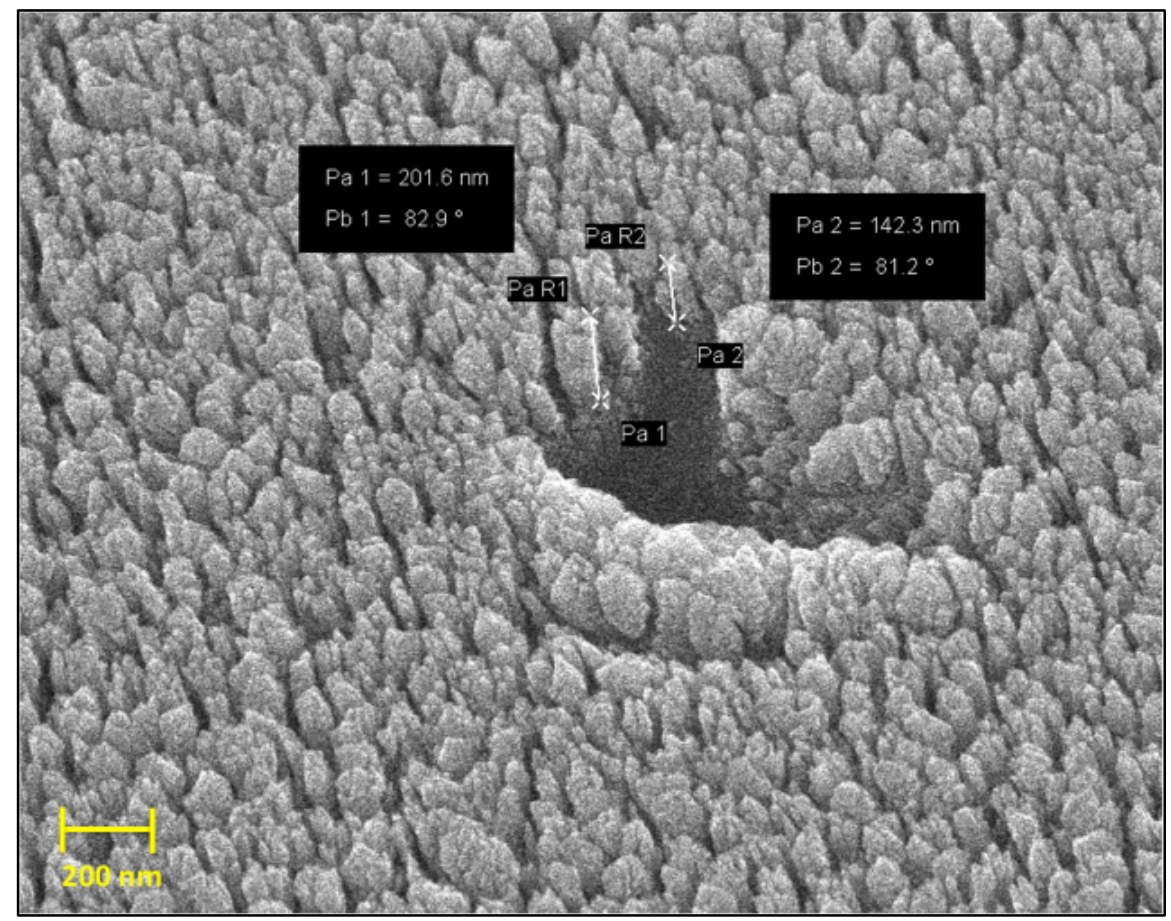

Fig 33. SEM image of GLAD tungsten structures on SS substrate (provided by ORNL).

Fig. 34 shows IR reflectance plot of the W structures with $\mathrm{HfO}_{2}$ coating before and after heat treatment. The shape of the curve is governed by surface morphology of the tungsten structures. The dip in the curve between 4 and 6 microns can be attributed to occur due to surface structure effects. Beyond wavelength of 5.5 microns, the reflectance starts rising and approaching to that of bulk tungsten as the structure dimensions are much smaller than the wavelength. After heat treatment at $400{ }^{0} \mathrm{C}$, the IR reflectance remains unchanged indicating thermal stability of the coating in air. A slight shift to the right can be seen in the IR reflectance after heating the sample at $500{ }^{0} \mathrm{C}$ in air and this could be due to interference effect from slight oxidation or a surface morphology change during heat treatment. However, the structures still maintain their performance as beyond 6.5 microns the reflectance approaches that of bulk tungsten and also the solar absorptance remains unchanged. 


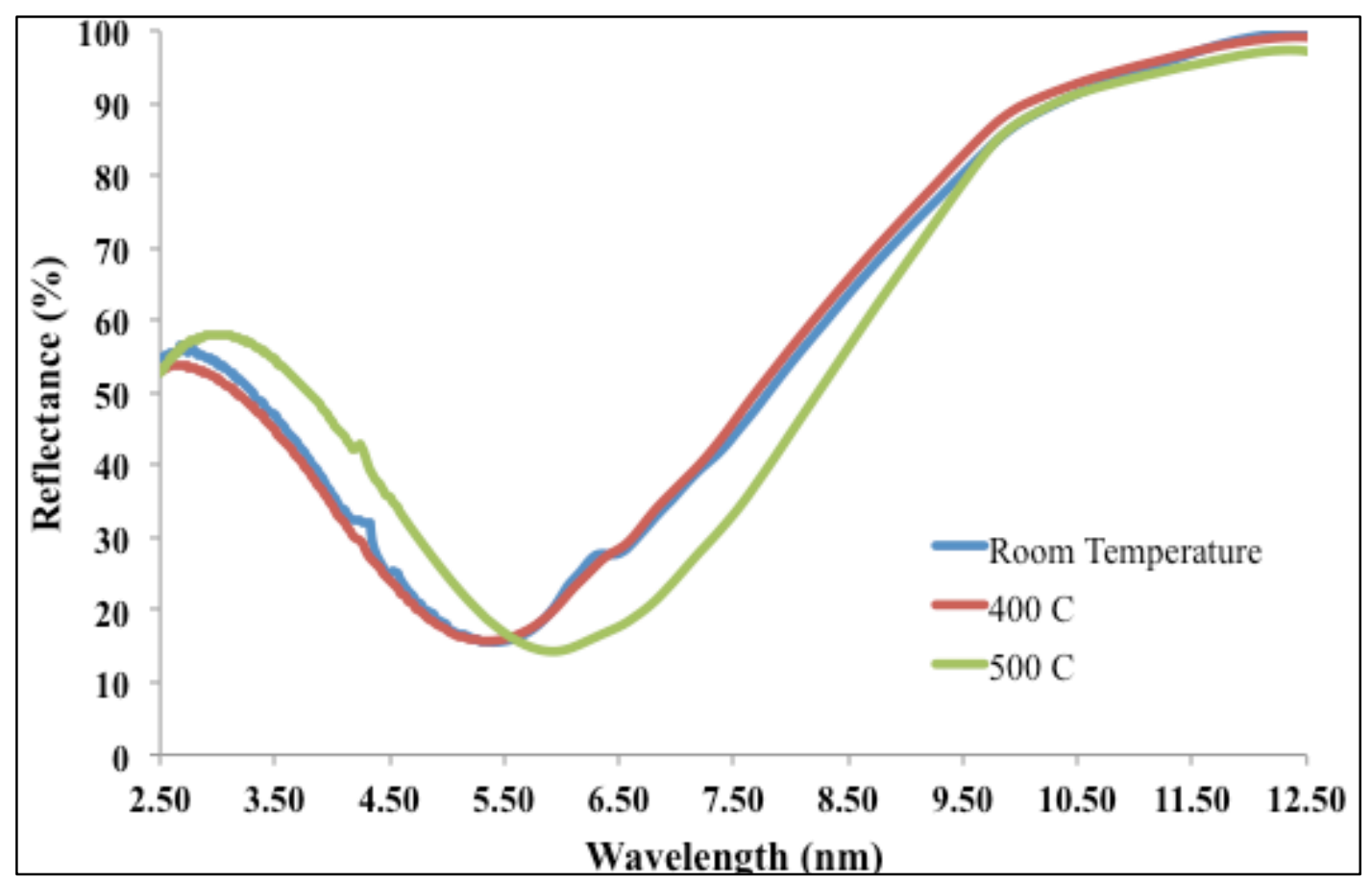

Fig 34. IR reflectance of GLAD tungsten structures before and after heat treatment. 


\section{Chapter 9. Conclusions and Future Work}

\subsection{Conclusions}

An experimental setup was developed for the fabrication of tungsten nanoparticle coating and sintering using high power laser. Laser parameters and processing conditions were optimized and sintered tungsten films were successfully fabricated on SS substrates to obtain visible light absorption and IR reflectance to achieve spectral selectivity for solar thermal applications. The surface morphology of laser sintered coatings was varied to achieve high solar absorptance and low thermal emittance. The optimized sintered coatings had surface roughness ranging from submicrons to a couple of microns. We found that two laser scans were necessary, one with low power and second with higher power, to achieve the necessary surface roughness for high solar absorptance and low thermal emittance. Sintered tungsten films with solar absorptance of $90.3 \%$ at room temperature and $\sim 87 \%$ after heat treatment at $650{ }^{\circ} \mathrm{C}$ in air for 36 hours have been achieved. The sintered coating is thermally stable at $650{ }^{\circ} \mathrm{C}$ for at least 36 hours and it maintains its performance after heat treatment. The reason for thermal stability of these coatings is due to the formation of ferberite phase that was formed while laser sintering as ferberite is stable up to $950{ }^{\circ} \mathrm{C}$ in air [46] and formation of oxide film. Achieving solar absorptance $\sim 87 \%$ and thermal emittance $\sim 49.3 \%$ has promising application as spectral selective coatings for solar tower systems. Anti-reflection $\mathrm{TiO}_{2}$ film was deposited on the laser sintered coating that further increased the solar absorptance $\sim 92.5 \%$.

Laser sintered tungsten coatings could also serve as high emittance surfaces making them ideal where thermal stability and high radiation is needed for efficient heat transfer. 
Following objectives have been accomplished:

- Deposition of tungsten nanoparticles based coating

- Development of laser sintering experimental setup

- Performed laser process parameter study and its effect on sintering of tungsten nanoparticles to find optimum laser sintering condition

- Optical and infrared properties of laser sintered coatings have been measured. Solar absorptance $\sim 90.3 \%$ before heat treatment and $\sim 87 \%$ after heat treatment at $650{ }^{\circ} \mathrm{C}$ for 36 hours

- Preliminary modeling was performed to explain IR reflection vs. wavelength behavior

- Performed optical, infrared, morphological, and elemental analysis of optimized laser sintered coating in order to understand their suitability for solar thermal applications

- XRD study indicated the presence of ferberite compound in laser sintered coating and this compound is stable at high temperatures

- Evaluated high temperature (up to $650{ }^{\circ} \mathrm{C}$ for 36 hours) behavior of laser sintered coatings. The coating was found to be stable at these conditions without any major changes in performance

- Demonstrated enhancement in performance of laser sintered $\mathrm{W}$ coating with $\mathrm{TiO}_{2}$ antireflection film of thickness $\sim 80 \mathrm{~nm}$. Solar absorptance of the coating increased to $\sim 92.5$ $\%$ barely affecting the IR spectral performance leading to having emittance $\sim 16 \%$ and $\sim 16.4 \%$ before and after applying antireflection film, respectively

\subsection{Future work}

Following tasks can be carried out for further improvements and applications: 
- Evaluation of long term stability of laser sintered coatings for thermal receivers. Laser sintered coatings need to be under heat treatment for much longer duration to study the long term effects. This is important as the operation of thermal systems is for a few years and having long term testing and performance data will be beneficial

- The laser sintering process needs to be scaled up to make it more viable for commercial use. Lasers are constantly being developed with higher powers allowing for higher energy densities on the incident material that can lead to faster sintering. A study of using high power lasers and their effect on sintering mechanism can prove helpful for making this technology more commercial ready

- Rigorous modeling needs to be carried out to correlate absorptance and emittance with surface roughness and material composition. A thorough modeling of these effects can lead to the optimum surface roughness required with necessary material composition that could achieve very high spectral selectivity. This in turn can dictate laser sintering process parameters that will result in the development of optimum surface coating 


\section{Bibliography}

[1] S.Bell, "How Do Solar Panels Work?”, truthistreason.net, http://www.truthistreason.net/howdo-solar-panels-work, 2017.

[2] C. Ungaro, Control of Optical Properties of Surfaces for Improved Solar Thermophotovoltaic Systems, University of Virginia, 2015. (Ph.D. Thesis).

[3] "Solar Thermal Power Plants", eia.gov, https://www.eia.gov/energyexplained/?page=solar thermal_power_plants, 2017.

[4] "New Parabolic Trough Collector from 3M and Gossamer", sunwindenergy.com, http://www.sunwindenergy.com/news/new-parabolic-trough-collector-3m-and-gossamer, 2017.

[5] "Power Tower Projects", nrel.gov, https://www.nrel.gov/csp/solarpaces/power_tower.cfm, 2017.

[6] C.E. Kennedy, Review of Mid- to High-Temperature Solar Selective Absorber Materials, NREL/TP-520-31267, National Renewable Energy LaboratoryGolden, CO, 2002.

[7] N. Selvakumar, H. Barshilia, Review of physical vapor deposited (PVD) spectrally selective coatings for mid- and high-temperature solar thermal applications, Solar Energy Materials and Solar Cells 98 (2012) 1-23.

[8] S. Esposito, A. Antonaia, M.L. Addonizio, and S. Aprea, Fabrication and optimisation of highly efficient cermet-based spectrally selective coatings for high operating temperature, Thin Solid Films 517 (2009) 6000-6006.

[9] A. Berghaus, A. Djahanbakhsh, and L. K. Thomas, Characterisation of CVD-tungsten alumina cermets for high-temperature selective absorbers, Solar Energy Materials and Solar Cells 54 (1998) 19-26. 
[10] P. M. Curmi, G. L. Harding, Surface texturing of copper by sputter etching with applications

for solar selective absorbing surfaces, Journal of Vacuum Science and Technology 17 (1980) $1320-1325$.

[11] A. Lasagni, M. Nejati, R. Clasen, F. Mücklich, Periodical surface structuring of metals by laser interference metallurgy as a new fabrication method of textured solar selective absorbers, Advanced Engineering Materials 8 (2006) 580-584.

[12] A. Vorobyev and C. Guo, Solar absorber surfaces treated by femtosecond laser, 2010 International Conference on Biosciences, Cancun (2010) 135-138.

[13] H. Saia, H. Yugamia, Y. Kanamorib, K. Hane, Solar selective absorbers based on two dimensional W surface gratings with submicron periods for high- temperature photothermal conversion, Solar Energy Materials and Solar Cells 79 (2003) 35-49.

[14] G. L. Harding and M. R. Lake, Sputter etched metal solar selective absorbing surfaces for high temperature thermal collectors, Solar Energy Materials 5 (1981) 445 - 464.

[15] C. Sun, Laser sintering of ultra high temperature materials, University of Virginia, Charlottesville, Virginia, 2010. (Ph.D. thesis).

[16] J. D. Majumdar and I. Manna, Laser processing of materials, Sadhana 28 (2003) 495562.

[17] Kruth, J., Levy, G., Klocke, F., Childs, T., Consolidation phenomena in laser and powder bed based layered manufacturing, CIRP Annals - Manufacturing Technology, Volume 56, Issue 2 (2007) 730-759.

[18] P. Mahalik, Micromanufacturing and Nanotechnology, Springer-Verlag Berlin Heidelberg, 2006. 
[19] S. Kunc, Rough metallic selective surfaces for solar energy applications, Solar and Wind Technolooy Vol. 3, No. 2 (1986) 147-151.

[20] A. Rahman, A. Ashraf, H. Xin, X. Tong, P. Sutter, M. D. Eisaman, and C. T. Black, Sub-50nm self-assembled nano textures for enhanced broadband antireflection in silicon solar cells. Nature Communications, 6, 5963, 2015.

[21] D. E. Aspnes and J. B. Theeten. Investigation of e_ective-medium models of micro-scopic surface roughness by spectroscopic ellipsometry. Physical Review B 8 (1979) 3292 - 3302.

[22] D. A. G. Bruggeman. Berechnung verschiedener physikalischer Konstanten von het erogenen Substanzen. I. Dielektrizittskonstanten und Leitfhigkeiten der Mischkrper aus isotropen. Annalen der Physik 416 (1935) 636- 664.

[23] G. A Nikalsson, C. G. Granqvist, and O. Hunderi. Effective medium models for the optical properties of inhomogeneous materials. Applied Optics 30 (1981) 20 - 26.

[24] H. Sai, Y. Kanamori, K. Arafune, Y. Oshita, and M. Yamaguchi. Light trapping effect of submicron surface textures in crystalline Si solar cells. Progress in Photovoltaics: Research and Applications15 (2007) 415 - 423.

[25] A. Taflove and S. C. Hagness. Computational Electrodynamics: The Finite-Difference Time-Domain Method, Third Edition. Artech House, Norwood, MA, 2005.

[26] D. M. Sullivan, Electromagnetic simulation using the FDTD method, IEEE Press, Piscataway, NJ, 2000.

[27] K. S. Yee, Numerical solution of initial boundary value problems involving Maxwell's equations in isotropic media, IEEE Transactions on Antennas and Propagation 14 (1966) 302 307.

[28] A. F. Oskooi, D. Roundy, M. Ibanescu, P. Bermel, J. D. Joannopoulos, and S. G. Johnson. 
MEEP: A flexible free-software package for electromagnetic simulations by the FDTD method. Computer Physics Communications 181 (2010) $687-702$.

[29] P. A. Davies and A. Luque, Solar thermophotovoltaics: brief review and a new look. Solar Energy Materials and Solar Cells 33 (1994) $11-22$.

[30] Y. Xuan, X. Chen, and Y. Han, Design and analysis of thermophotovoltaic systems, Renewable Energy 36 (2011) $374-387$.

[31] V. M. Andreev, V. P. Khvostikov, O. A. Khvostikova, A. S. Vlasov, P. Y. Gazaryan, N. A. Sadchikov, and V. D. Rumyantsev. Solar thermophotovoltaic system with high temperature tungsten emitter, IEEE Photovoltaic Specialists Conference (2005) $671-674$.

[32] H. Lund, E. Schaltz, D. Tonini, L.-L. P. Hansen, F. Hvelplund, J. R. Pillay, T. Astrup, H. Wenzel, B. V. Mathiesen, M. P. Nielsen, K. Heussen, L. Hamelin, P. A. Ostergaard, C. Felby, P. E. Morthorst, J. Munksgaard, P. Christensen, N. S. Bentsen, F. M. Andersen, P. Karnoe, D. Connolly, N. I. Meyer, M. Munster, and M. Lind, Coherent energy and environmental system analysis (CEESA). The Danish Council for Strategic Research Programme Commission on Sustainable Energy and Environment, 2011.

[33] A. Datas and C. Algora. Global optimization of solar thermophotovoltaic systems. Progress in Photovoltaics: Research and Applications 21 (2013) 1040 - 1055.

[34] T. DiStefano, G. Pettit, J. Woodall, J. Cuomo, Conformal anti reflective coatings on a textured tungsten surface, Appl.Phys.Lett.32 (1978) 676-678.

[35] P. Fischer, V. Romano, H.P. Weber, N.P. Karapatis, E. Boillat, R. Glardon, Sintering of commercially pure titanium powder with a Nd:YAG laser source, Acta Materialia 51 (2003) $1651-1662$.

[36] R. Glardonl, N. Karapatisl, V. Romanoz, Influence of Nd:YAG parameters on the selective 
laser sintering of metallic powders, CIRP Annals - Manufacturing Technology, Volume 50, Issue 1 (2001) 133-136.

[37] N. Parreira, T. Polcar, A. Cavaleiro, Thermal stability of reactive sputtered tungsten oxide coatings, Surf. Coat. Technol. 201 (2007) 7076-7082.

[38] J.-P. Kruth, G. Levy, F. Klocke, T. Childs, Consolidation phenomena in laser and powder bed based layered manufacturing, CIRP Ann. Manuf. Technol. 56 (2007) 730-759.

[39] S. Maier, Plasmonics: Fundamentals and applications, Springer Science + Business Media LLC, 2007.

[40] H. Sai, Y. Kanamori, K. Hane, H.Yugami, Numerical study on spectral properties of tungsten one-dimensional surface-relief gratings for spectrally selective devices J. Opt. Soc. Am. A: Opt. ImageSci. Vis. 22 (2005) 1805-1813.

[41] S. Babiker, S. Yong, M. O. Ahmed, X. Ming, and A. Abdelbagi, Design of OneDimensional Tungsten Gratings for Thermophotovoltaic Emitters, World Academy of Science, Engineering and Technology International Journal of Mathematical, Computational, Physical, Electrical and Computer Engineering 7 (2013) 1470-1474.

[42] V. Iyengar, B. Nayak, and M. Gupta, Ultralow reflectance metal surfaces by ultrafast laser texturing, Applied Optics, Vol. 49, No. 31 (2010 ) 5983-5988.

[43] C. Ungaro, S. Gray, M. Gupta, Black tungsten for solar power generation, Appl. Phys. Lett. $103(2013) 071105$.

[44] A. Taflove, S.Hagness, Computational Electrodynamics: The Finite-Difference TimeDomain Method, Artech House, Norwood,MA, 2005.

[45] A. Oskooi, D. Roundy, M. Ibanescu, P. Bermel, J. Joannopoulos, S. G. Johnson, Meep: a flexible free-software package for electromagnetic simulations by the FDTD method, 
Comput.Phys.Commun. 181 (2010) 687-702.

[46] L. C. Hsu, The stability relations of the wolframite series, Am.Mineral.61 (1976) 944-955.

[47] F. Cao, K. McEnaney, G. Chen, Z. Ren, A review of cermet-based spectrally selective solar absorbers, Energy Environ.Sci. 7 (2014) 1615-1627.

[48] A. Hall, A. Ambrosini, C. Ho, Solar selective coatings for concentrating solar power central receivers, Adv.Mater.Processes 170 (2012) 28-32.

[49] C. Ungaro, A. Shah, I. Kravchenko, D. Hensley, S. Gray, and M. Gupta, Optical and infrared properties of glancing angle-deposited nanostructured tungsten films, OPTICS

LETTERS, Vol. 40, No. 4 (2015) 506-509. 


\section{Appendix A: List of Publications}

1) C. Ungaro, A. Shah, I. Kravchenko, D. Hensley, S. Gray, and M. Gupta, Optical and infrared properties of glancing angle-deposited nanostructured tungsten films, OPTICS LETTERS, Vol. 40, No. 4 (2015) 506-509.

2) A. Shah, C. Ungaro, and M. Gupta, High temperature spectral selective coatings for solar thermal systems by laser sintering, Solar Energy Materials and Solar Cells 134 (2015) 209-214.

3) A. A. Shah and M. C. Gupta, Spectral selective surfaces for concentrated solar power receivers by laser sintering of tungsten micro and nano particles, Solar Energy Materials and Solar Cells 117 (2013) 489-493.

4) A. Shah and M. Gupta, Laser texturing for solar thermal systems, Conference on Lasers and Electro-Optics, May 2011. 


\section{Appendix B: Published Work}




\title{
Optical and infrared properties of glancing angle-deposited nanostructured tungsten films
}

\author{
Craig Ungaro, ${ }^{1}$ Ankit Shah, ${ }^{1}$ Ivan Kravchenko, ${ }^{2}$ Dale K. Hensley, ${ }^{2}$ Stephen K. Gray, ${ }^{3}$ and Mool C. Gupta ${ }^{1, *}$ \\ ${ }^{1}$ Department of Electrical \& Computer Engineering, University of Virginia, Charlottesville, Virginia 22901, USA \\ ${ }^{2}$ Center for Nanophase Materials Sciences, Oak Ridge National Laboratory, Oak Ridge, Tennessee 37831, USA \\ ${ }^{3}$ Center for Nanoscale Materials, Argonne National Laboratory, 9700 South Cass Avenue, Argonne, Illinois 60439, USA \\ ${ }^{*}$ Corresponding author: mgupta@virginia.edu
}

Received October 31, 2014; accepted December 17, 2014;

posted January 7, 2015 (Doc. ID 226082); published February 6, 2015

\begin{abstract}
Nanotextured tungsten thin films were obtained on a stainless steel (SS) substrate using the glancing-angledeposition (GLAD) method. It was found that the optical absorption and thermal emittance of the SS substrate can be controlled by varying the parameters used during deposition. Finite-difference time-domain (FDTD) simulations were used to predict the optical absorption and infrared (IR) reflectance spectra of the fabricated samples, and good agreement was found between simulated and measured data. FDTD simulations were also used to predict the effect of changes in the height and periodicity of the nanotextures. These simulations show that good control over the absorption can be achieved by altering the height and periodicity of the nanostructure. These nanostructures were shown to be temperature stable up to $500^{\circ} \mathrm{C}$ with the addition of a protective $\mathrm{HfO}_{2}$ layer. Applications for this structure are explored, including a promising application for solar thermal energy systems. @ 2015 Optical Society of America

OCIS codes: (160.4236) Nanomaterials; (160.4670) Optical materials; (160.4760) Optical properties; (230.4000) Microstructure fabrication; (240.6700) Surfaces; (350.6050) Solar energy.

http://dx.doi.org/10.1364/OL.40.000506
\end{abstract}

Glancing-angle deposition (GLAD) is a technique that allows a nanostructured thin film to be deposited on a substrate via vapor deposition or sputtering. The features of this deposited film can be controlled by varying the substrate spin speed, angle of deposition, and deposition rate and time [1]. GLAD has been used to form tungsten nanostructures $[\underline{2}, \underline{3}]$ for different applications. Here, we report the control of optical and infrared (IR) properties of nanostructured tungsten (W) thin films using the GLAD method. A hafnium oxide $\left(\mathrm{HfO}_{2}\right)$ layer was deposited on top of the nanostructures to act as an antireflection coating and provide protection against oxidation [4]. We also present data on optical and IR properties simulated using the finite-difference timedomain (FDTD) method. Simulations and experiments show that the optical absorbance and IR emittance of the surfaces can be controlled by altering the features of the nanostructures. These surfaces can be used for a variety of applications, including control of hydrophobicity, sensing, IR sources, and solar energy harvesting.

One of the applications of spectrally selective surfaces is in concentrated solar power (CSP) using solar thermal tower systems. Solar thermal tower systems are capable of providing $>300 \mathrm{MW}$ of power and are highly important for utility scale power generation [5]. Mirrors on the ground concentrate sunlight onto a central receiver, and concentrations of 600 -fold can be achieved [6]. As a result of this high concentration, receivers in the tower systems reach extremely high temperatures, with temperatures $>600^{\circ} \mathrm{C}$ desired to meet an electricity cost target of $0.06 / \mathrm{kWh}$ [7]. Receivers for tower systems require solar absorbance $(\alpha)>0.95$ and thermal emittance $(\epsilon)$ $<0.3$ and must be able to operate in air at high temperatures [6]. To achieve these requirements, receivers are coated with a material that has low reflectance in the solar spectrum and high reflectance in the IR; this behavior is called spectral selectivity [].

Multilayers of ceramic-metal composites (cermets) have been used to form spectral selective coatings, but their performance degrades due to oxidation, cracking, and delamination at high temperatures [9]. Cermets have only been stable up to $500^{\circ} \mathrm{C}$ in air [10] and, as mentioned earlier, there is a need to go to higher temperatures. Surface texturing has also been used to obtain spectral selectivity that can achieve relatively high solar absorbance, but maintaining low emittance is a challenge $[11,12]$. Sub-micron periodic tungsten structures have been fabricated on a tungsten substrate that achieved $\alpha$ of $82 \%$ and $\epsilon$ of $15.9 \%$ at $1200 \mathrm{~K}$; however, the heat treatment was carried out only in vacuum [12]. A chromium surface was textured to achieve an $\alpha$ of $94 \%$ and an $\epsilon$ of $30 \%$ at $100^{\circ} \mathrm{C}$ after being oxidized in air at $500^{\circ} \mathrm{C}$, and it can be seen that surface textures are either stable under vacuum at high temperatures or in air up to $500^{\circ} \mathrm{C}[\underline{13}]$.

GLAD structures offer a way to achieve the high level of control over the optical properties of a surface that is needed for solar applications without the need for periodic nanostructures. GLAD using sputtering can be applied to a wide area without the need for lithography techniques, and the aperiodicity of the GLAD structures also makes them more tolerant of small changes in surface morphology due to high temperature operation.

Spectrally selective surfaces can also be used as IR sources since they are needed for IR-based analytic systems [14]. Specifically, carbon dioxide gas sensor systems employ IR sources and detectors. Most IR sources are very broadband; therefore, narrow band emission is needed that corresponds to particular gas concentration and detection. In the paper by Lai $e t$ al., the design of a MEMS-based IR emitter and detector as a gas sensor 
system was considered, and the IR emission was centered at a wavelength of $4.25 \mu \mathrm{m}$ [15].

In this work, we show that fabricated GLAD tungsten nanostructures have an emission at $5 \mu \mathrm{m}$ and are thermally stable. The IR emission center wavelength can be controlled by changing the height of the structures, thus GLAD structures would be very useful as IR emitters in gas sensor systems. Another application of spectral selective surfaces is in filters. The surface can be used as a mirror that reflects IR radiation and absorbs visible radiation with a cutoff that is tunable via the GLAD deposition parameters.

GLAD of tungsten nanostructures was carried out on a stainless steel (SS) substrate. The deposition angle used was $85^{\circ}$ from the normal of the surface, and the sample rotation speed was $20 \mathrm{rpm}$. The sample was kept at room temperature. The electron beam evaporator chamber vacuum was $4 \times 10^{-3} \mathrm{~Pa}$.

$\mathrm{HfO}_{2}$ layers were grown via atomic layer deposition at a substrate temperature of $563 \mathrm{~K}$, with the full deposition process as described by Balke et al. [16]. Significant oxidation of the substrate is unlikely at a deposition temperature of $563 \mathrm{~K}$; however, a native oxide of unknown thickness will be present on the tungsten substrate at room temperature. Solar absorbance measurements were performed using an integrating sphere supplied by Labsphere Inc. and using individual lasers having wavelengths 405, 532, 633, 790, 980, and $1064 \mathrm{~nm}$.

IR reflectance measurements were performed using a continuum microscope that was purchased from Thermo Fisher Scientific, Inc. The sample was illuminated with IR radiation, and the reflected beam was collected with a $\pm 35^{\circ}$ acceptance angle from the normal. In order to study high temperature performance of the nanostructures, the sample was heated to $400^{\circ} \mathrm{C}$ and then to $500^{\circ} \mathrm{C}$ for $1 \mathrm{~h}$ in air. Emittance at high temperatures was calculated from experimental reflectance data measured for heat-treated samples using blackbody irradiance at $500^{\circ} \mathrm{C}$.

Energy dispersive x-ray spectroscopy (EDS) measurements were carried out for GLAD tungsten nanostructures without a $\mathrm{HfO}_{2}$ coating. The samples were tilted toward the detector during the EDS measurement to avoid x-ray attenuation by the sample itself. The resulting angle between the samples and the detector was $5^{\circ}$. The spot size of the electron beam in the EDS measurement was $1 \mathrm{~nm}$ in diameter; however, $\mathrm{x}$ rays were excited and collected from a much larger area. The EDS data is as follows: $25 \%$ Tungsten, $49 \%$ Iron, $13 \%$ Chromium, $5 \%$ Nickel, 1\% Manganese, 4\% Carbon, and 3\% Oxygen. Iron, chromium, nickel, and manganese are in EDS data due to SS composition.

Simulations of these nanostructures were performed with the FDTD method using the MIT electromagnetic equation propagation (MEEP) code $[17,18]$. The FDTD method finds a solution to a system by iteratively solving Maxwell's equations over a grid. A Gaussian source was used to excite electric and magnetic fields, and structures were specified via the dielectric constants of the grid at each location. Perfectly matched layer (PML) boundary conditions were used in the direction of light propagation, while Bloch periodic boundary conditions were used in the lateral directions. Discretization errors were avoided by using a small (1-nm) resolution and

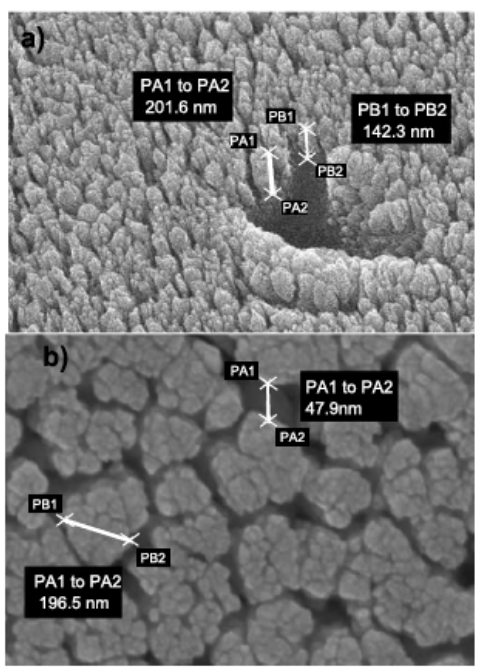

Fig. 1. SEM image of tungsten nanostructures on SS substrate (a) tilted view and (b) top view.

sub-pixel averaging [19]. To calculate the reflected power, a simulation is first run with no object, to obtain the incident wave power, then another simulation is run with the object present. The electromagnetic flux from the first simulation is then subtracted from the second to obtain the reflected wave. Unpolarized light was approximated by averaging the reflectance spectra for $\mathrm{S}$ and $\mathrm{P}$ polarized light. The dimensions of the structure were determined by the scanning electron microscope (SEM) images shown in Fig. $\underline{1}$.

A Drude-Lorentz model was used to simulate dielectric constants in the wavelength range. In this model, intra-band, or free-electron effects, are described by a Drude term, while inter-band, or bound-electron effects, are described by the addition of multiple Lorentzian terms. Specific constants for tungsten are given by Rakic et al. [20].

Figure 1 shows the SEM image of tungsten nanostructures on SS substrate. The nanostructures are randomly arranged, and the height of the nanostructures varies from 130 to $202 \mathrm{~nm}$ as seen in Fig. 1(a). As seen in Fig. 1(b), the diameter of the nanostructures varies from 40 to $100 \mathrm{~nm}$, and the spacing between them varies from 10 to $50 \mathrm{~nm}$. Small-scale surface roughness is seen on the tops of the nanostructures.

Experimental studies of GLAD structures of similar dimensions show a RMS roughness of 5.8 [21] and $4.3 \mathrm{~nm}$ [22]. It was found that surface roughness of RMS $<5.8 \mathrm{~nm}$ had little effect on the absorbance of the surfaces at the wavelengths investigated in this report. Nanoscale crystallinity of the tungsten nanostructure is not taken into account in this report. While it is an important factor in calculating the absorbance spectra of many nanostructures, it is unlikely to have a large effect on this research due to the short electron mean-free path $(19.1 \mathrm{~nm})$ combined with the typically large grain size in GLAD deposited tungsten, and the lack of significant edge effects due to the size of the structures considered $[3,23,24]$. The randomness of nanostructures helps to achieve high solar 


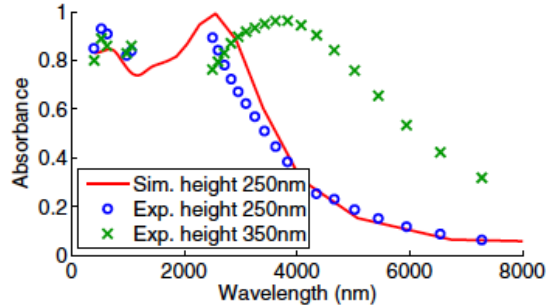

Fig. 2. Comparison between experiment and FDTD modeling of absorption spectra.

absorbance and spectral selectivity important for solar thermal applications. This occurs due to a widening of the absorption peak as the radii of the nanostructures become more random $[25,26]$.

Figure 2 shows a comparison of the absorption spectra for simulated and experimental results. The nanostructure was simulated by tracing the nanostructure edges in a top view SEM image to create a $2 \mathrm{D}$ model of the surface in a $5 \times 5 \mu \mathrm{m}$ area using a common imaging tracing technique [27]. The 2D image was then extended into 3 dimensions by adding the height and small $\sim 5$-nm surface roughness obtained from an angled SEM image. This information was used to define the tungsten structure used by the FDTD simulations. Internal reflection between the tungsten layer and the SS substrate is taken into account in this simulation. It can be seen from this data that there is a good correlation between simulated and experimental results at different heights for the tungsten nanostructure. Discrepancies between experimental and simulated data are due to SEM resolution limits and the size of the area simulated.

In Fig. 3, similar nanostructures of tungsten are simulated with various heights. It can be seen from the graph that increasing the nanostructure's height shifts the absorbance peak to longer wavelengths, and decreases the height of the peak. In Fig. 4, it can be seen that the height of the absorbance peak can be controlled via the average distance between the centers of adjacent structures. In this way, we can control the position and height of the absorbance peak in GLAD structures. This control allows tuning of the spectrally selective surface to be used for a variety of applications.

The $\mathrm{W}$ nanostructures discussed in this report are subwavelength compared to the wavelengths in the solar and IR spectrum as seen earlier in the SEM images. Increased absorption occurs due to Fabry-Perot resonances occurring in the nanostructured area formed by GLAD.

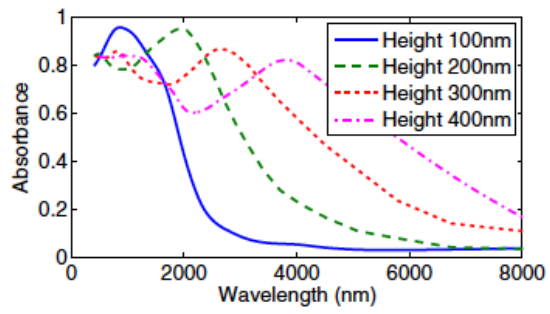

Fig. 3. FDTD simulation results for absorbance by tungsten nanostructures with various heights.

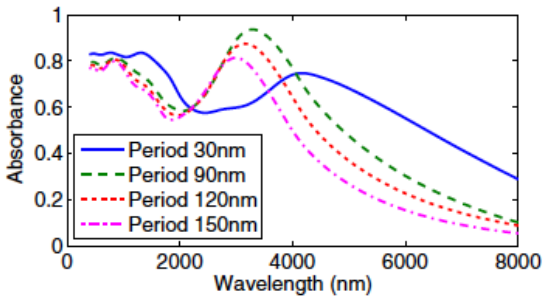

Fig. 4. FDTD simulation results for absorbance by tungsten nanostructures with various periods. Note that periodicity here refers to the average distance between nanostructure centers.

In addition to this, the $\mathrm{HfO}_{2}$ layer acts as an antireflection coating, thus reducing front surface reflection. The combined effect of the Fabry-Perot type behavior and the anti-reflection from the coating leads to a very high solar absorbance of $87.4 \%$.

Figure $\underline{5}$ shows an IR absorbance plot of the W nanostructures with $\mathrm{HfO}_{2}$ coating before and after heat treatment. The peak in absorbance between 4 and $6 \mu \mathrm{m}$ occurs due to the Fabry-Perot resonances that occur in the GLAD structure. Maxwell-Garnett effective medium theory can be used to predict the locations of the resonances and their shift with height [28]. Beyond wavelength of $5.5 \mu \mathrm{m}$, the absorbance starts falling and approaching that of bulk tungsten as the structure dimensions are much smaller than the wavelength.

A slight shift to the right can be seen in the IR absorbance after heating the sample at $500^{\circ} \mathrm{C}$ in air for $1 \mathrm{~h}$; this could be due to a change in the large-scale surface morphology of the GLAD film due to diffusion or recrystallization at high temperatures $[\underline{4}, \underline{29}]$. Flat tungsten substrates have been shown to grow 3 . $5 \mathrm{~nm}$ of oxide under similar experimental conditions [30]. However, the structures still maintain their performance, as beyond $6.5 \mu \mathrm{m}$ the absorbance approaches that of bulk tungsten and also the solar absorbance remains unchanged.

This Letter demonstrates the use of the GLAD method to achieve a surface with high optical absorption and low thermal emittance. Solar absorbance and thermal emittance data are presented as well. Thermal stability of the structure is shown up to $500^{\circ} \mathrm{C}$ in air with a protective $\mathrm{HfO}_{2}$ layer. Electrodynamic simulation results also show a path forward toward increased efficiency by using shorter tungsten nanostructures. This research is very promising for solar thermal applications, and to the authors' knowledge, is the first demonstration of the GLAD method for these systems.

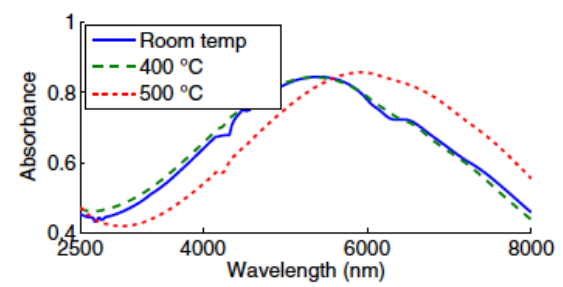

Fig. 5. Change in absorbance after heat treatment. 
We thank the NASA Langley Professor and NSF IUCRC Programs for their support of this project. This work was performed, in part, at the Center for Nanoscale Materials, a U.S. Department of Energy, Office of Science, Office of Basic Energy Sciences User Facility under Contract No. DE-AC02-06CH1135. A portion of this research was conducted at the Center for Nanophase Materials Sciences, which is sponsored at Oak Ridge National Laboratory by the Scientific User Facilities Division, Office of Basic Energy Sciences, U.S. Department of Energy.

\section{References}

1. J. C. Sit, D. Vick, K. Robbie, and M. J. Brett, J. Mater. Res 14, 1197 (1999).

2. K. R. Khedir, G. K. Kannarpady, H. Ishihara, J. Woo, C. Ryerson, and A. S. Biris, Phys. Lett. A 374, 4430 (2010).

3. D. Deniz, D. J. Frankel, and R. J. Lad, Thin Solid Films 518 , 4095 (2010).

4. L. A. Arpin, M. D. Losego, and P. V. Braun, Chem. Mater. 23 4783 (2011).

5. NREL: Concentrating Solar Power Ivanpah solar electric generating systemhttp://www.nrel.gov/csp/solarpaces/ project_detail.cfm/projectID=62, accessed: $2014-07-31$.

6. A. Ambrosini, T. N. Lambert, M. Bencomo, A. Hall, K. van Every, N. P. Siegel, and C. K. Ho, Proc. ASME 5, (2011).

7. A. Hall, A. Ambrosini, and C. Ho, Adv. Mater. Processes 170, 28 (2012)

8. S. Fan, Nat. Nanotechnol. 9, 92 (2014).

9. R. N. Schmidt and K. C. Park, Appl. Opt. 4, 917 (1965).

10. F. Cao, K. McEnaney, G. Chen, and Z. Ren, Energ. Environ. Sci. 7, 1615 (2014).

11. A. Y. Vorobyev and G. Chunlei, Proc. IEEE 7, 135 (2010).

12. H. Sai, H. Yugamia, Y. Kanamorib, and K. Hane, Sol. Energy Mater. Sol. Cells 79, 35 (2003).

13. S. Kunc, Sol. Wind Technol. 4, 473 (1987).
14. J. Hodgkinson and R. P. Tatam, Meas. Sci. Technol. 24, 012004 (2013).

15. J. J. Lai, H. F. Liang, Z. L. Peng, X. Yi, and X. F. Zhai, J. Phys. Conf. Ser. 276, 012129 (2011)

16. N. Balke, P. Maksymovych, S. Jesse, I I. Kravchenko, Q. Li, and S. V. Kalinin, ACS Nano 8, 10229 (2014).

17. A. Taflove and S. C. Hagness, Computational Electrodynamics: The Finite-Difference Time-Domain Method, 3rd ed. (Artech House, 2005).

18. A. F. Oskooi, D. Roundy, M. Ibanescu, P. Bermel, J. D. Joannopoulos, and S. G. Johnson, Comput. Phys. Commun. 181, 687 (2010)

19. A. Deinega and I. Valuev, Comput. Phys. Commun. 182, 149 (2011).

20. A. D. Rakic, A. B. Djurisic, J. M. Elazar, and M. L. Majewski, Appl. Opt. 37, 5271 (1998).

21. S. Wang, X. Fu, G. Xia, J. Wang, J. Shao, and Z. Fan, Appl. Surf. Sci. 252, 8734 (2006).

22. J. Lintymer, N. Martin, J.-M. Chappe, P. Delobelle, and J. Takadoum, Surf. Coat. Technol. 180-181, 26 (2004).

23. R. Atkinson, W. R. Hendren, G. A. Wurtz, W. Dickson, A. V. Zayats, P. Evans, and R. J. Pollard, Phys. Rev. B 73, 235402 (2006).

24. D. Choi, C. S. Kim, D. Naveh, S. Chung, A. P. Warren, N. T. Nuhfer, M. F. Toney, K. R. Coffey, and K. Barmak, Phys. Rev. B 86, 045432 (2012).

25. T. Jun, L. Yong-Hua, Z. Rong-Sheng, L. Kai-Qun, X. Zhi-Guo, L Zhao-Feng, L Sheng-li, W. Pei, and M. Hai, Chin. Phys. Lett. 25, 4459 (2008).

26. C. Ungaro, S. K. Gray, and M. C. Gupta, Appl. Phys. Lett. 103, 071105 (2013)

27. J. C. Fiala, J. Microsc. 218, 52 (2005).

28. L Hu and G. Chen, Nano Lett. 7, 3249 (2007).

29. C. Schlemmer, J. Aschaber, V. Boerner, and J. Luther, AIP Conf. Proc. 653, 164 (2003).

30. J. Habainy and C. Nilsson, "Oxidation of pure tungsten in the temperature interval $400^{\circ}$ to $900^{\circ} \mathrm{C}$," Master's thesis (Lund University, 2013). 


\title{
High temperature spectral selective coatings for solar thermal systems by laser sintering
}

\author{
Ankit A. Shah, Craig Ungaro, Mool C. Gupta* \\ Department of Electrical and Computer Engineering, University of Virginia, Charlottesville, VA 22904, USA
}

A R T I C L E I N F O

\section{Article history:}

Received 23 May 2014

Received in revised form

24 October 2014

Accepted 3 November 2014

Available online 16 December 2014

Keywords:

Spectral selective coating

Central tower systems

Concentrating solar thermal

Laser sintering

Tungsten

Nano particles

\begin{abstract}
A B S T R A C T
Spectral selective coatings for solar thermal receivers have been fabricated by laser sintering of tungsten micro and nano particles on stainless steel substrate and show solar absorptance of $\sim 90 \%$ at room temperature. The coatings remain stable after heat treatment at $650{ }^{\circ} \mathrm{C}$ in air for at least $36 \mathrm{~h}$. A high figure of merit of $\sim 50$ has been achieved for laser sintered coatings. Two laser scans were performed at different energy densities, one to sinter nanoparticles and other to control surface morphology, so that high solar absorptance and low thermal emittance can be achieved. Laser sintering was carried out under atmospheric pressure that enabled the formation of a protective oxide layer that provided thermal stability to $650{ }^{\circ} \mathrm{C}$. Solar thermal receivers in tower systems operate at high temperatures in air and laser sintering is a promising approach to fabricate spectral selective receiver coatings for this application.
\end{abstract}

c) 2014 Elsevier B.V. All rights reserved.

\section{Introduction}

One of the major goals of concentrating solar power (CSP) systems is to efficiently convert sunlight into thermal energy, which is used to generate electricity. CSP systems use mirrors to concentrate sunlight onto a receiver that transfers heat to the internal circulating fluid called heat transfer fluid (HTF). The heat from HTF is then utilized by the power cycle to convert from thermal to electric power. CSP systems are of various types that include parabolic trough, solar dish, and central tower. One of the most promising is the central tower system. Mirrors on the ground focus sunlight onto a receiver that sits on top of the tower. For a higher overall electrical efficiency, receivers need to have high photothermal conversion efficiency at very high operating temperatures. This requires solar tower receivers to efficiently absorb most of the solar light and retain the heat energy to be carried effectively by HTF. This characteristic behavior of the receivers corresponds to having high solar light absorptance $(\alpha)$ and low thermal emittance $(\varepsilon)$ in the infrared (IR). Thus receivers have to be coated with spectrally selective coatings that have $\alpha>0.95$ at wavelengths $(\lambda)<2.5 \mu \mathrm{m}$ and $\varepsilon<0.3$ [1], thus requires high reflectance in the infrared at $\lambda>2.5 \mu \mathrm{m}$. In order for CSP systems to meet an electricity cost target of $\$ 0.06 / \mathrm{kW} \mathrm{h}$, new materials that are capable to perform at temperatures $>600{ }^{\circ} \mathrm{C}$ are needed [2].

\footnotetext{
* Corresponding author. Tel.: + 17573256850 .

E-mail address: mgupta@virginia.edu (M.C. Gupta).
}

Spectral selective coatings have different requirements based on the specific CSP system. For example, receivers in parabolic trough systems operate with sunlight concentration $\sim 70-80$ at temperatures $\sim 450{ }^{\circ} \mathrm{C}$ in vacuum and require $\alpha>0.90$ and $\varepsilon<0.1$. However, when the vacuum is breached in trough tubes the coatings can degrade due to oxidation [3]. Tower systems usually have a much higher sunlight concentration $\sim 600$ [1] and require $\alpha>0.95$ and $\varepsilon<0.3$ [1]. Multilayers of ceramic-metallic composites (cermets) have been used as solar selective coatings [4-10], but their performance degrades in air due to oxidation at high temperatures, diffusion of metal particles, delamination of coatings, and cracking. To the best of our knowledge, cermet coatings have been stable only up to $500{ }^{\circ} \mathrm{C}$ in air [11] and there is a need to develop coatings that operate at higher temperatures. Currently, Pyromark 2500 series paint is the standard for central tower receivers that has $\alpha \sim 0.96$ and $\varepsilon \sim 0.86$ and therefore has high thermal losses [2]. This paint also degrades over time that leads to lower performance and added cost to CSP systems [2].

In order to evaluate performance of spectral selective coatings, the use of a figure of merit (FOM) is suggested in [1] for solar tower receiver application. FOM is given as follows:

FOM $\left(\mathrm{W} / \mathrm{cm}^{2}\right)=60 \alpha_{\text {solar }}-5\left[\frac{\left(\varepsilon_{80 \mathrm{c}}+\varepsilon_{2400 \mathrm{~nm}}\right)}{2}\right]$

where $\alpha_{\text {solar, }} \varepsilon_{80 \mathrm{c}}$, and $\varepsilon_{2400 \mathrm{~nm}}$ are solar absorptance, total emittance at $80^{\circ} \mathrm{C}$, and at $2400 \mathrm{~nm}$ wavelength, respectively. $\varepsilon_{2400 \mathrm{~nm}}$ is assumed to be equal to the absorptance at $2400 \mathrm{~nm}$ according to Kirchoffs Law. The constants 60 and 5 are used to correspond to the energy flux incident on the receiver and the 
energy flux emitted at $700{ }^{\circ} \mathrm{C}$ into an environment of $20^{\circ} \mathrm{C}$. The emittance term basically provides an average value over the wavelength spectrum of interest for the energy flux emitted [1]. In the study carried out in [1], metal oxide spinels were deposited as solar selective coatings on stainless steel (SS) coupons due to their high temperature oxidation stability. Dip coating, spin coating, and thermal spray techniques were used to deposit metal oxide spinel coatings and their FOM was calculated. A FOM in the range of 46.0-51.5 was achieved for spinel oxide coatings that were fabricated using spin coating technique, however, it took several hours for fabrication [1]. A FOM in the range of 35.0-48.0 and $40-52$ was obtained for coatings prepared by thermal spray technique before and after heat treatment, respectively, but they have yet to optimize this method for CSP receiver applications [1].

In this paper, laser sintering of tungsten (W) nanoparticles on a stainless steel (SS) substrate was conducted to form spectral selective coatings. Laser sintering can take place in form of solid state sintering or liquid phase sintering. During solid state sintering, the consolidation of powder takes place at temperature much lower than the melting point and the binding occurs due to diffusion of atoms among adjacent particles [12]. During liquid phase sintering, partial melting can take place that involves raising the temperature of the particles such that only the surface of the particles melt and the core remains in solid state. The liquid formed due to the melted surface flows between the particles joining them to provide a consolidated layer $[12,13]$. Not only partial melting, but also full melting of the particles can take place during liquid phase sintering and dense layers can be formed due to this mechanism as well. As seen later, the sintered layer that is presented in this paper is formed mainly due to full melting of the particles.

We demonstrate higher level of performance of our laser processed coatings [14] and also report results on high temperature stability. In the second laser-scanning step, we did not drop cast tungsten powder again; instead we increased the laser energy density and rescanned the first layer. This two-step scanning process allows sintering of nanoparticles and control of surface morphology. This process helped to achieve the desired surface roughness which in turn increased $\alpha$ from $\sim 83 \%$ in [14] to $\sim 90 \%$. This increase in solar absorptance is critical because with data presented in [14] the FOM would have been $\sim 46$ as compared to $\sim 50$ that is achieved with coating described in this paper. A FOM of $\sim 50$ is comparable to the Pyromark 2500 series paint but Pyromark 2500 only maintains integrity up to $527^{\circ} \mathrm{C}$ and lacks significant manufacturing repeatability as discussed by authors in [15]. Laser sintered coating data presented in this paper show that it maintains its performance fairly well after heat treatment in air at $650{ }^{\circ} \mathrm{C}$ for at least $36 \mathrm{~h}$.

The laser sintered coating would be applicable for central tower systems because it can meet the desired operating temperature of $>600{ }^{\circ} \mathrm{C}$ and can be operated in air. As mentioned earlier, this high temperature operation will enable to meet the goal of $\$ 0.06 / \mathrm{kW} \mathrm{h}$. In central tower systems, maximization of solar absorptance is highly important since the magnitude of the incoming energy flux is extremely high ( $\sim 600$ sun) as it operates under high concentration. Also, controlling the emittance benefits, if not as much as absorptance, the overall selective absorber efficiency as demonstrated later in the paper. This is not the case with parabolic trough systems where the incoming energy flux is $\sim 10$ times less and hence the coatings are required to have a very low emittance.

\section{Experiment}

Pulsed laser sintering of tungsten particles on stainless steel (SS) substrates is performed to form solar selective coatings. W micro
$(1-5 \mu \mathrm{m})$ and nano $(80-100 \mathrm{~nm})$ particles were purchased from SkySpring Nanomaterials Inc. and the experimental procedure for laser sintering is very similar to the one described in [14]. Fig. 1 shows the laser sintering concept.

Laser wavelength of $1064 \mathrm{~nm}$, pulse frequency of $50 \mathrm{kHz}$, and a scan speed for the X-Y stage of $1 \mathrm{~mm} / \mathrm{s}$ was selected for sintering. The laser energy density used during first scan for sintering $\mathrm{W}$ particles was $0.8-1.3 \mathrm{~J} / \mathrm{cm}^{2}$. Below energy density of $0.8 \mathrm{~J} / \mathrm{cm}^{2}$, no melting of the tungsten particles to create a bond with SS; therefore a very discontinuous coating was formed with only partial bonding taking place with the substrate. Energy density between 0.8 and $1.3 \mathrm{~J} / \mathrm{cm}^{2}$ was sufficient to achieve a thin continuous coating of sintered tungsten on SS. Between 1.3 and $1.6 \mathrm{~J} / \mathrm{cm}^{2}$ a yellowish-white layer on sintered region was formed due to formation of tungsten trioxide as confirmed by XRD. Between 1.6 and $2.6 \mathrm{~J} / \mathrm{cm}^{2}$ a continuous layer with different surface morphology is observed due to different sintering kinetics and surface tension effects of the melt pool.

After the first laser scan, this sintered region was laser scanned for the second time without tungsten drop casting with energy density of $1.6-2.6 \mathrm{~J} / \mathrm{cm}^{2}$. The first laser scan was carried out to obtain a continuous layer of tungsten on SS substrate without any voids. The second laser scan was used to modify the surface roughness of already sintered tungsten layer to obtain desired surface morphology that resulted in higher solar absorptance and lower emittance values. Due to difference in energy coupling between powder and sintered layer, we see a variation in surface morphology. Energy density higher than $2.6 \mathrm{~J} / \mathrm{cm}^{2}$ achieved a greater surface roughness that resulted in lower reflectance in the IR causing higher emittance values. Surface roughness increases with increasing laser power [12]. More liquid formation and vaporization takes place and after solidification a greater surface roughness is observed [13]. Therefore, choosing the energy density between 1.6 and $2.6 \mathrm{~J} / \mathrm{cm}^{2}$ for our second scan provided the necessary optimized roughness for good spectral selectivity. Solar absorptance measurements were done using an integrating sphere, purchased from Labsphere Inc., and laser wavelengths of $410 \mathrm{~nm}, 532 \mathrm{~nm}$, $633 \mathrm{~nm}, 790 \mathrm{~nm}, 980 \mathrm{~nm}$, and $1064 \mathrm{~nm}$. Reflectance measurements in the IR were done using a continuum IR microscope that was purchased from Thermo Fisher Scientific Inc. The sample was illuminated by IR wavelengths ranging from 2.5 to $12.5 \mu \mathrm{m}$ and then reflected radiation power was measured to provide a reflectance versus wavelength curve. The IR microscope has the ability to collect reflected and scattered light at an angular range of $\pm 35^{\circ}$ from normal to the sample. In order to study the high temperature performance of the sintered coatings, separate samples were heated to $300^{\circ} \mathrm{C}, 400{ }^{\circ} \mathrm{C}, 500^{\circ} \mathrm{C}$, and $600^{\circ} \mathrm{C}$. No significant change was observed in $\alpha$ and $\varepsilon$ values. In this paper, we present the data for sintered coating that was heated in air for $2 \mathrm{~h}, 12 \mathrm{~h}$, and then $36 \mathrm{~h}$ at $650^{\circ} \mathrm{C}$ since this is a more desirable temperature operation range for higher efficiencies in tower systems. Following the heat treatment, both solar absorptance and IR reflectance measurements were carried out and emittance at temperature of $80^{\circ} \mathrm{C}$ was calculated based on room temperature experimental reflectance

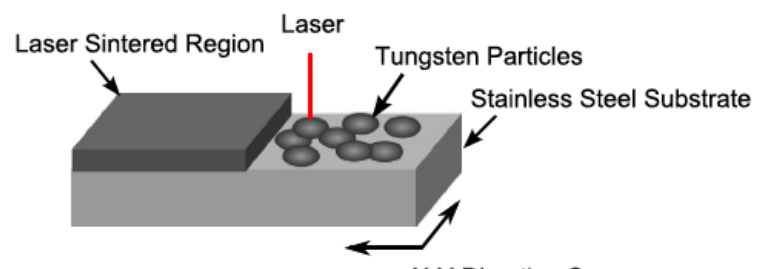

$\mathrm{X}-\mathrm{Y}$ Direction Scan

Fig. 1. Laser is scanned across the sample melting the metallic particles causing them to bind together to form a sintered layer. 
data and $80^{\circ} \mathrm{C}$ blackbody irradiance spectrum. X-ray diffraction measurements were carried out on sintered coating before and after heat treatment using X-ray diffraction system purchased from Rigaku Corporation involving a $\mathrm{CuK} \alpha$ radiation source. Energy dispersive X-ray spectroscopy (EDS) measurements were carried out on laser sintered sample before and after heat treatment to evaluate oxidation behavior.

\section{Results and discussion}

\subsection{Morphological and crystal structure study of laser sintered tungsten}

SEM images of the laser sintered tungsten sample are shown in Fig. 2a-d. We can see from the SEM images that sintered layers are continuous and without voids. We observe peaks and valleys arranged randomly which were formed due to the second laser scan
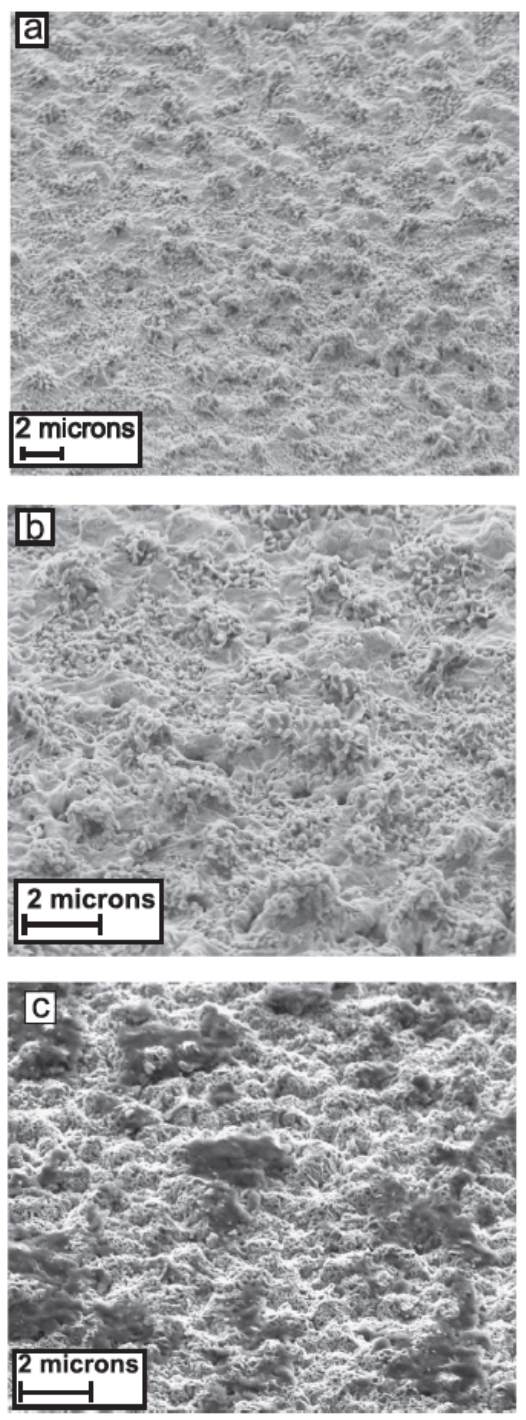

Fig. 2. SEM images of laser sintered tungsten (a) lower magnification, (b) higher magnification, and (c) higher magnification after heat treatment at $650^{\circ} \mathrm{C}$ for $2 \mathrm{~h}$. performed to modify surface roughness. The roughness has random spacing from microns to submicron scale. Fig. 3a shows XRD spectra of the laser sintered coating and there is no change before and after heat treatment at $650^{\circ} \mathrm{C}$ after $2 \mathrm{~h}$. We observe ferberite $\left(\mathrm{FeO}_{4} \mathrm{~W}\right)$ peaks along with iron peaks from stainless steel. The coating is $\sim 1 \mu \mathrm{m}$ thick so the X-rays can penetrate the coating and pick up the stainless steel (iron) signal. The ferberite phase is formed during the laser sintering process because very high temperatures are reached and so the tungsten and iron in presence of air form this compound. It has been reported that ferberite is stable upto $950{ }^{\circ} \mathrm{C}$ in air [16] and that explains the thermal stability of the laser sintered coating. This is the first observation of ferberite formation by laser sintering method. The peak positions were identified based on the reported X-ray diffraction data (Card no. 9000224 in Rigaku Corp. software database). We did not observe tungsten elemental peaks. Fig. 3b shows XRD spectra of sintered coating after heat treatment at $650^{\circ} \mathrm{C}$ for $36 \mathrm{~h}$. We see additional peaks of iron oxide and body centered cubic (BCC) iron. The iron oxide peaks can be due to iron diffusing from the interface or the substrate up into the coating. This diffusion process leads to the formation of iron oxide. Authors in [11] report that diffusion of elements from the substrate can take place at high temperatures. In order to prevent this, a pre heat-treatment of SS in air at lower temperature can be carried out to form an oxide layer [11] that will act as a barrier for diffusion. The BCC iron peak that is present in XRD after $36 \mathrm{~h}$ of heat treatment was also present when only a SS substrate was heated to $650^{\circ} \mathrm{C}$ for $36 \mathrm{~h}$. This suggests that BCC iron peak occurs in the substrate due to heat treatment.

\subsection{Solar absorptance measurements}

The average solar absorptance measured for bulk tungsten sample was $\sim 48.4 \%$ and for laser sintered coating was $\sim 90.3 \%$.

a
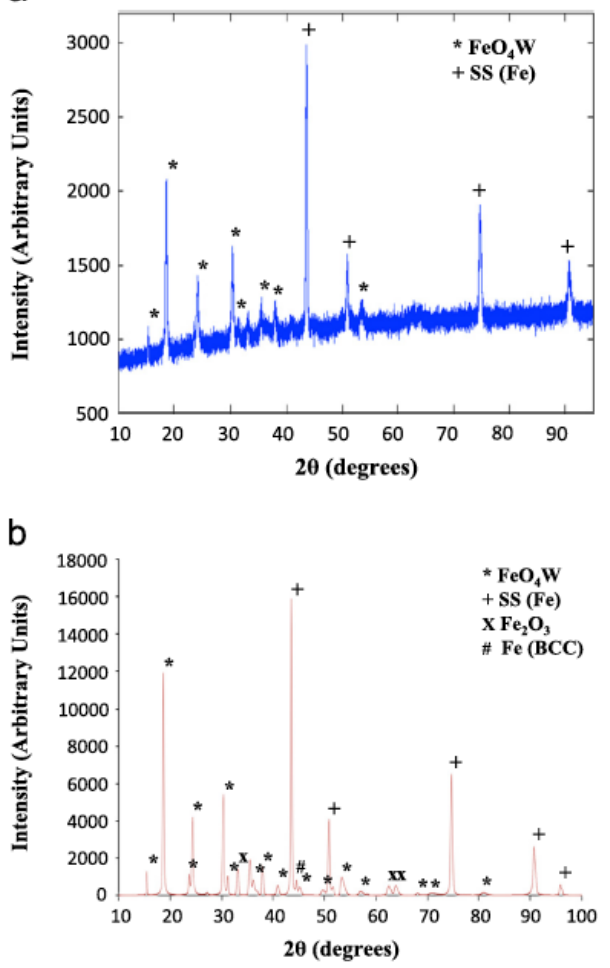

Fig. 3. XRD spectra of laser sintered coating (a) before and after heat treatment at $650^{\circ} \mathrm{C}$ for $2 \mathrm{~h}$ and (b) after heat treatment at $650^{\circ} \mathrm{C}$ for $36 \mathrm{~h}$. 
Thus having random roughness on a tungsten surface can drastically increase solar absorptance as needed for solar selective coatings. Solar light gets highly absorbed due to its interaction with surface roughness. The roughness, height, and spacing ranges from sub-micron to at least a couple of microns as can be seen from SEM images. The surface behaves as a graded index medium $[17,18]$ when the roughness is sub-wavelength thus leading to increased solar absorptance. When the surface roughness is longer than the wavelength, the increased absorptance is due to the light getting trapped by multiple reflections [14]. This light interaction with random roughness on the sintered layers lead to an overall high solar absorptance of $~ 90.3 \%$.

For high temperature performance, the sintered tungsten sample was heated at $650^{\circ} \mathrm{C}$ for $2 \mathrm{~h}, 12 \mathrm{~h}$, and then $36 \mathrm{~h}$ in air and after each heating cycle, its solar absorptance was measured. Table 1 below shows solar absorptance at room temperature and after heat treatment at $650{ }^{\circ} \mathrm{C}$. Fig. 4 shows a plot of absorptance of the coating before and after heat treatment in wavelength ranging from $410 \mathrm{~nm}$ to $3000 \mathrm{~nm}$.

To understand the slight change in solar absorptance and the role of oxidation, EDS study was conducted and the results are presented in Table 2.

The iron, chromium, and nickel peaks are due to elements present in stainless steel. The wt\% of iron has increased after heat treatment for $36 \mathrm{~h}$ and that could just be due to conducting EDS in a different region of the coating. Laser sintered coatings have surface oxide that was formed during atmospheric pressure sintering where high temperatures are generated. This surface oxide acts as a protection layer that prevents major oxidation of sintered coatings when being heated in air at $650{ }^{\circ} \mathrm{C}$. Authors in [19] present a similar observation where the optical properties of laser textured titanium surface remains unchanged before and after heat treatment in air at $500{ }^{\circ} \mathrm{C}$. Tungsten combines with oxygen to form tungsten oxide of various compositions. The oxide that has been

Table 1

Solar absorptance before and after heat treatment at $650^{\circ} \mathrm{C}$

\begin{tabular}{ll}
\hline & Solar absorptance $(\alpha)(\%)$ \\
\hline $\begin{array}{l}\text { Room } \\
\text { temperature }\end{array}$ & 90.3 \\
$650^{\circ} \mathrm{C}$ for $2 \mathrm{~h}$ & 87.0 \\
$650^{\circ} \mathrm{C}$ for $12 \mathrm{~h}$ & 87.2 \\
$650^{\circ} \mathrm{C}$ for $36 \mathrm{~h}$ & 87.0 \\
\hline
\end{tabular}

shown to be stable up to $800-900{ }^{\circ} \mathrm{C}$ in air is of type $\mathrm{WO}_{3}[20]$. Not only does the surface oxide serve as a protection layer, it can also serve as an index-matching layer at tungsten-air interface [21] that reduces front surface reflection, thereby contributing to higher solar absorptance. A slight increase in oxidation is seen after heat treatment of sintered coating that could have led to a small change in $\alpha$. It has been reported that oxidation of metallic nickel [22] and chromium [23] can lead to a slight decrease in solar absorptance. Both nickel and chromium are present in the sintered layer and their oxidation could have led $\alpha$ to change from $\sim 90 \%$ to $87 \%$. There is also a slight change in surface morphology where the periodicity of sub-micron surface roughness has decreased that could also cause the change in solar absorptance. It has been shown theoretically that pseudo-random structures on tungsten surface can achieve $\alpha>99 \%$ at room temperature [21] and here we have experimentally shown $\alpha \sim 90.3 \%$. Authors in [17] fabricated submicron cylindrical cavities in bulk tungsten and achieved $\alpha \sim 82 \%$ after annealing in vacuum at $1200 \mathrm{~K}$, however, no heat treatment in air was done. Authors in [24] fabricated dendritic textures on tungsten surface and were able to achieve $\alpha \sim 98.5$ after heat treatment in air only up to $275^{\circ} \mathrm{C}$ but a degradation in solar absorptance was observed at higher temperatures. To the best of our knowledge, this is the first experimental demonstration of achieving $\alpha \sim 87 \%$ after heat treatment in air at $650{ }^{\circ} \mathrm{C}$ for $36 \mathrm{~h}$ using tungsten.

\subsection{Infrared reflectance measurement}

Fig. 5 shows IR reflectance variation with wavelength for sintered coating before and after heat treatment. The shape of the curve before heat treatment is governed by surface morphology of the sintered tungsten. The peaks between 2.5 and $6 \mu \mathrm{m}$ before heat treatment is characteristic of the surface morphology of the coating. It has been shown [25] that surface gratings on tungsten surface produce similar peaks in the near infra-red region due to

Table 2

EDS data in weight (wt) percent before and after heat treatment at $650{ }^{\circ} \mathrm{C}$

\begin{tabular}{lccc}
\hline & Room temperature & $\mathbf{6 5 0}{ }^{\circ} \mathbf{C}$ for $\mathbf{2 ~ h}$ & $\mathbf{6 5 0}{ }^{\circ} \mathbf{C}$ for $\mathbf{3 6 ~ h}$ \\
\hline Tungsten & 28.42 & 27.95 & 21.40 \\
Iron & 38.66 & 35.78 & 42.30 \\
Chromium & 9.21 & 8.83 & 8.30 \\
Nickel & 3.19 & 2.74 & 2.40 \\
Oxygen & 20.52 & 24.70 & 25.60 \\
\hline
\end{tabular}

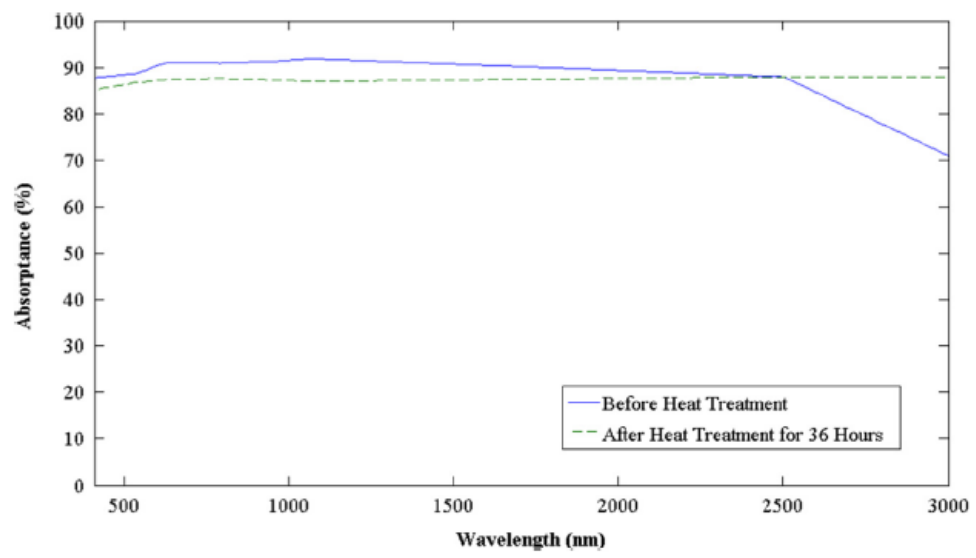

Fig. 4. Absorptance before and after heat treatment of laser sintered coating. 


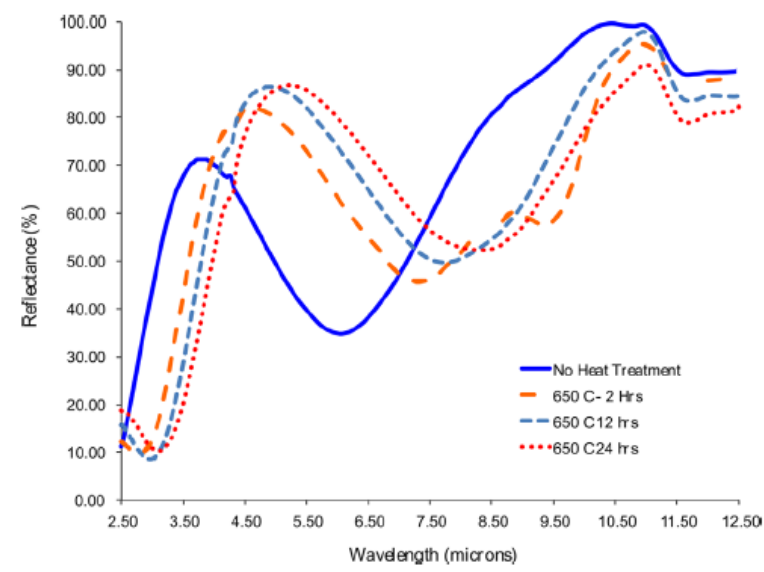

Fig. 5. IR reflectance of sintered coating (a) before heat treatment, (b) after heat treatment at $650{ }^{\circ} \mathrm{C}$ for $2 \mathrm{~h}$, (c) after heat treatment at $650^{\circ} \mathrm{C}$ for $12 \mathrm{~h}$, and (d) after heat treatment at $650^{\circ} \mathrm{C}$ for $36 \mathrm{~h}$.

plasmonic effects and this peak can shift based on the dimensions of the surface features. Beyond wavelength of $6 \mu \mathrm{m}$, the reflectance rises and approaches close to $90 \%$, as the wavelength is getting much larger than the surface roughness. There is a shift in the IR curve after heat treatment for $2 \mathrm{~h}$ but the overall shape is still similar. After that, there are only small shifts in the IR curve when the sintered coating is heat treated for $12 \mathrm{~h}$ and then for $36 \mathrm{~h}$.

\subsection{Simulation of IR reflectance}

To understand the shift in the IR peak from 3.7 to $4.7 \mu \mathrm{m}$ after heat treatment, theoretical simulation of reflectance of the sintered surface before and after heat treatment was performed. The simulation was carried out using finite difference time domain (FDTD) method [26] and the program was MIT electromagnetic equation propagation (MEEP) [27]. The FDTD method employs iterative solving of Maxwell's equations over a grid. Structures were described via the dielectric constants of the grid at each location. Perfectly matched layer (PML) boundary conditions were used in the direction of light propagation and Bloch periodic boundary conditions were used in the lateral direction. The reflectance spectra were obtained by subtracting the incoming wave from the reflected wave and the un-polarized light was approximated by averaging the reflectance spectra for $\mathrm{S}$ and $\mathrm{P}$ polarized light. The surface dimensions were obtained from the SEM images shown in Fig. 2b and c. Fig. 6 shows IR reflectance spectra of the simulation performed for sintered surface before heat treatment and after heat treatment for $2 \mathrm{~h}$. The slight change in periodicity of sub-micron surface roughness from $30 \mathrm{~nm}$ to $20 \mathrm{~nm}$ causes the shift in the peak. The sub-micron features on sintered surface before heat treatment have a larger periodicity and periodicity decreased after heat treatment since it gets a little smoother as seen in the SEM image. The SEM images are not that high in resolutions and so the program cannot approximate the exact surface. The purpose of the simulation is to explain the shift and not the magnitude of the reflectance values.

\subsection{Emittance and FOM}

From the IR reflectance, we can see that sintered coating maintains its performance after heat treatment and does not degrade, thus the protective surface oxide is crucial for maintaining performance of spectral selective coatings at high temperatures.

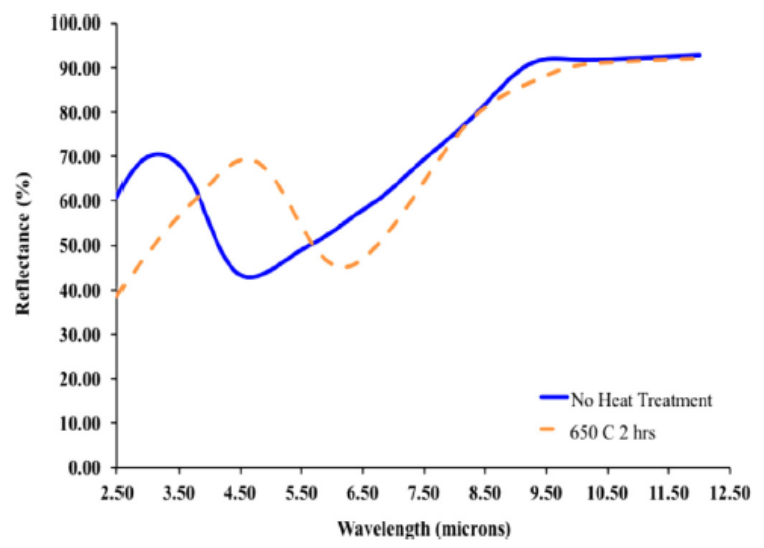

Fig. 6. Simulated IR reflectance of sintered coating before heat treatment and after heat treatment at $650^{\circ} \mathrm{C}$ for $2 \mathrm{~h}$.

Table 3

Emittance at room temperature and at $650{ }^{\circ} \mathrm{C}$.

\begin{tabular}{ll}
\hline & Emittance $(\varepsilon)(\%)$ \\
\hline Room temperature & 16.1 \\
$650^{\circ} \mathrm{C}$ for $2 \mathrm{~h}$ & 48.1 \\
$\mathbf{6 5 0} \mathrm{C}$ for $\mathbf{1 2} \mathrm{h}$ & 48.1 \\
$650^{\circ} \mathrm{C}$ for $36 \mathrm{~h}$ & 49.3 \\
\hline
\end{tabular}

Table 4

Selective absorber efficiency (\%).

\begin{tabular}{llll}
\hline \multirow{2}{*}{ Emittance } & \multicolumn{3}{l}{ Efficiency at various temperatures } \\
\cline { 2 - 4 } & $\mathbf{6 5 0}{ }^{\circ} \mathbf{C}$ & $\mathbf{7 0 0}{ }^{\circ} \mathbf{C}$ & $750{ }^{\circ} \mathbf{C}$ \\
\hline $\mathbf{0 . 4 9}$ & 83.6 & 82.9 & 81.9 \\
$\mathbf{0 . 7 0}$ & 82.2 & 81.1 & 79.8 \\
$\mathbf{0 . 9 0}$ & 80.8 & 79.4 & 77.7 \\
\hline
\end{tabular}

Emittance for the sintered coating is calculated from the IR reflectance plots in Fig. 5 using Eq. (1):

$\varepsilon(T)=\frac{\int_{0}^{\infty} E(T, \lambda) \times(1-R(\lambda)) d \lambda}{\int_{0}^{\infty} E(T, \lambda) d \lambda}$

where $E(T, \lambda)$ is blackbody irradiance at respective temperature, and $R(\lambda)$ is measured reflectance at room temperature from Fig. 5. Table 3 below shows the emittance of the laser sintered coating before and after heat treatment at $650{ }^{\circ} \mathrm{C}$. As mentioned earlier, the sintered coating maintains its performance fairly well after heat treatment for at least $36 \mathrm{~h}$. The emittance values after heat treatment are calculated using blackbody radiation at $650^{\circ} \mathrm{C}$. If the calculation was carried using blackbody radiation at room temperature, then the emittance of the coating after heat treatment would be $\sim 22.8 \%$ which is close to $16.1 \%$.

Table 4 below shows the selective absorber efficiency calculated using Eq. (2) that was provided by author in [28].

$\eta=\frac{\alpha C I-\varepsilon \sigma T^{4}}{C I}$

where $C$ is the concentration, $\sigma$ is the Stefan-Boltzmann constant $\left(5.67 \times 10^{-8} \mathrm{~W} / \mathrm{m}^{2} \mathrm{~K}^{-4}\right), T$ is the temperature $(\mathrm{K})$, and $I$ is the irradiance $\left(1000 \mathrm{~W} / \mathrm{m}^{2}\right)$. 
The solar concentration used was 600 and solar absorptance was 0.87 as achieved for laser sintered coating. Even though for high concentration applications, like in tower systems, maximizing solar absorptance is a primary concern, having low emittance can also provide improvements in receiver efficiency. We can see for current laser sintered coatings at $650^{\circ} \mathrm{C}$ that the selective absorber efficiency is $83.6 \%$ but if the emittance was at 0.9 , the efficiency would drop to $80.8 \%$. The concern becomes even greater at higher temperatures where the drop in efficiency is larger, for example at $750{ }^{\circ} \mathrm{C}$, the efficiency at emittance of 0.49 is $81.9 \%$ and for emittance of 0.9 is only $77.7 \%$. Thus the need to go to higher temperatures in the future will require emittance to be controlled for higher efficiencies.

The FOM was calculated using Eq. (1) for sintered coating heat treated at $650{ }^{\circ} \mathrm{C}$. Thermal emittance of laser sintered tungsten at $80^{\circ} \mathrm{C}$ was calculated by the formula given in [14] and emittance at $2400 \mathrm{~nm}$ was assumed to be equal to absorptance at $2400 \mathrm{~nm}$ according to Kirchoff's Law [1]. Thus, $\alpha_{\text {solar }} \sim 0.87, \varepsilon_{80} \mathrm{c} \sim 0.245$, and $\varepsilon_{2400 \mathrm{~nm}} \sim 0.81$ was used for sintered coating heated at $650^{\circ} \mathrm{C}$ for $36 \mathrm{~h}$. A high FOM of $\sim 50$ was achieved. In order to further increase the FOM, optimizing laser processing parameters and applying an anti-reflection (AR) coating would increase $\alpha_{\text {solar. }}$. It has been shown that applying coatings on textured metal surfaces [29] can improve solar absorptance and we have also done some preliminary work where we applied $\mathrm{TiO}_{x}$ coating on laser sintered nickel and gained an absolute $8 \%$ increase in solar absorptance. More details will be published in a separate paper. The application of AR coating will not only help with solar absorptance but might also help in achieving thermal stability in air allowing to reach temperatures beyond $650^{\circ} \mathrm{C}$.

\section{Conclusion}

Spectral selective coatings for solar thermal receivers have been fabricated by laser sintering of tungsten nano and micro particles on stainless steel substrate under atmospheric pressure that has high figure of merit. The performance of sintered tungsten remained relatively same before and after heat treatment up to $650{ }^{\circ} \mathrm{C}$ in air for at least $36 \mathrm{~h}$ due to the protective oxide layer that was formed on tungsten while laser sintering. The protective oxide prevented major oxidation of the sintered coating thus maintaining stability at high temperatures. Surface roughness of sintered coatings was optimized that ranged from nanoscale to $2 \mu \mathrm{m}$ that helped achieve spectral selectivity. A high solar absorptance, low thermal emittance, and in turn a high FOM of $\sim 50$ was achieved for laser sintered coating. The role of keeping emittance low, even for high concentration applications like tower systems, is emphasized by calculating selective absorber efficiency. This high FOM shows promise for laser sintered coatings to be used in solar tower receivers. A further increase in the performance can be expected by controlling the surface roughness through further optimization of laser processing parameters and by coating it with anti-reflection film.

\section{Acknowledgements}

We appreciate the financial support received through NASA Langley Professor Program. Thanks to Dr. Robert Moses at NASA Langley Research Center for his continued advice, encouragement, and support. We also thank Dr. Robert Bryant, Dr. Keith Gordon, Mr. James Baughman, Mr. Peter Messick, Mr. Harold Clay, Mr. David Hartman, and Mr. Joel Alexa, at NASA Langley Research Center for allowing access to IR measurement facility, SEM equipment, XRD equipment, and high temperature oven used in this research.

\section{References}

[1] A. Ambrosini, T. Lambert, M. Bencomo, A. Hall, K. Every, N. Siegel, C. Ho, Proceedings of the ASME 2011, Fifth International Conference on Energy Sustainability, Washington DC, 7-10 August 2011, pp. 1-8.

[2] A. Hall, A. Ambrosini, C. Ho, Solar selective coatings for concentrating solar power central receivers, Adv. Mater. Processes 170 (2012) 28-32.

[3] N. Selvakumar, H. Barshilia, Review of physical vapor deposited (PVD) spectrally selective coatings for mid- and high-temperature solar thermal applications, Sol. Energy Mater. Sol. Cells 98 (2012) 1-23.

[4] S. Esposito, A. Antonaia, M.L. Addonizio, S. Aprea, Fabrication and optimisation of highly efficient cermet-based spectrally selective coatings for high operating temperature, Thin Solid Films 517 (2009) 6000-6006.

[5] A. Berghaus, A. Djahanbakhsh, LK. Thomas, Characterisation of CVD-tungstenalumina cermets for high-temperature selective absorbers, Sol. Energy Mater Sol. Cells 54 (1998) 19-26.

[6] M. Farooq, M.G. Hutchins, Optical properties of higher and lower refractive index composites in solar selective coatings, Sol. Energy Mater. Sol. Cells 71 (2002) 73-83.

[7] D. Xinkang, W. Cong, W. Tianmin, Z Long, C. Buliang, R. Ning, Microstructure and spectral selectivity of $\mathrm{Mo}-\mathrm{Al}_{2} \mathrm{O}_{3}$ solar selective absorbing coatings after annealing, Thin Solid Films 516 (2008) 3971-3977.

[8] Q. Zhang, Y. Yin, D.R. Mills, High efficiency Mo- $\mathrm{Al}_{2} \mathrm{O}_{3}$ cermet selective surfaces for high-temperature application, Sol. Energy Mater. Sol. Cells 40 (1996) 43-53.

[9] Q. Zhang, Recent progress in high-temperature solar selective coatings, Sol. Energy Mater. Sol. Cells 62 (2000) 63-74.

[10] R.N. Schmidt, K.C. Park, High-temperature space-stable selective solar absorber coatings, Appl Opt 4 (1965) 917-925.

[11] F. Cao, K. McEnaney, G. Chen, Z Ren, A review of cermet-based spectrally selective solar absorbers, Energy Environ. Sci. 7 (2014) 1615-1627.

[12] J.-P. Kruth, G. Levy, F. Klocke, T.H.C. Childs, Consolidation phenomena in laser and powder-bed based layered manufacturing, CIRP Ann. Manuf. Technol. 56 (2007) $730-759$.

[13] P. Fischer, N. Karapatis, V. Romano, R Glardon, H.P. Weber, A model for the interaction of near-infrared laser pulses with metal powders in selective laser sintering, Appl. Phys. A 74 (2002) 467-474.

[14] A.A. Shah, M.C. Gupta, Spectral selective surfaces for concentrated solar power receivers by laser sintering of tungsten micro and nano particles, Sol. Energy Mater. Sol. Cells 117 (2013) 489-493.

[15] M.J. Persky, M. Szczesniak, Infrared, spectral, directional-hemispherical reflec tance of fused silica, Teflon polytetrafluoroethylene polymer, chrome oxide ceramic particle surface, Pyromark 2500 paint, Krylon 1602 paint, and Duraflect coating, Appl Opt 47 (2008) 1389-1396.

[16] L.C. Hsu, The stability relations of the wolframite series, Am. Mineral, 61 (1976) 944-955.

[17] H. Saia, H. Yugamia, Y. Kanamorib, K. Hane, Solar selective absorbers based on two-dimensional W surface gratings with submicron periods for hightemperature photothermal conversion, Sol. Energy Mater. Sol. Cells 79 (2003) 35-49.

[18] F. Ghmari, T, Ghbara, M. Laroche, R. Carminati, J.-J. Greffet, Influence of microroughness on emissivity, J. Appl. Phys. 96 (2004) 2656-2664.

[19] V. Iyengar, B. Nayak, M. Gupta, Ultralow reflectance metal surfaces by ultrafast laser texturing, Appl Opt 49 (2010) 5983-5988.

[20] N.M.G. Parreira, T. Polcar, A. Cavaleiro,Thermal, stability of reactive sputtered tungsten oxide coatings, Surf. Coat. Technol. 201 (2007) 7076-7082.

21] C. Ungaro, S. Gray, M. Gupta, Black tungsten for solar power generation, AppL. Phys. Lett. 103 (2013) 071105.

[22] A. Wazwaz, Accelerated heat degradation testing and characterization of nickel-pigmented aluminium oxide selective absorber, in: Second International Conference on the Palestinian Environment, 6 October 2009, pp. 38-46

[23] R. Pettit, C. Brinker, Use of sol-gel thin films in solar energy applications, Sol. Energy Mater. 14 (1986) 269-287.

[24] T. DiStefano, G. Pettit, J. Woodall, J. Cuomo, Conformal antireflective coatings on a textured tungsten surface, Appl. Phys. Lett. 32 (1978) 676-678.

[25] H. Sai, Y. Kanamori, K. Hane, H. Yugami, Numerical study on spectral properties of tungsten one-dimensional surface-relief gratings for spectrally selective devices, J. Opt. Soc. Am. A: Opt. Image Sci. Vis. 22 (2005) 1805-1813.

[26] A. Taflove, S. Hagness, Computational Electrodynamics: The Finite-Difference Time-Domain Method, third ed., Artech House, Norwood, MA, 2005.

[27] A. Oskooi, D. Roundy, M. Ibanescu, P. Bermel, J. Joannopoulos, S.G. Johnson, Meep: a flexible free-software package for electromagnetic simulations by the FDTD method, Comput. Phys. Commun. 181 (2010) 687-702.

[28] L. Cindrella, The real utility ranges of the solar selective coatings, Sol. Energy Mater. Sol. Cells 91 (2007) 1898-1901.

[29] N.P. Sergeant, M. Agrawal, P. Peumans, High performance solar-selective absorbers using coated sub-wavelength gratings, Opt. Express 18 (2010) $5525-5540$. 


\title{
Spectral selective surfaces for concentrated solar power receivers by laser sintering of tungsten micro and nano particles
}

\author{
Ankit A. Shah, Mool C. Gupta* \\ Department of Electrical and Computer Engineering University of Virginia, Charlottesville, Virginia 22904, USA
}

A R T I C L E I N F O

Artide history:

Received 13 March 2013

Received in revised form

19 June 2013

Accepted 12 July 2013

Keywords:

Spectral selective surface

Solar thermal

Concentrating sola

Laser sintering

Sub-micron surface roughness

Tungsten nano particles

\begin{abstract}
A B S T R A C T
Spectral selective surfaces for concentrated solar power receivers have been fabricated by laser sintering of tungsten micro and nano particles on stainless steel substrate under atmospheric pressure. Solar absorptance of $\sim 83 \%$ has been experimentally measured for laser sintered tungsten layer and thermal emittance of $\sim 11.6 \%$ at $300 \mathrm{~K}$ was computed based on the IR reflectance measurement. This high solar absorptance and low thermal emittance was achieved by a one micron thick laser sintered layer having sub-micron surface roughness with random spacing in the range from nanoscale to $5 \mu \mathrm{m}$. Laser sintering has not been applied for fabrication of solar receiver coatings and the first experimental demonstration is presented in this paper. Laser sintering under atmospheric pressure is a cost effective method for solar thermal applications.
\end{abstract}

(c) 2013 Elsevier B.V. All rights reserved.

\section{Introduction}

Concentrating solar power (CSP) or solar thermal systems is a growing sector as an alternative energy source. Besides consumer applications, solar thermal energy also has tremendous potential for space applications. NASA and other space agencies have researched into utilizing solar thermal energy for rocket propulsion applications due to higher performance than chemical propulsion. CSP is not only limited to conventional rockets, but can also provide propulsion and on-board power for mini and micro satellites in space. For a higher overall conversion efficiency, solar power receivers need to have high photothermal conversion at very high operating temperatures of $>500{ }^{\circ} \mathrm{C}$. In order to achieve high photothermal conversion efficiency, the receiver needs to have high solar absorptance $(\alpha) \geq 95 \%$, and low thermal emittance $(\varepsilon) \leq 10 \%$ at high operating temperature. For these surfaces to have high $\alpha$ in the solar spectral region, for wavelengths $(\lambda) \leq 2 \mu \mathrm{m}$, and low $\varepsilon$ in the infra-red (IR) region, for $\lambda \geq 2 \mu \mathrm{m}$, they need to be spectrally selective.

Currently, commercial spectral selective coatings for receivers show good performance and stability up to $500^{\circ} \mathrm{C}$ in vacuum but performance degrades due to oxidation [1]. Current manufacturing techniques with $\alpha>90 \%$, and $\varepsilon<10 \%$ involve vacuum fabrication techniques making the process expensive.

\footnotetext{
* Corresponding author. Tel.: +1 7573256850.

E-mail address: mgupta@virginia.edu (M.C. Gupta).
}

Materials that have intrinsic optical properties to provide high spectral selectivity, i.e. $\alpha>95 \%$ and $\varepsilon<10 \%$ at temperatures higher than $500{ }^{\circ} \mathrm{C}$, are not found in nature. Metals like gold, silver, etc. have low thermal emittance but also a very low solar absorptance. Transparent materials like oxides, nitrides, and carbides have extremely low absorptance and very high emittance. There have been previous attempts by researchers to use semiconductors like silicon ( $\mathrm{Si}$ ) and germanium (Ge) as absorber surfaces [2]. Si and $\mathrm{Ge}$ have relatively high solar reflectance and thus broadband antireflection coatings need to be used to increase $\alpha$. However, even with the antireflection coatings, the IR reflectance is low which contributes to high $\varepsilon$. Also, the performance degrades at higher temperatures due to thermal oxidation. Thus a combination of different materials is needed to be used as receiver surfaces for CSP.

Multilayers of dielectric and metal films are stacked to form the absorber surface. These layers achieve spectral selectivity by utilizing partial absorptance in metal films, interference due to multiple layers [3], and IR reflectivity from metals. Several multilayer absorbers using different metals (e.g., $\mathrm{Mo}, \mathrm{Ag}, \mathrm{Cu}$, and $\mathrm{Ni}$ ) and dielectric layers (e.g., $\mathrm{Al}_{2} \mathrm{O}_{3}, \mathrm{SiO}_{2}, \mathrm{CeO}_{2}$, and $\mathrm{ZnS}$ ) have been investigated for CSP applications [2]. The inter-diffusion between layers at higher operating temperatures causes performance degradation. The manufacturing of these layers requires a vacuum environment and high cost equipment to control the precise thickness of the films which makes it an expensive process.

Ceramic-metal composite (cermet) is a mixture of metallic particles in a dielectric host. Cermet layers deposited onto a 
metallic film have been used for spectral selectivity. The cermet layers act as absorbers in the solar region [2] and the underlying metal layer acts as a reflector for IR to keep the emittance low. Cermet layers serve as a graded index material due to which the reflection of solar spectrum is reduced and the absorptance is increased due to electromagnetic (EM) wave interaction with metal particles and interference phenomenon. The multilayer structure of cermets and metal layer has been fabricated and characterized [4-10] and can achieve relatively high solar absorptance and low thermal emittance. However, performance degrades when these layers are exposed to air due to oxidation at higher temperatures. In addition, like other methods, the manufacturing cost of these cermet layers is extremely high due to the need of vacuum fabrication techniques.

Surface texturing of materials has been applied for solar energy applications [2]. Texturing Si is a good way to significantly increase solar absorptance for solar cell applications. For CSP, cermets and metals have been textured to achieve solar selectivity. Optical trapping of solar light through multiple reflections results in high solar absorptance. Usually, it is easier to achieve high solar absorptance but keeping emittance low is a challenge [11-15]. Also, the performance of textured surfaces drops due to oxidation at high temperatures. There have been some attempts of texturing materials in air environment [16] but have yet to show promising results. There is a large need to overcome the limitations of previous methods and develop highly efficient spectral selective surfaces at relatively lower manufacturing cost that can operate to temperatures above $500{ }^{\circ} \mathrm{C}$

In this paper, we report a new approach to fabricate spectral selective surfaces using pulsed laser sintering of tungsten metallic micro and nano particles under atmospheric pressure. Laser sintering has been used before for fabrication of coatings using high temperature materials $[17,18]$ but it has not been investigated for fabrication of spectral selective surfaces required in CSP systems.

Controlling the surface roughness of the sintered layer is the key to achieving spectral selectivity. Previously, sub-wavelength periodical tungsten $(\mathrm{W})$ structures have been fabricated by fast atom beam etching to have $\alpha \sim 82 \%$ and $\varepsilon \sim 5.6 \%$ at $400 \mathrm{~K}$ and $15.9 \%$ $1200 \mathrm{~K}$ [13]. The fabricated sub-micron holes on tungsten can cause standing wave resonances that have been attributed to increase broad wavelength absorptance [13]. However, this required expensive tungsten substrate and complex fabrication process. Sub-wavelength structures on metal surfaces can increase solar absorptance due to surface plasmon absorption $[19,20]$ and also due to the surface behaving like graded index medium thus providing antireflection $[13,19]$. IR reflectance from subwavelength structures on metal surface can be kept very high because the IR wavelengths are much longer than the dimensions of surface roughness and therefore the surface appears smooth and radiates as a flat surface [14]. In this study we show that random sub-wavelength structures can be obtained through laser sintering process at atmospheric pressure, allowing fabrication of low cost high efficiency receivers for solar thermal applications.

\section{Experiment}

During pulsed laser sintering, a beam is focused onto metallic particles and light gets absorbed thus raising the temperature of the particles. If the temperature is high then the surface of the particles melt and molten particles form necks adjacent to each other forming a continuous layer. For large area sintering, the sample is scanned under the laser beam using a computer controlled $\mathrm{X}-\mathrm{Y}$ stage. Fig. 1 below shows the laser sintering concept.

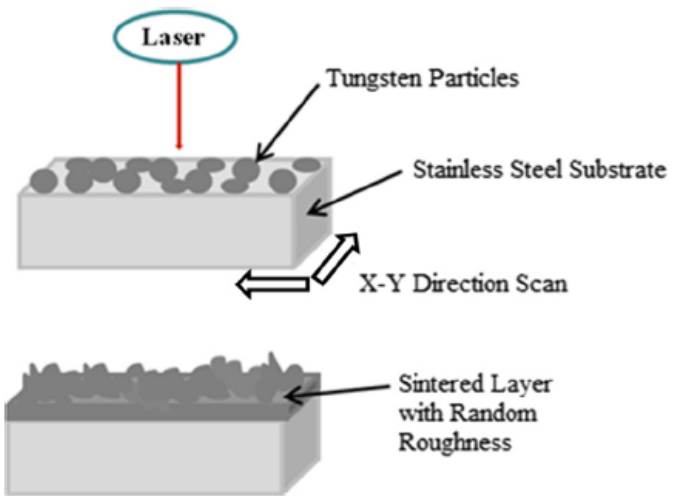

Fig. 1. Laser is scanned across the substrate and it irradiates and melts the metallic particles causing them to bind together to form a sintered layer.

Laser sintering of $\mathrm{W}$ micro $(1-5 \mu \mathrm{m})$ and nano $(80-100 \mathrm{~nm})$ particles, supplied by SkySpring Nanomaterials Inc. was carried out on stainless steel (SS) substrate to form a spectral selective layer. Tungsten was chosen as a material of choice because it intrinsically has relatively high absorptance in the solar spectrum and low emittance in the IR. The tungsten powder was put in a beaker containing isopropyl alcohol and the mixture was then ultrasonicated for $5 \mathrm{~min}$. The ultrasonication was done to break the agglomeration of tungsten nanoparticles. A dropper was then used to deposit the solution mixture from the beaker onto a SS substrate and it was kept aside at room temperature for the alcohol to evaporate. After approximately $20 \mathrm{~min}$, the alcohol evaporated leaving only W powder on SS substrate, which we refer to as sample. This sample was then mounted on a computer controlled X-Y stage for laser sintering. A $1064 \mathrm{~nm}$ wavelength laser with a repetition rate of $50 \mathrm{kHz}$ and a lens with focal length of $40 \mathrm{~mm}$ were used for laser sintering. The laser energy density used for sintering varied from 0.8 to $1.3 \mathrm{~J} / \mathrm{cm}^{2}$. The laser beam was incident on the sample as it was scanned in horizontal and vertical directions resulting in sintered $\mathrm{W}$ layer on SS. The scan rate of the $\mathrm{X}-\mathrm{Y}$ stage was $1 \mathrm{~mm} / \mathrm{s}$. After this first laser scan, tungsten powder was again deposited on the sintered region and the same procedures as listed above were followed for the second scan. Laser sintering was carried out under atmospheric pressure. Solar absorptance measurement was done using an integrating sphere, supplied by Labsphere Inc., and laser wavelengths of $532 \mathrm{~nm}$, $633 \mathrm{~nm}, 1064 \mathrm{~nm}$, and $1550 \mathrm{~nm}$. Reflectance measurements in the IR were done using a continuum IR microscope that was purchased from Thermo Fisher Scientific Inc.. The sample was illuminated by IR wavelengths ranging from 4 to $12 \mu \mathrm{m}$ and then reflected power was measured to provide a reflectance versus wavelength curve. The IR microscope has the ability to collect reflected light at an angular range of $+/-35^{\circ}$ from normal to the sample. Emittance was calculated based on experimental reflectance data and blackbody irradiance spectrum at $300 \mathrm{~K}$.

\section{Results and discussion}

3.1. Morphological study of laser sintered W micro and nano particles

Fig. 2a shows a scanning electron microscope (SEM) image of raw tungsten powder. Most particles are sub-micron $(80-200 \mathrm{~nm})$ in size but some of them are in the rage of $1-5 \mu \mathrm{m}$. After laser sintering, a sintered layer of $\mathrm{W}$ is formed as shown in Fig. $2 \mathrm{~b}$. The layer is continuous without any cracks or discontinuity. Fig. $2 c$ 

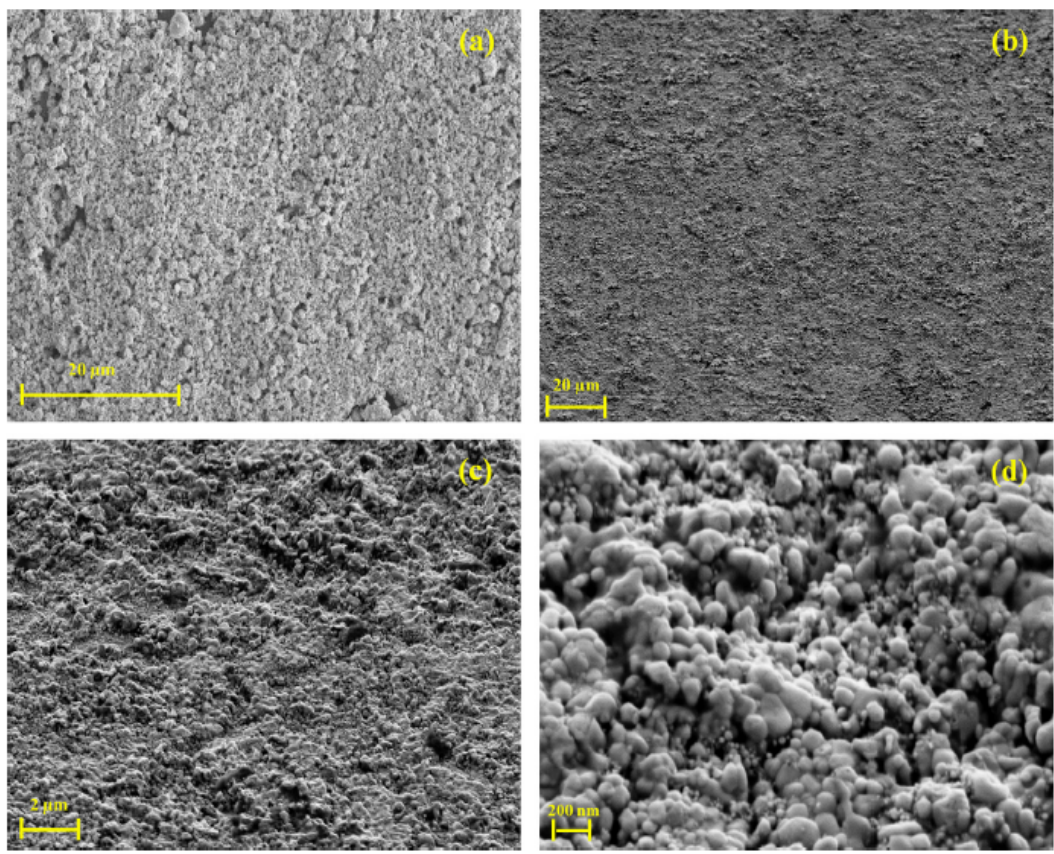

Fig. 2. SEM images of (a) loose tungsten powder, (b) sintered tungsten layer, (c) sintered tungsten layer at higher magnification, and (d) sintered tungsten layer at highest magnification.

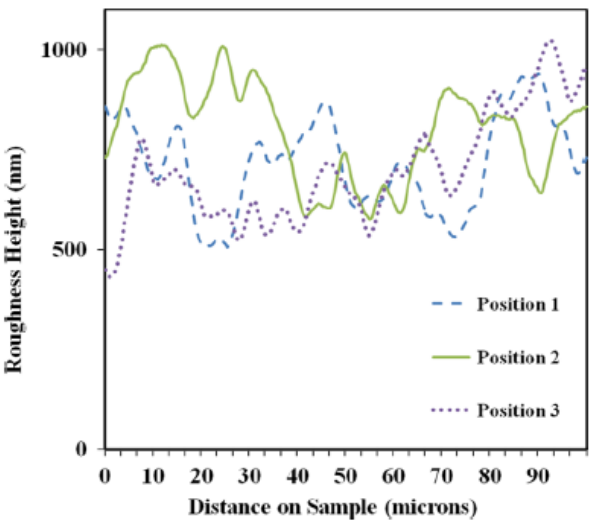

Fig. 3. Surface profilometer data taken at different positions across a distance of $100 \mu \mathrm{m}$ on sintered W layer.

shows a higher magnification image of the sintered $\mathrm{W}$ layer. The film is continuous and the roughness is starting to become more apparent in the image. The topography of the surface is not uniform which is in fact desired because the surface roughness of the sintered layer is used to achieve spectral selectivity. The roughness has random spacing on a micron and nanoscale level as seen in Fig. 2c and d.

Surface profilometer measurements were done on the sintered layer to examine the variation in roughness across the sample. Fig. 3 shows roughness height variation taken at different positions on the sample across a distance of $100 \mu \mathrm{m}$. The surface roughness height mostly lies in the range of $490-1010 \mathrm{~nm}$. The height variation between two nearest point that can be analyzed by the profilometer is around one micron. Thus submicron variations in height are not seen in the profilometer but can be seen in the SEM in Fig. $2 \mathrm{c}$ and d. Both roughness height and spacing contribute to spectral selectivity and therefore SEM and profilometer data have to be used in conjunction for spectral selectivity investigation.

\subsection{Absorptance}

The average solar absorptance of our sintered W layer was measured as $\sim 83 \%$. Polished tungsten has solar absorptance of $\sim 52 \%$. Thus it can be seen that having random roughness on tungsten surface can drastically increase solar absorptance. This increased absorptance is due to the fact that solar wavelengths interact with surface roughness ranging from nanoscale to microscale. The roughness height and spacing ranges from a few tens of nanometers to $5 \mu \mathrm{m}$ as can be seen from SEM and profilometer data. When the roughness is sub-wavelength, the increased solar absorptance can be explained by the effect of the surface behaving as a graded index medium thus providing antireflection $[13,19]$. When the surface roughness is longer than the wavelength, the increased absorptance is due to the EM wave getting trapped by multiple reflections. The remaining energy that is not absorbed is collected by the integrating sphere and thus all of the scattered energy is measured. The combined contribution from the above mentioned effects leads to an overall higher solar absorptance, $\sim 83 \%$, for the sintered layer.

\subsection{Reflectance and emittance}

Fig. 4 shows experimentally measured curve for reflectance versus IR wavelength for polished gold, polished stainless steel, polished tungsten, and laser sintered tungsten. Reflectance of gold and tungsten is $\sim 99 \%$ and $98 \%$ respectively at $4 \mu \mathrm{m}$ wavelength and beyond. These data are close to theoretical reflectance values in the IR spectrum that can be calculated using refractive index data [21]. The reflectance of polished stainless steel is also shown as it is the substrate on which laser sintered tungsten layer is fabricated. The ideal case is to have IR reflectance of the sintered 


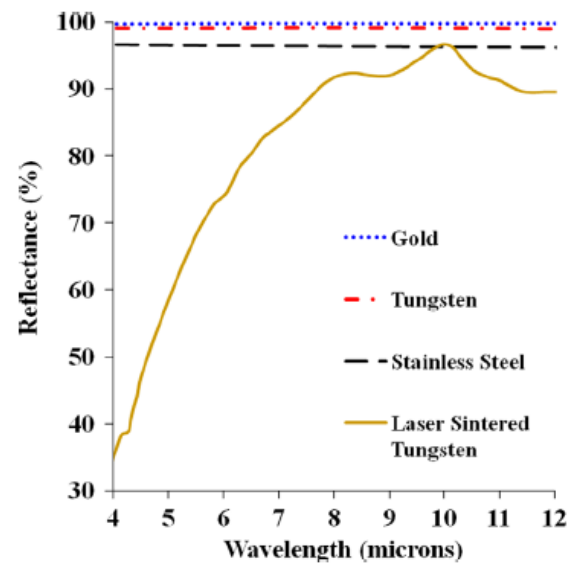

Fig. 4. Measured reflectance for gold, stainless steel, tungsten, and laser sintered tungsten.

tungsten layer close to polished tungsten so that the emittance will be low. As seen from SEM (Fig. 2b-d) and profilometer data (Fig. 3), the roughness is on the range from few tens of nanometers to $5 \mu \mathrm{m}$. Therefore, the IR radiation interacts with surface roughness. In Fig. 4, the reflectance of sintered tungsten is $\sim 35-75 \%$ in the wavelength range of $4-6 \mu \mathrm{m}$ as compared to $\sim 98 \%$ for polished tungsten. As mentioned earlier, if the wavelength is larger than the roughness, enhanced absorption would be expected due to the material behaving as a graded index medium. However, as the IR wavelength increases, the IR reflectance also increases. The reason is that the roughness is then much smaller than the wavelength and therefore the reflectance starts to rise and approach the value of polished tungsten as seen in Fig. 4. The intrinsic reflectance of tungsten metal is much higher in the IR than in the visible spectrum. This helps to achieve higher reflectance values in the IR. During IR reflectance measurements, most of the IR scattered light gets captured by the equipment as it can collect in the range of $+1-35^{\circ}$ from the normal. Some small amount of scattered energy is lost which would indicate as being absorbed by the surface and thus resulting in a slightly lesser value in reflectance. The equipment was capable of measuring reflectance only up to $12 \mu \mathrm{m}$. To account for reflectance beyond $12 \mu \mathrm{m}$, it is safe to assume that reflectance remains constant. This assumption is based on the fact that tungsten, theoretically, maintains a constant reflectance value of $\sim 98.3 \%$ up to $24 \mu \mathrm{m}$ in the IR spectrum [22]. Also, since the wavelength beyond $12 \mu \mathrm{m}$ is much larger than the sintered roughness, the reflectance for these higher wavelengths will be fairly constant. Using this measured reflectance data and extrapolating to higher wavelengths as mentioned above, emittance at $300 \mathrm{~K}$ was calculated for sintered tungsten layer. Thermal emittance was calculated by the formula given below

$\varepsilon(T)=\frac{\int_{0}^{\infty} E(T, \lambda)(1-R(\lambda)) d \lambda}{\int_{0}^{\infty} E(T, \lambda) d \lambda}$

where $E(T, \lambda)$ is blackbody irradiance at temperature $T$ and $R(\lambda)$ is measured reflectance. Thermal emittance of laser sintered tungsten was calculated as $\sim 11.6 \%$ at $300 \mathrm{~K}$. In order to further lower the emittance, laser parameters will be optimized so that resulting maximum roughness size is no larger than $1 \mu \mathrm{m}$.

\subsection{Cost reduction by the laser sintering process}

To provide a justification of cost benefit for the laser sintering process as compared to cermets that need a vacuum process, we use Schott Solar PTR 70 [23] receiver for parabolic trough plants as an example. The cost to buy a vacuum receiver is typically $€ 200-$ $€ 300$ per meter [24]. The receiver is $\sim 4 \mathrm{~m}$ in length, thus the cost of each receiver at current foreign exchange rate of $€ 1-\$ 1.30$ can be expected to be $\sim \$ 1043-\$ 1565$.

For our laser sintering process we use tungsten nanoparticles purchased from SkySpring Nanomaterials Inc. which cost \$1867 for $1 \mathrm{~kg}$. The length and outer diameter of the PTR 70 receiver are $4.060 \mathrm{~m}$ and $0.070 \mathrm{~m}$ respectively. The thickness of our sintered coating is approximately one micron. The density of tungsten is $19.25 \mathrm{~g} / \mathrm{cm}^{3}$. Therefore, approximately $17.19 \mathrm{~g}$ of material is needed to coat the PTR 70 receiver. Thus, the cost of $\mathrm{W}$ material per tube is approximately $\$ 32$. If we assume that the cost of purchasing nanoparticles will be similar to the cost of microparticles ( $\$ 143 / \mathrm{kg}$ by Inframat Advanced Materials) in the near future, then the cost of $W$ needed per tube will be $\sim \$ 2.50$.

Next we calculate process cost of laser sintering. High power fiber lasers $\sim 500 \mathrm{~W}$ are expected to cost $\sim \$ 95,000$ (IPG Photonics Corporation) that have a life time of $100,000 \mathrm{~h}$. If five of these lasers are used in conjunction, the cost incurred for laser use (electricity cost not included) $\sim \$ 4.75 / \mathrm{hr}$. The time needed to sinter one layer for the area of PTR 70 receiver will be $4 \mathrm{~h}$. And since two layers of sintering are needed, the total time needed to sinter will be $8 \mathrm{~h}$ per tube. Hence, the process cost per tube comes to $\$ 38$. Therefore, the total cost per tube, raw material needed plus laser uses, comes to $\sim \$ 40$. This shows that the manufacturing cost of laser sintering is a small fraction of the selling price of PTR 70 receiver and hence laser processing can be a cost effective method. Optimization of laser process can further reduce this cost.

\section{Conclusion}

A spectrally selective surface has been fabricated by laser sintering of tungsten micro and nano particles on stainless steel substrates under atmospheric pressure. This is the first successful experimental demonstration of laser sintering of micro/nano tungsten particles for fabrication of coatings for concentrated solar receivers. The laser sintering process allows controlling the surface roughness of sintered layer ranging from nanoscale to $5 \mu \mathrm{m}$. Solar absorptance of $\sim 83 \%$ has been experimentally measured for laser sintered tungsten layer and thermal emittance of $\sim 11.6 \%$ at $300 \mathrm{~K}$ was computed based on the IR reflectance measurement. Surface roughness of laser sintered tungsten is used to achieve spectral selectivity. The surface roughness traps EM waves by multiple reflections and through graded index medium since the interacting wavelengths are both smaller and larger than roughness dimensions. The further reduction of thermal emittance can be expected by controlling the surface roughness to less than $1 \mu \mathrm{m}$.

\section{Acknowledgments}

We appreciate the financial support received through NASA Langley Professor Program. Thanks to Dr. Robert Moses at NASA Langley Research Center for his continued advice, encouragement, and support. We also thank Dr. Robert Bryant, Dr. Keith Gordon, and Mr. James Baughman at NASA Langley Research Center for allowing access to IR measurement and SEM equipment used in this research.

\section{References}

[1] N. Selvakumar, H. Barshilia, Review of physical vapor deposited (PVD) spectrally selective coatings for mid- and high-temperature solar thermal applications, Solar Energy Materials and Solar Cells 98 (2012) 1-23. 
[2] C.E. Kennedy, Review of Mid- to High-Temperature Solar Selective Absorber Materials, NREL/TP-520-31267, National Renewable Energy LaboratoryGolden, CO, 2002

[3] X. Li, Y. Chen, J. Miao, P. Zhou, Y. Zheng, L. Chen, High solar absorption of a multilayered thin film structure, Optics Express 15 (2007) 1907-1912.

[4] S. Esposito, A. Antonaia, M.L. Addonizio, S. Aprea, Fabrication and optimisation of highly efficient cermet-based spectrally selective coatings for high operating temperature, Thin Solid Films 517 (2009) 6000-6006.

[5] A. Berghaus, A. Djahanbakhsh, L.K. Thomas, Characterisation of CVD-tungstenalumina cermets for high-temperature selective absorbers, Solar Energy Materials and Solar Cells 54 (1998) 19-26.

[6] M. Farooq, M.G. Hutchins, Optical properties of higher and lower refractive index composites in solar selective coatings, Solar Energy Materials and Sola Cells 71 (2002) 73-83.

[7] D. Xinkang, W. Cong, W. Tianmin, Z. Long, C. Buliang, R. Ning, Microstructure and spectral selectivity of $\mathrm{Mo}-\mathrm{Al}_{2} \mathrm{O}_{3}$ solar selective absorbing coatings after annealing, Thin Solid Films 516 (2008) 3971-3977.

[8] Q. Zhang, Y. Yin, D.R. Mills, High efficiency Mo- $\mathrm{Al}_{2} \mathrm{O}_{3}$ cermet selective surfaces forhigh-temperature application, Solar Energy Materials and Solar Cells 40 (1996) 43-53.

[9] Q. Zhang, Recent progress in high-temperature solar selective coatings, Solar Energy Materials and Solar Cells 62 (2000) 63-74.

[10] R.N. Schmidt, K.C. Park, High-temperature space-stable selective solar absorber coatings, Applied Optics 4 (1965) 917-925.

[11] A.Y. Vorobyev, G. Chunlei, Proceedings of the 2010 International Conference on Biosciences (BIOSCIENCESWORLD '10), Cancun, 7-13 March, 2010, pp, 135-138.

[12] C.M. Lampert, Coatings for enhanced photothermal energy collection I. Selective absorbers, Solar Energy Materials 1 (1979) 319-341.

[13] H. Saia, H. Yugamia, Y. Kanamorib, K. Hane, Solar selective absorbers based on two-dimensional $W$ surface gratings with submicron periods for high- temperature photothermal conversion, Solar Energy Materials and Solar Cells 79 (2003) 35-49.

[14] A. Lasagni, M. Nejati, R. Clasen, F. Mücklich, Periodical surface structuring of metals by laser interference metallurgy as a new fabrication method of textured solar selective absorbers, Advanced Engineering Materials 8 (2006) $580-584$.

[15] P.M. Curmi, G.L. Harding, Surface texturing of copper by sputter etching with applications for solar selective absorbing surfaces, Journal of Vacuum Science and Technology 17 (1980) 1320-1325.

[16] T. Hwang, A.Y. Vorobyev, C. Guo, Formation of solar absorber surface on nickel with femtosecond laser irradiation, Journal of Applied Physics A 108 (2012) 299-303.

[17] C Sun, Laser sintering of ultra high temperature materials, University of Virginia, Charlottesville, Virginia, 2010. (Ph.D. thesis).

[18] C. Sun, M.C. Gupta, E.A. Payzant, Effect of laser sintering on $\mathrm{Ti}-\mathrm{ZrB}_{2}$ mixtures, Journal of the American Ceramic Society 94 (2011) 3282-3285.

[19] F. Ghmari, T. Ghbara, M. Laroche, R. Carminati, J.-J. Greffet, Influence of microroughness on emissivity, Journal of Applied Physics 96 (2004) 2656-2664.

[20] S. Lal, S. Link, N.J. Halas, Nano-optics from sensing to waveguiding, Nature Photonics 1 (2007) 641-648.

[21] E.D. Palik, Handbook of Optical Constants of Solids, Academic Press, New York, 1985.

[22] 〈http://refractiveindex.info/?group=METALS\&material=Tungsten〉, June 5, 2013.

[23] 〈http://www.us.schott.com/csp/english/schott-solar-ptr-70-receivers.html).

[24] Ernst \& Young and Fraunhofer Institute, MENA Assessment of the Local Manufacturing Potential for Concentrated Solar Thermal Power (CSP) Projects. http://arabworld.worldbank.org/content/awi/en/home/research/mena_solar. html/, 2011 (accessed 5.06.13). 